\title{
The Complexity of Computational Problems about Nash Equilibria in Symmetric Win-Lose Games*
}

\author{
Vittorio Bilò $^{\dagger} \quad$ Marios Mavronicolas ${ }^{\ddagger}$
}

(JULY 25, 2019)

\begin{abstract}
We revisit the complexity of deciding, given a bimatrix game, whether it has a Nash equilibrium with certain natural properties; such decision problems were early known to be $\mathcal{N} \mathcal{P}$-hard [20. We show that $\mathcal{N} \mathcal{P}$-hardness still holds under two significant restrictions in simultaneity: the game is win-lose (that is, all utilities are 0 or 1 ) and symmetric. To address the former restriction, we design win-lose gadgets and a win-lose reduction; to accomodate the latter restriction, we employ and analyze the classical GHR-symmetrization 21] in the win-lose setting. Thus, symmetric win-lose bimatrix games are as complex as general bimatrix games with respect to such decision problems.

As a byproduct of our techniques, we derive hardness results for search, counting and parity problems about Nash equilibria in symmetric win-lose bimatrix games.
\end{abstract}

*This work was partially supported by the Italian Ministry of Education, Universities and Research (PRIN 2010-2011 research project ARS TechnoMedia: "Algorithmics for Social Technological Networks"), and by research funds at the University of Cyprus. This work extends and improves results that appeared in preliminary form in the authors' paper "The Complexity of Decision Problems about Nash Equilibria in Win-Lose Games," Proceedings of the 5th International Symposium on Algorithmic Game Theory, pp. 37-48, Vol. 7615, Lecture Notes in Computer Science, Springer-Verlag, October 2012.

${ }^{\dagger}$ Department of Mathematics and Physics "Ennio De Giorgi", University of Salento, 73100 Lecce, Italy. Part of the work of this author was done while visiting the Department of Computer Science, University of Cyprus. Email vittorio.bilo@unisalento.it

${ }^{\ddagger}$ Department of Computer Science, University of Cyprus, Nicosia CY-1678, Cyprus. Email mavronic@cs.ucy.ac.cy 


\section{Introduction}

\subsection{Framework and Motivation}

\subsubsection{Nash Equilibria, Win-Lose Bimatrix Games and Symmetric Games}

Among the most fundamental computational problems in Algorithmic Game Theory are those concerning the Nash equilibria [29, 30] of a game, where no player could unilaterally deviate to increase her expected utility. Such problems have been studied extensively [1, 4, 7, 9, 10, 11, 12, 13, 14, 20, 25, 26] for 2-player games with rational utilities given by a bimatrix. There are two prominent special cases of such general bimatrix games: win-lose and symmetric.

- Utilities are taken from $\{0,1\}$ in win-lose bimatrix games, originally put forward in [12].

- In a symmetric game [29, 30], players have identical strategy sets and the utility of a player is determined by the multiset of strategies chosen by her and the other players, with no discrimination. By a classical result of Nash, every symmetric game has a symmetric Nash equilibrium, where all players are playing the same mixed strategy [29, 30]. A symmetrization transforms a given bimatrix game into a symmetric one; the target is that a Nash equilibrium for the original bimatrix game can be reconstructed efficiently from a (symmetric) Nash equilibrium for the symmetric bimatrix game (cf. [8, 17, 21]).

\subsubsection{The Search Problem}

The fundamental theorem of Nash [29, 30] that a Nash equilibrium is guaranteed to exist for a finite game makes its search problem total; hence, the search problem is not $\mathcal{N} \mathcal{P}$-hard unless $\mathcal{N P}=$ co- $\mathcal{N P}$ [27, Theorem 2.1]. In a series of breakthrough papers culminating in [9, 14], it was established that, even for bimatrix games, the search problem is complete for $\mathcal{P} \mathcal{P} \mathcal{A D}$ [31], a complexity class capturing the computation of discrete fixed points; under suitable formulations, the problem is $\mathcal{F} \mathcal{I} \mathcal{X} \mathcal{P}$-complete for games with more than two players [16, Theorem 18].

Abbott, Kane and Valiant [1] presented a polynomial time transformation of a general bimatrix game into a win-lose bimatrix game, accompanied with a polynomial time map returning a Nash equilibrium for the general game when given one for the win-lose game. So the search problem is $\mathcal{P} \mathcal{P} \mathcal{A D}$-hard for win-lose bimatrix games, which suggests that hardness is not due to the rational utilities. The search problem for a symmetric Nash equilibrium in a symmetric bimatrix game is also $\mathcal{P} \mathcal{P} \mathcal{A} \mathcal{D}$-hard, thanks to the two symmetrizations from 1950 due to Brown and von Neumann [8] and due to Gale, Kuhn and Tucker [17, respectively; the complexity of the search problem for any Nash equilibrium in a symmetric bimatrix game has been mentioned as an open problem by Papadimitriou [32, Section 2.3.1]. 


\subsubsection{Decision, Counting and Parity Problems}

Decision problems arise naturally by twisting the search problem in simple ways that deprive it from its existence guarantee for a Nash equilibrium. Here is a non-exhaustive list of such natural decision problems (see Section 2.3 for formal statements): given a game, does it have:

(i) At least $k+1$ Nash equilibria for some fixed integer $k \geq 1$ ? (The special case with $k=1$ was introduced in [20]; the cases with $k>1$ are considered here for the first time.)

(ii) A Nash equilibrium where each player has expected utility at least (resp., at most) a given number? 20]

(iii) A Nash equilibrium where the total expected utility of players is at least (resp., at most) a given number? [13]

(iv) A Nash equilibrium where the players' supports contain (resp., are contained in) a given set of strategies? [20]

(v) A Nash equilibrium where the players' supports have sizes greater (resp., smaller) than a given integer? [20]

(vi) A Nash equilibrium where each player is using uniform probabilities on her support? [7]

(vii) A Nash equilibrium where some player is using non-uniform probabilities on her support?

(viii) A symmetric Nash equilibrium?

(ix) A non-symmetric Nash equilibrium? (cf. [32, Section 2.3.1])

Some of these problems were first shown $\mathcal{N} \mathcal{P}$-hard for symmetric bimatrix games in the seminal paper by Gilboa and Zemel [20, Section 1.2]. Later Conitzer and Sandholm [13, Section 3] gave a unifying polynomial time reduction from CNF SAT to show in one shot $\mathcal{N} \mathcal{P}$-hardness results, encompassing those from [20], for symmetric bimatrix games; their reduction yields games with Nash equilibria mirroring satisfiability parsimoniously. McLennan and Tourky [25, Theorem 1] refined some of these $\mathcal{N} \mathcal{P}$-hardness results for imitation bimatrix games, where the utility of the imitator is 1 if and only if she chooses the same strategy as the mover [26]. The problems (vii) and (viii) are considered here for the first time; (viii) is trivial for symmetric games.

The present authors introduced the decision problem about the equivalence of the sets of Nash equilibria of two given games, which they proved co- $\mathcal{N} \mathcal{P}$-hard [4, Theorem 1]:

(x) Do the two given games have the same sets of Nash equilibria? 
An additional decision problem, which is trivial for bimatrix games, becomes $\mathcal{N} \mathcal{P}$-hard already for 3-player games [4, Theorem 2]:

(xi) A Nash equilibrium where all probabilities are rational? [4]

It is natural to ask whether these problems remain $\mathcal{N} \mathcal{P}$-hard when restricted to win-lose games. To the best of our knowledge, this important question has been addressed only in [7, 12]. It was shown in [12, Theorem 1] that the decision problem (i) with $k=1$ is $\mathcal{N} \mathcal{P}$-hard for win-lose bimatrix games; there so is a variant of (ii) for imitation win-lose bimatrix games. The decision problem (vi) was shown $\mathcal{N} \mathcal{P}$-hard for imitation win-lose bimatrix games in [7, Theorem 1].

The counting problem and the parity problem for Nash equilibria ask for the number and for the parity of the number of Nash equilibria, respectively, for a given game. To each decision problem there corresponds a counting problem and a parity problem, asking for the number and the parity of the number of Nash equilibria with the corresponding property, respectively. By the parsimonious property of the reduction in [13, these counting (resp., parity) problems are $\# \mathcal{P}$-hard (resp., $\oplus \mathcal{P}$-hard) for general bimatrix games; the $\# \mathcal{P}$-hardness (resp., $\oplus \mathcal{P}$-hardness) is inherited from the \#P्P-hardness [35] (resp., $\oplus \mathcal{P}$-hardness [33]) of computing the number (resp., the parity of the number) of satisfying assignments for a CNF SAT formula.

\subsubsection{State-of-the-Art and Statement of Results}

The polynomial time transformation of a general bimatrix game into a win-lose bimatrix game from [1] gave no guarantee on the preservation of properties of Nash equilibria; so, it had no implication on the complexity of deciding the properties for win-lose bimatrix games. Thus, the composition of a polynomial time reduction from an $\mathcal{N} \mathcal{P}$-hard problem to a decision problem about Nash equilibria for general bimatrix games (cf. [13, 20]) with the polynomial time transformation from [1] does not yield a polynomial time reduction from the $\mathcal{N} \mathcal{P}$-hard problem to the decision problems for win-lose bimatrix games, and their complexity remained open.

In this work, we settle the complexity of the decision problems about Nash equilibria 4, 7, 12, 13, 20, 25, 26, 28, for symmetric win-lose bimatrix games. Our main result is that these problems are $\mathcal{N} \mathcal{P}$-hard for symmetric win-lose bimatrix games (Theorems 7.1 and 7.7). Further, deciding the existence of a symmetric Nash equilibrium is $\mathcal{N} \mathcal{P}$-hard for win-lose bimatrix games (Theorem [7.9), and of a rational Nash equilibrium [4] is $\mathcal{N} \mathcal{P}$-hard for win-lose 3-player games (Theorem 7.10). As a byproduct, we derive, for symmetric win-lose bimatrix games, the $\mathcal{P} \mathcal{P} \mathcal{A D}$ hardness of the search problem (Theorem[6.7), the \#P-hardness of the counting problem and the $\oplus \mathcal{P}$-hardness of the parity problem (Theorem 6.8), and the $\# \mathcal{P}$-hardness and the $\oplus \mathcal{P}$-hardness of the counting and the parity version, respectively, of each decision problem (Theorem 7.1 ). 


\subsection{The Techniques}

We combine three powerful techniques: (1) Gadget games (Section 4). (2) A win-lose reduction (Section 5). (3) The classical GHR-symmetrization [21, with a new analysis tailored to winlose bimatrix games (Section 6). All three techniques involve the positive utility property: the property is enjoyed by the gadget games, which form a key component of the reduction, and it is required for the new analysis of the GHR-symmetrization. The property requires that each player may, in response to the choices of the other players, always choose a strategy to make her utility strictly positive; it is strictly weaker than the strictly positive utilities property, assumed for the analysis of the GHR-symmetrization in [22]. We prove that computing a Nash equilibrium for a win-lose bimatrix game with the property is as hard as computing a Nash equilibrium for a win-lose bimatrix game (Proposition 3.1); hence, it is $\mathcal{P} \mathcal{P} \mathcal{A D}$-hard. So assuming the positive utility property for symmetric win-lose bimatrix games does not simplify the search problem.

\subsubsection{Gadget Games}

A gadget game is a fixed game with a small number of players, which is void by design of the property associated with some decision problem about Nash equilibria (from Section 2.3). We present win-lose gadget games covering all such properties. For example, the win-lose 3-player irrational game $\widehat{G}_{2}$ (Section 4.2) has a single irrational Nash equilibrium, dismatching the problem $(x)$; the win-lose non-uniform game $\widehat{G}_{3}$ (Section 4.3) has no uniform Nash equilibrium, dismatching the problem (vi); the win-lose non-symmetric game $\widehat{G}_{4}$ (Section 4.4) has no symmetric Nash equilibrium, dismatching the problem (viii); for a given integer $k \geq 1$, the win-lose diagonal game $\widehat{G}_{5}[k]$ (Section 4.5) has exactly $k$ Nash equilibria, dismatching the problem (i).

\subsubsection{The Win-Lose Reduction}

The technical backbone of the complexity results for decision, counting and parity problems is a win-lose reduction we design, taking a fixed win-lose gadget game $\widehat{G}$ with the positive utility property as a parameter (Section 5). The reduction transforms a given 3SAT formula $\phi$ into a win-lose game $G=G(\widehat{G}, \phi) ; G$ and $\widehat{G}$ have the same number of players. We prove that each Nash equilibrium for $\widehat{G}$ is always a Nash equilibrium for $\mathrm{G}(\widehat{\mathrm{G}}, \phi)$ (Lemma 5.3 (Condition $(1))$ ), while $\mathrm{G}(\widehat{\mathrm{G}}, \phi)$ has additional Nash equilibria if and only if $\phi$ is satisfiable; those are related parsimoniously to the satisfying assignments of $\phi$. More important, each additional Nash equilibrium enjoys properties that do not depend on $\widehat{G}$ (Propositions 5.13 and 5.14). So a decision problem associated with some particular property reduces to deciding the inequivalence of a win-lose game with a fixed win-lose gadget game dismatching the property, and their equivalence is co- $\mathcal{N} \mathcal{P}$-hard. 


\subsubsection{The GHR-Symmetrization}

To extend hardness results from win-lose to symmetric win-lose bimatrix games, we seek winlose symmetrizations transforming a win-lose bimatrix game into a symmetric one. The GHRsymmetrization [21] is the single win-lose symmetrization we know of; for emphasis, we shall call it the win-lose GHR-symmetrization * We use tools from 22 to provide a new analysis of the GHR-symmetrization, tailored to win-lose bimatrix games with the positive utility property (Section 6), which yields a tight characterization of the Nash equilibria for the resulting symmetric win-lose bimatrix game $\widetilde{G}$ : they may only result, albeit in a non-parsimonious way, as balanced mixtures [22] involving Nash equilibria for G (Theorems 6.5 and 6.6) $\oplus$ By Propositions 5.13 and 5.14, the set of balanced mixtures is determined by the satisfiability of $\phi$; hence, the "cascade" of the win-lose reduction and the win-lose GHR symmetrization is a nonparsimonious reduction from 3SAT to decision problems about Nash equilibria in symmetric win-lose bimatrix games.

\subsection{Three-Steps Plan of the $\mathcal{N} \mathcal{P}$-Hardness Proof}

- Step 1: For a property of Nash equilibria for symmetric win-lose bimatrix games, fix a gadget game: a win-lose bimatrix game $\widehat{G}$ whose Nash equilibria dismatch the property.

- Step 2: Apply the win-lose reduction with parameter $\widehat{\mathrm{G}}$ on the formula $\phi$ to get the win-lose bimatrix game $\mathrm{G}:=\mathrm{G}(\widehat{\mathrm{G}}, \phi)$.

- When $\phi$ is unsatisfiable, the Nash equilibria for $\mathrm{G}(\widehat{\mathrm{G}}, \phi)$ are those for $\widehat{\mathrm{G}}$ (Proposition 5.131); hence, they dismatch the property.

- When $\phi$ is satisfiable, there are additional Nash equilbria for $\mathrm{G}(\widehat{\mathrm{G}}, \phi)$, which satisfy the property (Proposition 5.14). (It follows that the properties of the Nash equilibria for $\widehat{G}$ and of the additional ones for $\mathrm{G}(\widehat{\mathrm{G}}, \phi)$ are "conflicting".)

$\Rightarrow G$ has a Nash equilibrium matching the property if and only if $\phi$ is satisfiable. $\Rightarrow$ The associated decision problem is $\mathcal{N} \mathcal{P}$-hard for win-lose bimatrix games.

Note that Step 2 only allows proving the $\mathcal{N} \mathcal{P}$-hardness of decision problems associated with properties matched by the additional Nash equilibria for the win-lose game $\mathrm{G}(\widehat{\mathrm{G}}, \phi)$.

*The symmetrization due to Brown and von Neumann [8] involves the sum of the two matrices, which may increase the number of utility values. The symmetrization due to Gale, Kuhn and Tucker [17 introduces utilities -1 . So they both result in more than two values for the utilities, and none of the symmetrizations is win-lose.

${ }^{\dagger}$ The balanced mixture was introduced in [22] for the analysis of the GHR-symmetrization; it was later used in [23], where it was called the GKT-product, for the analysis of the GKT-symmetrization [17. 
- Step 3: Apply the win-lose GHR-symmetrization on G to get the symmetric win-lose bimatrix game $\widetilde{G}:=\operatorname{GHR}(\mathrm{G}(\widehat{\mathrm{G}}, \phi))$, whose Nash equilibria may only result as balanced mixtures involving Nash equilibria for $G$. Some balanced mixtures preserve the properties of the Nash equilibria for G, while other may dismatch them. This allows incorporating properties either matched or dismatched by the additional Nash equilibria for G. We show that the associated decision problem is $\mathcal{N} \mathcal{P}$-hard for symmetric win-lose bimatrix games by establishing a suitable equivalence to the satisfiability of $\phi$ as follows:

There are three possible cases in achieving the equivalence between the existence of a Nash equilibrium matching the property for $\widetilde{G}$ and the satisfiablity of $\phi$ :

(1) The existence of a Nash equilibrium matching the property for the win-lose game $\mathrm{G}$ is equivalent to the satisfiability of $\phi$, and two extra conditions hold:

* When $\phi$ is unsatisfiable, every balanced mixture dismatches the property.

* When $\phi$ is satisfiable, there is a balanced mixture matching the property.

In this case, the equivalence is preserved by the win-lose GHR-symmetrization.

(2) There is no Nash equilibrium for G matching the property, no matter whether $\phi$ is satisfiable or not, and two extra conditions hold:

* When $\phi$ is unsatisfiable, every balanced mixture dismatches the property.

* When $\phi$ is satisfiable, there is a balanced mixture matching the property.

Hence, the existence of a Nash equilibrium matching the property for $\widetilde{G}$ is equivalent to the satisfiability of $\phi$.

(3) When $\phi$ is unsatisfiable, there is a balanced mixture matching the property. When $\phi$ is satisfiable, there is an additional balanced mixture matching the property. Hence, this excludes the equivalence between the existence of a Nash equilibrium matching the property for $\widetilde{G}$ and the satisfiability of $\phi$.

We extend $\widetilde{\mathrm{G}}$ to $\widetilde{\mathrm{G}} \| \widehat{\mathrm{G}}_{5}[k]$ by "embedding" the symmetric win-lose gadget $\widehat{\mathrm{G}}_{5}[k]$ as a subgame so that the balanced mixtures arising when $\phi$ is unsatisfiable are "destroyed', while the balanced mixtures arising when $\phi$ is satisfiable are not "destroyed". This induces the equivalence of the existence of a Nash equilibrium matching the property for $\widetilde{G}_{\|} \widehat{G}_{5}[k]$ and the satisfiability of $\phi$.

$\Rightarrow$ Since equivalence to the satisfiability of $\phi$ holds in all cases, the $\mathcal{N} \mathcal{P}$-hardness of the associated decision problem for symmetric win-lose games follows. 


\subsection{The Complexity Results and Significance}

\subsubsection{Decision Problems}

The three-steps proof plan from Section 1.3 is used to yield, as our main result, the $\mathcal{N} \mathcal{P}$ hardness of deciding a handful of properties for symmetric win-lose bimatrix games (Theorems 7.1 and 7.7). These complexity results imply that symmetric win-lose bimatrix games are as complex as general bimatrix games with respect to the handful of decision problems about Nash equilibria considered before in [4, 17, 12, 13, 20, 25, 26, 28,.

While the win-lose reduction applies to games with any number $r \geq 2$ of players, the GHRsymmetrization is specific to bimatrix games. Hence, Step 1 and Step 2 suffice on their own for proving the $\mathcal{N} \mathcal{P}$-hardness of decision problems about Nash equilibria which either remain trivial for symmetric games (such as deciding the existence of a symmetric Nash equilibrium) or become non-trivial for win-lose games with more than two players (such as deciding the existence of a rational Nash equilibrium):

- Choosing $\widehat{G}$ as the win-lose bimatrix game $\widehat{G}_{5}$ with no symmetric Nash equilibrium yields the $\mathcal{N} \mathcal{P}$-hardness of deciding the existence of a symmetric Nash equilibrium for win-lose bimatrix games (Theorem (7.9). So win-lose bimatrix games are as complex as general bimatrix games with respect to deciding the existence of a symmetric Nash equilibrium.

- Choosing $\widehat{G}$ as the win-lose 3-player game $\widehat{G}_{2}$ with a single irrational Nash equilibrium yields the $\mathcal{N} \mathcal{P}$-hardness of deciding the existence of a rational Nash equilibrium for winlose 3-player games (Theorem 7.10). So win-lose 3-player games are as complex as general 3-player games with respect to deciding the existence of a rational Nash equilibrium.

These results represent an analog of the earlier result that win-lose bimatrix games are as complex as general bimatrix games with respect to the search problem for a Nash equilibrium [1].

\subsubsection{Search, Counting and Parity Problems}

We show that computing a Nash equilibrium for a symmetric win-lose bimatrix game is $\mathcal{P} \mathcal{P} \mathcal{A} \mathcal{D}$ hard (Theorem 6.7); the proof appeals to the characterization of the Nash equilibria for the win-lose GHR-symmetrization of a win-lose bimatrix game (Theorem 6.6).

Recall that the reduction used for the $\mathcal{N} \mathcal{P}$-hardness proof is non-parsimonious. Hence, the counting and parity problems about the number and the parity of the number of Nash equilibria for a symmetric win-lose bimatrix game, as well as the counting and parity versions of decision problems about Nash equilibria, are not immediately $\# \mathcal{P}$-hard and $\oplus \mathcal{P}$-hard, respectively. Nevertheless, the $\mathcal{N} \mathcal{P}$-hardness proof yields $\# \mathcal{P}$-hardness and $\oplus \mathcal{P}$-hardness results as well: 
- We show that computing the number (resp., the parity of the number) of Nash equilibria for a symmetric win-lose bimatrix game is \#P-hard (resp., $\oplus \mathcal{P}$-hard) (Theorem 6.8). The proof draws from simple formulas for the numbers of Nash equilibria for $G$ and $\widetilde{G}$ in terms of the numbers of Nash equilibria for $\widehat{G}$ and of satisfying assignments for $\phi$, denoted as $\# \phi$; the formulas follow from properties of the gadget games, the win-lose reduction and the win-lose GHR-symmetrization. Solving the formula for \# $\phi$ yields the $\# \mathcal{P}$-hardness; computing the parity of $\# \phi$, denoted as $\oplus \phi$, from the formula yields the $\oplus \mathcal{P}$-hardness.

- We examine the balanced mixtures of Nash equilibria for $G$ matching each property to derive simple formulas for the numbers of Nash equilibria for $\widetilde{G}$ matching the property. Hence, the counting versions of these decision problems are \#P-hard (Theorem 7.1).

Furthermore, all but two of the formulas are parity-preserving: the parity of the number of Nash equilibria for $\widetilde{G}$ matching the property yields $\oplus \phi$; this implies the $\oplus \mathcal{P}$-hardness of all but one of the parity versions (Theorem 7.1), the exception made by a trivial parity problem. In this sense, the "cascade" of the win-lose reduction and the win-lose GHR-symmetrization is parity-preserving: it yields the parity of the number of satisfying assignments. As far as we know, this is the first non-parsimonious, yet parity-preserving, reduction to decision problems about Nash equilibria.

\subsection{Evaluation and Comparison to Related Work}

None of the works [4, 7, 12, 13, 20, 25, 26, 28, on the complexity of decision and counting problems about Nash equilibria in bimatrix games considered the two restrictions to win-lose bimatrix and symmetric bimatrix games in simultaneity; neither did the works [1, 9, 10, 14] on the complexity of the search problem. This work encompasses all of the decision problems, together with their counting and parity versions, in the common framework composed of the gadget games, the win-lose reduction and the win-lose GHR-symmetrization. So, problemspecific reductions and techniques, such as the regular subgraphs technique from [7] or the good assignments technique from [12], are not necessary.

\subsubsection{Complexity Results}

Theorems 17.1, 7.7] and 7.10 improve and extend previous $\mathcal{N} \mathcal{P}$-hardness and co- $\mathcal{N} \mathcal{P}$-hardness results for the decision problems from [4, 7, 12, 13, 20, 25, 26, 28] as follows:

- The $\mathcal{N} \mathcal{P}$-hardness of the handful of decision problems improves the results in [13, 20, which applied to general symmetric bimatrix games, and extends their refinements in [25, [26], which applied to imitation bimatrix games. 
- The $\mathcal{N} \mathcal{P}$-hardness of deciding the existence of a uniform Nash equilibrium for a symmetric win-lose bimatrix game extends [7, Theorem 1], which applied to imitation win-lose bimatrix games.

- The $\mathcal{N} \mathcal{P}$-hardness of deciding the existence of $k+1$ Nash equilibria, with $k \geq 1$, for a symmetric win-lose bimatrix game improves [12, Theorem 1], which addressed the special case $k=1$ and applied to win-lose bimatrix games.

- The $\mathcal{N} \mathcal{P}$-hardness of deciding the existence of a non-symmetric Nash equilibrium for a symmetric win-lose bimatrix game improves [28, Theorem 3], which applied to general symmetric bimatrix games.

- The co- $\mathcal{N} \mathcal{P}$-hardness of deciding the Nash-equivalence of a given symmetric win-lose lose bimatrix game with the GHR-symmetrization of a fixed win-lose gadget game improves [4, Theorem 1], which established the Nash-equivalence of a given general bimatrix game with a fixed general gadget game.

- The $\mathcal{N} \mathcal{P}$-hardness of deciding the existence of a rational Nash equilibrium for a win-lose 3-player game improves [4, Theorem 2], which applied to general 3-player games.

Corresponding extensions and improvements follow for the $\# \mathcal{P}$-hardness of the counting versions of these decision problems. In particular:

- The \#P-hardness of counting the number of non-symmetric Nash equilibria for a symmetric win-lose bimatrix game improves [28, Theorem 5], which applied to general symmetric bimatrix games.

Of particular interest is the $\# \mathcal{P}$-hardness (resp., $\oplus \mathcal{P}$-hardness) of computing, for a given symmetric win-lose bimatrix game, the number (resp., the parity of the number) of symmetric Nash equilibria; recall that the corresponding decision problem, asking for the existence of a symmetric Nash equilibrium for a given symmetric game, is trivial by the early result of Nash [29, 30].

To the best of our knowledge, the proof in 36] that computing the parity of the number of satisfying assignments for a read-twice formula, where each variable occurs at most twice, is $\oplus \mathcal{P}$-hard is the only proof of $\oplus \mathcal{P}$-hardness employing a reduction from a corresponding $\mathcal{N} \mathcal{P}$-completeness proof which, although non-parsimonious, is parity-preserving.

Figure 4 tabulates the presented complexity results for decision problems in comparison to those in [4, 7, 12, 13, 20, 28]. Theorem 6.7 improves the $\mathcal{P} \mathcal{P} \mathcal{A} \mathcal{D}$-hardness of the search problem for win-lose bimatrix games from [1] to symmetric win-lose bimatrix games. The \#Phardness of computing the number of Nash equilibria for a symmetric win-lose bimatrix game in Theorem 6.8 improves [13, Corollary 12], which applied to general symmetric bimatrix games. 


\subsubsection{The Win-Lose Reduction}

The unifying reduction from CNF SAT, introduced in [13] and further developed in [4, 24, is inadequate to cover win-lose games. (See Section 5.1.1 for a technical discussion.) New ideas and technical constructs, such as pair variables, were needed for the win-lose reduction, which thus improves vastly in yielding a win-lose game while still preserving the equivalence between the existence of additional Nash equilibria for the game $\mathrm{G}(\widehat{\mathrm{G}}, \phi)$ and the satisfiability of $\phi$. We needed the finer structure of a 3SAT formula in order to guarantee that certain deviations in the game constructed by the win-lose reduction are non-profitable. Specifically, the proofs for Propositions 5.13 and 5.14 rely on choosing $\phi$ as a 3SAT formula in an essential way.

The win-lose reduction generalizes the reduction from [13], which yielded a bimatrix game, to yield an $r$-player game, with $r \geq 2$; it is this generalization that has enabled showing complexity results about decision problems, such as deciding the existence of a rational Nash equilibrium (Theorem 7.10), which are trivial for bimatrix games but become $\mathcal{N} \mathcal{P}$-hard for 3-player games.

We note that the reduction in [13] yielded the $\mathcal{N} \mathcal{P}$-hardness of approximate versions of some of the decision problems over general bimatrix games. We anticipate that the presented composition of the win-lose reduction and the win-lose GHR-symmetrization yields corresponding $\mathcal{N} \mathcal{P}$-hardness results over symmetric win-lose bimatrix games.

\subsubsection{The GHR-Symmetrization}

Our analysis of the GHR-symmetrization [21] yields the first complete characterization of the Nash equilibria for the symmetric game resulting from a win-lose symmetrization. For its previous analysis in 22], it was assumed that all utilities in the original bimatrix game were strictly positive in order to guarantee that the 0 utilities added for the GHR-symmetrization are strictly less than any utility; thus, the original bimatrix game were not a win-lose game. Recall that shifting and scaling the utilities does not alter the set of Nash equilibria for a game. Thus, a bimatrix game with at most two values for the (strictly positive) utilities could be transformed into an equivalent win-lose game by shifting and scaling the utilities. (Note that the case where all utilities in the original bimatrix games are equal is degenerate.) But then it is no more the case that the 0 utilities added for the GHR-symmetrization are strictly less than both 0 and 1, and the analysis of the GHR-symmetrization from [22] is no more applicable.

To circumvent this difficulty, we need a different assumption on the utilities in the original bimatrix game which is not too restrictive to make the algorithmic problems easier, while it is strong enough to yield a tight characterization of the Nash equilibria for the GHRsymmetrization; this is the positive utility property. We analyze the GHR-symmetrization for win-lose bimatrix games (Section 6), replacing the assumption of strictly positive utilities 
from [22] with the positive utility property. The resulting characterization of Nash equilibria for the GHR-symmetrization of a win-lose bimatrix game with the positive utility property is similar to the characterization of Nash equilibria for the GHR-symmetrization of a bimatrix game with strictly positive utilities in [22]: Cases $\left(C^{\prime} .2\right)$ and $\left(C^{\prime} .3\right)$ from Theorem 6.6 correspond to the cases addressed in [22, Theorem 4.1]; [22, Theorem 4.2] and [22, Theorem 4.3] correspond to Theorems 6.5 and 6.6, respectively.

\subsubsection{Tractable Cases}

Bilò and Fanelli [3, Section 4] consider a Linear Programming formulation, denoted as LR, of Nash equilibria in bimatrix games, which is a relaxation of a corresponding Quadratic Programming formulation they propose. They show [3, Theorem 1] that any feasible solution to $L R$ is a Nash equilibrium when the bimatrix game is regular [3, Definition 4]: the sum of corresponding entries in the two matrices remains constant either accross rows or across columns; so, regular bimatrix games are the rank-1 bimatrix games, encompassing zero-sum bimatrix games. As a consequence of [3, Theorem 1], a Nash equilibrium optimizing any objective function involving the players' utilities and meeting any set of constraints expressible through Linear Programming can be computed in polynomial time (through solving $L R$ ). Since nearly all decision problems about Nash equilibria studied in this work are so expressible, it follows that they are polynomial time solvable when restricted to regular bimatrix games. (A notable exception is problem (v) from Section 1.1.3, which remains $\mathcal{N} \mathcal{P}$-hard even when restricted to zero-sum games [20].) This positive result stands in contrast to the established $\mathcal{N} \mathcal{P}$-hardness for symmetric win-lose bimatrix games. Other positive results on the search problem for a Nash equilibrium in planar, sparse and $K_{3,3}$ and $K_{5}$ minor-free and minor-closed win-lose bimatrix games appear in [2, [1] and 15 , respectively.

\subsection{Paper Organization}

Section 2 introduces the game-theoretic framework. Section 3 considers the positive utility property for win-lose bimatrix games. The win-lose gadget games are presented in Section 4 . Section 5 treats the win-lose reduction and its properties. The win-lose GHR-symmetrization and its properties are analyzed in Section 6. Section 7 presents the complexity results. We conclude, in Section 8 , with a discussion of the results and some open problems. 


\section{Framework and Preliminaries}

Games, Nash equilibria and their decision problems are treated in Sections 2.1, 2.2 and 2.3, respectively.

\subsection{Games}

A game is a triple $\mathrm{G}=\left\langle[r],\left\{\Sigma_{i}\right\}_{i \in[r]},\left\{\mathrm{U}_{i}\right\}_{i \in[r]}\right\rangle$, where: (i) $[r]=\{1, \ldots, r\}$ is a finite set of players with $r \geq 2$, and (ii) for each player $i \in[r], \Sigma_{i}$ is the set of strategies for player $i$, and the utility function $\mathrm{U}_{i}$ is a function $\mathrm{U}_{i}: \times_{k \in[r]} \Sigma_{k} \rightarrow \mathbb{R}$ for player $i$. The game $\mathrm{G}$ is win-lose (resp., general) if for each player $i \in[r]$, the utility function is a function $\bigcup_{i}: \times_{k \in[r]} \Sigma_{k} \rightarrow\{0,1\}$ (resp., $\left.\mathrm{U}_{i}: \times_{k \in[r]} \Sigma_{k} \rightarrow \mathbb{Q}\right)$. For each player $i \in[r]$, denote $\Sigma_{-i}=\times_{k \in[r] \backslash\{i\}} \Sigma_{k}$; denote $\Sigma=\times_{k \in[r]} \Sigma_{k}$. For an integer $r \geq 2, r-\mathcal{G}$ is the set of $r$-player games; so, $\mathcal{G}=\bigcup_{r \geq 2} r-\mathcal{G}$ is the set of all games.

A profile is a tuple $\mathbf{s} \in \Sigma$ of $r$ strategies, one for each player. For a profile $\mathbf{s}$, the vector $\mathrm{U}(\mathbf{s})=\left\langle\mathrm{U}_{1}(\mathbf{s}), \ldots, \mathrm{U}_{r}(\mathbf{s})\right\rangle$ is called the utility vector. For a profile $\mathbf{s}$ and a strategy $t \in \Sigma_{i}$ of player $i$, denote as $\mathbf{s}_{-i} \diamond t$ the profile obtained by substituting $t$ for $s_{i}$ in $\mathbf{s}$. A partial profile $\mathbf{s}_{-i} \in \Sigma_{-i}$ is a tuple of $r-1$ strategies, one for each player other than $i$. We define:

Definition 2.1 The game $\mathrm{G}$ has the positive utility property if for each player $i \in[r]$ and each partial profile $\mathbf{s}_{-i} \in \Sigma_{-i}$, there is a strategy $t=t\left(\mathbf{s}_{-i}\right) \in \Sigma_{i}$ such that $\mathbf{U}_{i}\left(\mathbf{s}_{-i} \diamond t\right)>0$.

For win-lose bimatrix games, the positive utility property means that the utility matrix of the row (resp., column) player has no all-zeros column (resp., row).

A bimatrix game is a 2-player game with player 1, or row player, and player 2, or column player, with $\Sigma_{1}=\Sigma_{2}=[n]$ which is represented as the pair of matrices $\langle\mathrm{R}, \mathrm{C}\rangle$, where for each profile $\left\langle s_{1}, s_{2}\right\rangle \in \Sigma, \mathrm{U}_{1}\left(\left\langle s_{1}, s_{2}\right\rangle\right)=\mathrm{R}\left[s_{1}, s_{2}\right]$ and $\mathrm{U}_{2}\left(\left\langle s_{1}, s_{2}\right\rangle\right)=\mathrm{C}\left[s_{1}, s_{2}\right]$. The game $\mathrm{G}=\langle\mathrm{R}, \mathrm{C}\rangle$ is symmetric if $\mathrm{U}_{1}\left(\left\langle s_{1}, s_{2}\right\rangle\right)=\mathrm{U}_{2}\left(\left\langle s_{2}, s_{1}\right\rangle\right)$ : exchanging players and strategies preserves utilities; so, $\mathrm{R}=\mathrm{C}^{\mathrm{T}}$. For a constant $c$, the game $\mathrm{G}$ is $c$-sum if for each profile $\left\langle s_{1}, s_{2}\right\rangle, \mathrm{U}_{1}\left(\left\langle s_{1}, s_{2}\right\rangle\right)+$ $\mathrm{U}_{2}\left(\left\langle s_{1}, s_{2}\right\rangle\right)=c$; so, $\mathrm{R}\left[s_{1}, s_{2}\right]+\mathrm{C}\left[s_{1}, s_{2}\right]=c$. For a player $i \in[2]$ in the game $\mathrm{G}$, we shall denote as $\bar{i}$ the player other than $i$; so $\bar{i} \in[2] \backslash\{i\}$.

A mixed strategy for player $i \in[r]$ is a probability distribution $\sigma_{i}$ on her strategy set $\Sigma_{i}$ : a function $\sigma_{i}: \Sigma_{i} \rightarrow[0,1]$ such that $\sum_{s \in \Sigma_{i}} \sigma_{i}(s)=1$. Denote as $\operatorname{Supp}\left(\sigma_{i}\right)$ the set of strategies $s \in \Sigma_{i}$ such that $\sigma_{i}(s)>0$. The mixed strategy $\sigma_{i}: \Sigma_{i} \rightarrow[0,1]$ is pure, and player $i$ is pure,

\footnotetext{
${ }^{\ddagger}$ The assumption that the two players have equal numbers of strategies is without loss of generality since equality can be achieved, without altering the positive utility property, by adding "dummy" strategies, which are never played by a utility-maximizing player.
} 
if $\operatorname{Supp}\left(\sigma_{i}\right)=\{s\}$ for some strategy $s \in \Sigma_{i}$; player $i$ is mixed if she is not pure. The mixed strategy $\sigma_{i}: \Sigma_{i} \rightarrow[0,1]$ is fully mixed, and player $i$ is fully mixed, if $\operatorname{Supp}\left(\sigma_{i}\right)=\Sigma_{i}$; so, player $i$ puts non-zero probability on all strategies in her set of strategies $\Sigma_{i}$. The mixed strategy $\sigma_{i}: \Sigma_{i} \rightarrow[0,1]$ is uniform if for each pair of strategies $s, t \in \operatorname{Supp}\left(\sigma_{i}\right), \sigma_{i}(s)=\sigma_{i}(t)$. The mixed strategy $\sigma_{i}$ is rational if all values of $\sigma_{i}$ are rational numbers.

A mixed profile $\boldsymbol{\sigma}=\left(\sigma_{i}\right)_{i \in[r]}$ is a tuple of mixed strategies, one for each player. So, a profile is the degenerate case of a mixed profile where all probabilities are either 0 or 1 . A partial mixed profile $\boldsymbol{\sigma}_{-i}$ is a tuple of $r-1$ mixed strategies, one for each player other than $i$. For a mixed profile $\boldsymbol{\sigma}$ and a mixed strategy $\tau_{i}$ of player $i \in[r]$, denote as $\boldsymbol{\sigma}_{-i} \diamond \tau_{i}$ the mixed profile obtained by substituting $\tau_{i}$ for $\sigma_{i}$ in $\boldsymbol{\sigma}$. A mixed profile is uniform (resp., fully mixed) if all of its mixed strategies are uniform (resp., fully mixed); else it is non-uniform (resp., non-fully mixed). A mixed profile is symmetric if all mixed strategies are identical; else it is non-symmetric. A mixed profile is rational if all of its mixed strategies are rational; else it is irrational.

The mixed profile $\boldsymbol{\sigma}$ induces a probability measure $\mathbb{P}_{\boldsymbol{\sigma}}$ on the set of profiles in the natural way; so, for a profile $\mathbf{s}, \mathbb{P}_{\boldsymbol{\sigma}}(\mathbf{s})=\prod_{k \in[r]} \sigma_{k}\left(s_{k}\right)$. Say that the profile $\mathbf{s}$ is supported in the mixed profile $\boldsymbol{\sigma}$, and write $\mathbf{s} \sim \boldsymbol{\sigma}$, if $\mathbb{P}_{\boldsymbol{\sigma}}(\mathbf{s})>0$. Under the mixed profile $\boldsymbol{\sigma}$, the utility of each player becomes a random variable. So, associated with the mixed profile $\sigma$ is the expected utility $\mathrm{U}_{i}(\boldsymbol{\sigma})=\mathbb{E}_{\mathbf{s} \sim \boldsymbol{\sigma}}\left(\mathrm{U}_{i}(\mathbf{s})\right)$ for each player $i \in[r]$ : the expectation according to $\mathbb{P}_{\boldsymbol{\sigma}}$ of her utility; so,

$$
\mathrm{U}_{i}(\boldsymbol{\sigma})=\sum_{\mathbf{s} \in \Sigma}\left(\prod_{k \in[r]} \sigma_{k}\left(s_{k}\right)\right) \cdot \mathrm{U}_{i}(\mathbf{s}) .
$$

Recall that in a $c$-sum bimatrix game $\mathrm{G}$, for each mixed profile $\boldsymbol{\sigma}, \mathrm{U}_{1}(\boldsymbol{\sigma})+\mathrm{U}_{2}(\boldsymbol{\sigma})=c$. Also, in a symmetric bimatrix game, the mixed exchangeability property holds: exchanging players and mixed strategies preserves expected utilities in the sense that for any mixed profile $\left\langle\sigma_{1}, \sigma_{2}\right\rangle$, $\mathrm{U}_{1}\left(\left\langle s_{1}, s_{2}\right\rangle\right)=\mathrm{U}_{2}\left(\left\langle s_{2}, s_{1}\right\rangle\right) \S$

\subsection{Nash Equilibria}

A pure Nash equilibrium is a profile $\mathbf{s} \in \Sigma$ such that for each player $i \in[r]$ and for each strategy $t \in \Sigma_{i}, \mathbf{U}_{i}(\mathbf{s}) \geq \mathbf{U}_{i}\left(\mathbf{s}_{-i} \diamond t\right)$. A mixed Nash equilibrium, or Nash equilibrium for short, is a mixed profile $\boldsymbol{\sigma}$ such that for each player $i \in[r]$ and for each mixed strategy $\tau_{i}, \mathbf{U}_{i}(\boldsymbol{\sigma}) \geq \mathbf{U}_{i}\left(\boldsymbol{\sigma}_{-i} \diamond \tau_{i}\right)$. Note that the mixed exchangeability property of symmetric bimatrix games implies that (mixed) Nash equilibria are preseved in a symmetric bimatrix game: $\left\langle\sigma_{1}, \sigma_{2}\right\rangle$ is a Nash equilibrium if and only if $\left\langle\sigma_{2}, \sigma_{1}\right\rangle$ is. Denote as $\mathcal{N} \mathcal{E}(\mathrm{G})$ (resp., $\mathcal{S} \mathcal{N} \mathcal{E}(\mathrm{G})$ ) the set of Nash equilibria (resp., symmetric Nash equilibria) for the game $G$. For each game $G, \mathcal{N E}(G) \neq \emptyset[29$, 30]; for each

\footnotetext{
${ }^{\S}$ Indeed, $\quad \mathrm{U}_{1}\left(\left\langle\sigma_{1}, \sigma_{2}\right\rangle\right)=\sum_{s_{1}, s_{2} \in[n]} \sigma_{1}\left(s_{1}\right) \sigma_{2}\left(s_{2}\right) \cdot \mathrm{R}\left[s_{1}, s_{2}\right]=\sum_{s_{1}, s_{2} \in[n]} \sigma_{1}\left(s_{1}\right) \sigma_{2}\left(s_{2}\right) \cdot \mathrm{C}^{\mathrm{T}}\left[s_{1}, s_{2}\right]=$ $\sum_{s_{1}, s_{2} \in[n]} \sigma_{1}\left(s_{1}\right) \sigma_{2}\left(s_{2}\right) \cdot \mathrm{C}\left[s_{2}, s_{1}\right]=\mathrm{U}_{2}\left(\left\langle\sigma_{2}, \sigma_{1}\right\rangle\right)$.
} 
symmetric game $\mathrm{G}, \mathcal{S} \mathcal{N} \mathcal{E}(\mathrm{G}) \neq \emptyset[29$, 30]. Two $r$-player games $\widehat{\mathrm{G}}$ and $\mathrm{G}$ are Nash-equivalent [4] if $\mathcal{N} \mathcal{E}(\widehat{\mathrm{G}})=\mathcal{N} \mathcal{E}(\mathrm{G})$; that is, they have the same set of Nash equilibria. We shall make extensive use of the following basic property of Nash equilibria.

Lemma 2.1 A mixed profile $\boldsymbol{\sigma}$ is a Nash equilibrium if and only if for each player $i \in[r],(1)$ for each strategy $t \in \operatorname{Supp}\left(\sigma_{i}\right), \mathbf{U}_{i}(\boldsymbol{\sigma})=\mathbf{U}_{i}\left(\boldsymbol{\sigma}_{-i} \diamond t\right)$, and (2) for each strategy $t \notin \operatorname{Supp}\left(\sigma_{i}\right)$, $\mathrm{U}_{i}(\boldsymbol{\sigma}) \geq \mathrm{U}_{i}\left(\boldsymbol{\sigma}_{-i} \diamond t\right)$.

Given a partial mixed profile $\boldsymbol{\sigma}_{-i}$ for some player $i \in[r]$, a best-response for player $i$ to $\boldsymbol{\sigma}_{-i}$ is a pure strategy $s \in \Sigma_{i}$ such that $\mathrm{U}_{i}\left(\boldsymbol{\sigma}_{-i} \diamond s\right)=\max _{t \in \Sigma_{i}} \mathrm{U}_{i}\left(\boldsymbol{\sigma}_{-i} \diamond t\right)$. Lemma 2.1 (Condition (2)) immediately implies that in a Nash equilibrium $\boldsymbol{\sigma}$, for each player $i \in[r]$ and strategy $s \in \Sigma_{i}$, $s \in \operatorname{Supp}\left(\sigma_{i}\right)$ only if $s$ is a best-response for player $i$ to $\boldsymbol{\sigma}_{-i}$. Lemma 2.1 and the positive utility property immediately imply:

Lemma 2.2 Fix a win-lose game G with the positive utility property. Then, in a Nash equilibrium $\boldsymbol{\sigma}$, for each player $i \in[r], \mathrm{U}_{i}(\boldsymbol{\sigma})>0$.

Proof: Assume, by way of contradiction, that $\mathbf{U}_{i}(\boldsymbol{\sigma})=0$. Choose a partial profile $\mathbf{s}_{-i}$ supported in $\boldsymbol{\sigma}_{-i}$; so, $\mathbb{P}_{\boldsymbol{\sigma}_{-i}}\left(\mathbf{s}_{-i}\right)>0$. By the positive utility property, there is a strategy $t\left(\mathbf{s}_{-i}\right) \in \Sigma_{i}$ with $\mathrm{U}_{i}\left(\mathbf{s}_{-i} \diamond t\left(\mathbf{s}_{-i}\right)\right)>0$. Since $\mathrm{G}$ is win-lose,

$$
\mathrm{U}_{i}\left(\boldsymbol{\sigma}_{-i} \diamond t\left(\mathbf{s}_{-i}\right)\right) \geq \mathbb{P}_{\boldsymbol{\sigma}_{-i}}\left(\mathbf{s}_{-i}\right) \cdot \bigcup_{i}\left(\boldsymbol{\sigma}_{-i} \diamond t\left(\mathbf{s}_{-i}\right)\right)>0=\mathrm{U}_{i}(\boldsymbol{\sigma}) .
$$

A contradiction to Lemma 2.1.

We conclude with a simple property of Nash equilibria for win-lose games.

Lemma 2.3 Fix a win-lose bimatrix game $\langle\mathrm{R}, \mathrm{C}\rangle$ with a Nash equilibrium $\boldsymbol{\sigma} \in \mathcal{N} \mathcal{E}(\langle\mathrm{R}, \mathrm{C}\rangle)$ such that $\mathrm{U}_{i}(\boldsymbol{\sigma})=0$ for some player $i \in[2]$. Then, $\langle\mathrm{R}, \mathrm{C}\rangle$ has a pure Nash equilibrium.

Proof: For simpler notation, assume, without loss of generality, that $i=1$. Denote

$$
\bar{s}:=\operatorname{argmax}_{s_{2} \in \operatorname{Supp}\left(\sigma_{2}\right)} \max _{s_{1} \in \operatorname{Supp}\left(\sigma_{1}\right)}\left\{\mathrm{U}_{2}\left(\left\langle s_{1}, s_{2}\right\rangle\right)\right\} ;
$$

so, $\bar{s}$ is the strategy in $\operatorname{Supp}\left(\sigma_{2}\right)$ that incurs the maximum possible utility to player 2 over all strategies $s_{1}$ from $\operatorname{Supp}\left(\sigma_{1}\right)$ chosen by player 1 . Choose an arbitrary strategy $s^{*} \in \operatorname{Supp}\left(\sigma_{1}\right)$. We shall prove that the profile $\left\langle s^{*}, \bar{s}\right\rangle$ is a pure Nash equilibrium for $\langle\mathrm{R}, \mathrm{C}\rangle$. Since $\boldsymbol{\sigma}$ is a Nash equilibrium for $\langle\mathrm{R}, \mathrm{C}\rangle$, Lemma 2.1 (Condition (2)) implies that for each strategy $s \in \Sigma_{1}$, $\mathrm{U}_{1}\left(\boldsymbol{\sigma}_{-1} \diamond s\right) \leq \mathrm{U}_{1}(\boldsymbol{\sigma})=0$. Since $\langle\mathrm{R}, \mathrm{C}\rangle$ is win-lose, it follows that $\mathrm{U}_{1}\left(\boldsymbol{\sigma}_{-1} \diamond s\right)=0$, which implies that for each strategy $s \in \Sigma_{1}, \mathrm{U}_{1}\left(\left\langle s, s_{2}\right\rangle\right)=0$ for all strategies $s_{2} \in \operatorname{Supp}\left(\sigma_{2}\right)$. Since $\bar{s} \in$ $\operatorname{Supp}\left(\sigma_{2}\right)$, it follows that for each strategy $s \in \Sigma_{1}, \mathbf{U}_{1}(\langle s, \bar{s}\rangle)=0$. In particular, $\mathrm{U}_{1}\left(\left\langle s^{*}, \bar{s}\right\rangle\right)=0$, so that player 1 cannot improve by deviating from $s^{*}$. By the definition of $\bar{s}$, player 2 cannot improve either by deviating from $\bar{s}$. Hence, $\left\langle s^{*}, \bar{s}\right\rangle$ is a pure Nash equilibrium for $\langle\mathrm{R}, \mathrm{C}\rangle$. 


\subsection{Decision Problems about Nash Equilibria}

We shall assume some basic familiarity of the reader with the basic notions of $\mathcal{N} \mathcal{P}$-completeness, as outlined, for example, in [18]. All decision problems will be stated in the style of [18], where I. and Q. stand for Instance and Question, respectively. They are categorized in six groups.

Group I- Question about cardinality:

$\exists k+1 \mathrm{NASH}$ (with $k \geq 1$ )

I.: $\quad$ A game $\mathrm{G}$.

Q.: Does $\mathrm{G}$ have at least $k+1$ Nash equilibria?

Group II - Questions about the expected utilities:

$\exists$ NASH WITH LARGE UTILITIES

I.: $\quad$ A game $G$ and a number $u$.

Q.: Is there a Nash equilibrium $\boldsymbol{\sigma}$ such that for each player $i \in[r], \mathrm{U}_{i}(\boldsymbol{\sigma}) \geq u$ ?

$\exists$ NASH WITH SMALL UTILITIES

I.: $\quad$ A game $\mathrm{G}$ and a number $u$.

Q.: Is there a Nash equilibrium $\boldsymbol{\sigma}$ such that for each player $i \in[r], \mathrm{U}_{i}(\boldsymbol{\sigma}) \leq u$ ?

$\exists$ NASH WITH LARGE TOTAL UTILITY

I.: $\quad$ A game $\mathrm{G}$ and a number $u$.

Q.: $\quad$ Is there a Nash equilibrium $\boldsymbol{\sigma}$ such that $\sum_{i \in[r]} \mathrm{U}_{i}(\boldsymbol{\sigma}) \geq u$ ?

$\exists$ NASH WITH SMALL TOTAL UTILITY

I.: $\quad$ A game $G$ and a number $u$.

Q.: Is there a Nash equilibrium $\boldsymbol{\sigma}$ such that $\sum_{i \in[r]} \bigcup_{i}(\boldsymbol{\sigma}) \leq u$ ?

Group III-Questions about the supports:

$\exists$ NASH WITH LARGE SUPPORTS

I.: $\quad$ A game $\mathrm{G}$ and an integer $k \geq 1$.

Q.: Is there a Nash equilibrium $\boldsymbol{\sigma}$ such that for each player $i \in[r],\left|\operatorname{Supp}\left(\sigma_{i}\right)\right| \geq k$ ?

$\exists$ NASH WITH SMALL SUPPORTS

I.: $\quad$ A game $\mathrm{G}$ and an integer $k \geq 1$.

Q.: Is there a Nash equilibrium $\boldsymbol{\sigma}$ such that for each player $i \in[r],\left|\operatorname{Supp}\left(\sigma_{i}\right)\right| \leq k$ ?

$\exists$ NASH WITH RESTRICTING SUPPORTS 
I.: $\quad$ A game $\mathrm{G}$ and a subset of strategies $\mathrm{T}_{i} \subseteq \Sigma_{i}$ for each player $i \in[r]$.

Q.: Is there a Nash equilibrium $\boldsymbol{\sigma}$ such that for each player $i \in[r], \mathrm{T}_{i} \subseteq \operatorname{Supp}\left(\sigma_{i}\right)$ ?

$\exists$ NASH WITH RESTRICTED SUPPORTS

I.: $\quad$ A game $\mathrm{G}$ and a subset of strategies $\mathrm{T}_{i} \subseteq \Sigma_{i}$ for each player $i \in[r]$.

Q.: Is there a Nash equilibrium $\boldsymbol{\sigma}$ such that for each player $i \in[r], \operatorname{Supp}\left(\sigma_{i}\right) \subseteq \mathrm{T}_{i}$ ?

Group IV-Questions about refinements of Nash equilibrium:

A Nash equilibrium $\boldsymbol{\sigma}$ is Pareto-Optimal if for each mixed profile $\widehat{\boldsymbol{\sigma}}$ where there is a player $i \in[r]$ with $\mathbf{U}_{i}(\widehat{\boldsymbol{\sigma}})>\mathbf{U}_{i}(\boldsymbol{\sigma})$, there is a player $j \in[r]$ such that $\mathbf{U}_{j}(\widehat{\boldsymbol{\sigma}})<\mathbf{U}_{j}(\boldsymbol{\sigma})$; so, loosely speaking, there is no other mixed profile where at least one player is strictly better off and every player is at least as well off. Denote as Diff $(\boldsymbol{\sigma}, \widehat{\boldsymbol{\sigma}}):=\left\{i \in[r]: \sigma_{i} \neq \widehat{\sigma}_{i}\right\}$ the set of players with different mixed strategies in the mixed profiles $\boldsymbol{\sigma}$ and $\widehat{\boldsymbol{\sigma}}$. A Nash equilibrium $\boldsymbol{\sigma}$ is Strongly Pareto-Optimal if for each mixed profile $\widehat{\boldsymbol{\sigma}}$ with a player $i \in[r]$ such that $\mathrm{U}_{i}(\widehat{\boldsymbol{\sigma}})>\mathrm{U}_{i}(\boldsymbol{\sigma})$, there is a player $j \in \operatorname{Diff}(\boldsymbol{\sigma}, \widehat{\boldsymbol{\sigma}})$ such that $\mathbf{U}_{j}(\widehat{\boldsymbol{\sigma}}) \leq \mathbf{U}_{j}(\boldsymbol{\sigma})$; so, loosely speaking, there is no other mixed profile where at least one player is strictly better off and every player with a different mixed strategy is strictly better off.

\section{$\exists$ PARETO-OPTIMAL NASH}

I.: $\quad$ A game $\mathrm{G}$.

Q.: Is there a Pareto-Optimal Nash equilibrium?

$\exists \neg$ PARETO-OPTIMAL NASH

I.: A game $\mathrm{G}$.

Q.: Is there a Nash equilibrium which is not Pareto-Optimal?

$\exists$ STRONGLY PARETO-OPTIMAL NASH

I.: A game $\mathrm{G}$.

Q.: Is there a Strongly Pareto-Optimal Nash equilibrium?

$\exists \neg$ STRONGLY PARETO-OPTIMAL NASH

I.: $\quad$ A game $\mathrm{G}$.

Q.: Is there a Nash equilibrium which is not Strongly Pareto-Optimal?

Group $V$ - Questions about the probabilities:

\section{$\exists$ NASH WITH SMALL PROBABILITIES}

I.: A game $\mathrm{G}$.

Q.: $\quad$ Is there a Nash equilibrium $\boldsymbol{\sigma}$ such that for each player $i \in[r], \max _{s \in \Sigma_{i}} \sigma_{i}(s) \leq \frac{1}{2}$ ?

\section{$\exists$ UNIFORM NASH}


I.: $\quad$ A game $\mathrm{G}$.

Q.: Does $G$ have a uniform Nash equilibrium?

$\exists \neg$ UNIFORM NASH

I.: $\quad$ A game $G$.

Q.: Does $G$ have a non-uniform Nash equilibrium?

$\exists$ SYMMETRIC NASH

I.: $\quad$ A game $\mathrm{G}$.

Q.: Does $G$ have a symmetric Nash equilibrium?

$\exists \neg$ SYMMETRIC NASH

I.: $\quad$ A game $\mathrm{G}$.

Q.: Does G have a non-symmetric Nash equilibrium?

$\exists$ RATIONAL NASH

I.: $\quad$ A game $\mathrm{G}$.

Q.: Does G have a rational Nash equilibrium?

Restricted to general bimatrix games, all previous decision problems belong to $\mathcal{N} \mathcal{P}$ : given the polynomial-length supports for a mixed profile, it is polynomial time verifiable that it is a Nash equilibrium satisfying the property associated with the decision problem. $\exists$ RATIONAL NASH is trivial for general bimatrix games, and $\exists$ SYMMETRIC NASH is trivial for symmetric games. Note that $\exists$ UNIFORM NASH and $\exists$ RATIONAL NASH belong to $\mathcal{N} \mathcal{P}$ for any number of players.

Each of the previous decision problems has a corresponding cardinality or counting version: the problem of computing the number of Nash equilibria witnessing the validity of QUESTION Note that for general bimatrix games, the counting versions of all previous decision problems belong to \#P (thanks again to the efficient verifiability property of Nash equilibria). Furthermore, each of the decision problems has a parity version: the problem of computing the parity of the number of Nash equilibria witnessing the validity of QUESTION. The parity versions of all previous decision problems belong to $\oplus \mathcal{P} \|$ We shall use \# (resp., $\oplus$ ) in the place of $\exists$ to denote the counting (resp., parity) versions; for example, \# RATIONAL NASH (resp., $\oplus$ RATIONAL NASH) denotes the counting (resp., parity) version of $\exists$ RATIONAL NASH. Clearly, by the mixed exchangeability property of symmetric bimatrix games, the number of

\footnotetext{
ฯ $\exists k+1 \mathrm{NASH}$ has no counting version as it is already defined with a cardinality question.

$\| \oplus \mathcal{P}$, read as Parity $\oplus \mathcal{P}$, is the complexity class formalizing the question of the parity of the number of solutions to a combinatorial problem. Formally, $\oplus \mathcal{P}$ is the class of sets $S$ such that there is a nondeterministic Turing machine which, on input $x$, has an odd number of accepting computations if and only if $x \in S$. We shall adopt a definition of $\oplus \mathcal{P}$-completeness using polynomial-time many-to-one reductions. The development of the theory of $\oplus \mathcal{P}$-complete parity problems is rather limited — see the discussion in [36].
} 
non-symmetric Nash equilibria in a symmetric bimatrix game is even; this is because $\left\langle\sigma_{1}, \sigma_{2}\right\rangle$ is a non-symmetric Nash equilibrium if and only if $\left\langle\sigma_{2}, \sigma_{1}\right\rangle$ is. Hence, $\oplus \neg$ SYMMETRIC NASH for symmetric bimatrix games is in $\mathcal{P}$.

Group VI-Equivalence property:

\section{NASH-EQUIVALENCE}

I.: $\quad$ Two games $\widehat{G}$ and $\mathrm{G}$ from $r$-G, for some integer $r \geq 2$.

Q.: Are $\widehat{G}$ and $G$ Nash-equivalent?

For a fixed game $\widehat{G}$, called the gadget game, a parameterized restriction of NASH-EQUIVALENCE with a single input (the game $G$ ) results.

NASH-EQUIVALENCE $(\widehat{G})$

I.: $\quad$ A game $\mathrm{G}$ from $r-\mathcal{G}$ (where $\widehat{\mathrm{G}}$ is from $r-\mathcal{G})$.

Q.: Are $\widehat{G}$ and $G$ Nash-equivalent?

Restricted to general bimatrix games, NASH-EQUIVALENCE and NASH-EQUIVALENCE $(\widehat{G})$ belong to co- $\mathcal{N} \mathcal{P}$ : given the polynomial-length supports for a mixed profile, it is polynomial time verifiable that it is a Nash equilibrium for exactly one of the two games.

Matching $\mathcal{N} \mathcal{P}$ - and co- $\mathcal{N} \mathcal{P}$-hardness results for the decision problems above are later summarized in Figure 4 .

\section{Win-Lose Bimatrix Games with the Positive Utility Property}

We now prove that assuming the positive utility property does not simplify the search problem for a win-lose bimatrix game. Fix a win-lose bimatrix game $\langle\mathrm{R}, \mathrm{C}\rangle$. We start with a preliminary definition. For a player $i \in[2]$, a strategy $\bar{s} \in \Sigma_{\bar{i}}$ is an all-zeros counter-strategy against player $i$ if for every profile $\left\langle s_{1}, s_{2}\right\rangle \in \Sigma$ with $s_{\bar{i}}=\bar{s}, \mathrm{U}_{i}\left(\left\langle s_{1}, s_{2}\right\rangle\right)=0$; that is, an all-zeros counter-strategy against the row (resp., column) player is a column (resp., row) of $\mathrm{R}$ (resp., C) made up only of zero entries. Clearly, the positive utility property excludes all-zeros counter-strategies. We prove:

Proposition 3.1 Fix a win-lose bimatrix game $\langle\mathrm{R}, \mathrm{C}\rangle$. Then, one of two conditions holds:

(C.1) $\langle\mathrm{R}, \mathrm{C}\rangle$ has a pure Nash equilibrium.

(C.2) There is a polynomial time constructible win-lose bimatrix game $\langle\overline{\mathrm{R}}, \overline{\mathrm{C}}\rangle$ with the positive utility property such that $\mathcal{N} \mathcal{E}(\langle\overline{\mathrm{R}}, \overline{\mathrm{C}}\rangle)=\mathcal{N} \mathcal{E}(\langle\mathrm{R}, \mathrm{C}\rangle)$. 
Proof: If Condition (C.1) holds, then we are done. So assume that Condition (C.1) does not hold. Note that if at least one of $R$ and $C$ is the null matrix, then $\langle R, C\rangle$ has a pure Nash equilibrium and Condition (C.1) holds. It follows that none of $\mathrm{R}$ and $\mathrm{C}$ is the null matrix. Denote as $c \geq 0$ and $r \geq 0$ the number of all-zeros counter-strategies against the row and the column player, respectively, in the game $\langle\mathrm{R}, \mathrm{C}\rangle$. Note that $\langle\mathrm{R}, \mathrm{C}\rangle$ has the positive utility property if and only if $r=c=0$. Renumber now the players' strategies so that the first $r$ strategies for player 1 are the all-zeros counter-strategies against the column player, and the first $c$ strategies for player 2 are the all-zeros counter-strategies against the row player. Construct from $\langle R, C\rangle$ a win-lose bimatrix game $\langle\bar{R}, \bar{C}\rangle$ as follows:

(0) If $r=c=0$, then $\langle\overline{\mathrm{R}}, \overline{\mathrm{C}}\rangle:=\langle\mathrm{R}, \mathrm{C}\rangle$.

(1) Otherwise, add a new strategy $n+1$ to the strategy set of each player and set:

(1.0) $\overline{\mathrm{U}}(\langle n+1, n+1\rangle)=\langle 0,0\rangle$.

(1.1) $\overline{\mathrm{U}}\left(\left\langle n+1, s_{2}\right\rangle\right)=\langle 1,0\rangle$ for $s_{2} \leq c$, or $\langle 0,1\rangle$ for $c+1 \leq s_{2} \leq n$.

(1.2) $\overline{\mathrm{U}}\left(\left\langle s_{1}, n+1\right\rangle\right)=\langle 0,1\rangle$ for $s_{1} \leq r$, or $\langle 1,0\rangle$ for $r+1 \leq s_{1} \leq n$.

Clearly, this is a polynomial time construction. We prove:

Lemma 3.2 The game $\langle\overline{\mathrm{R}}, \overline{\mathrm{C}}\rangle$ has the positive utility property.

Proof: Note that in Case (0), the game $\langle\bar{R}, \bar{C}\rangle$ has the positive utility property by definition. So assume we are in Case (1). Consider the row matrix $\bar{R}$; we need to prove that it does not contain an all-zeros column. By Step (1.2) in the construction, the column $n+1$ of $\overline{\mathrm{R}}$ has an entry equal to 1 if and only if $r+1 \leq n$; the latter holds since $\mathrm{R}$ is not the null matrix. So it remains to consider the columns of $\overline{\mathrm{R}}$ that are inherited from $\mathrm{R}$. By the definition of $c$, every column $j$ of $\mathrm{R}$ with $c+1 \leq j \leq n$ is not all-zeros; for a column $j \in[c]$ of $\mathrm{R}, \overline{\mathbf{U}}_{1}(n+1, j)=1$ by Step (1.1) in the construction, and we are done. We use corresponding arguments to prove that the column matrix $\overline{\mathrm{C}}$ does not contain an all-zeros row.

Furthermore, Condition (C.2) holds vacuously in Case (0). Note also that in Case (1), $\langle\bar{R}, \bar{C}\rangle$ has no pure Nash equilibrium in which some player chooses strategy $n+1$. We proceed by case analysis. Assume first that there is a Nash equilibrium $\overline{\boldsymbol{\sigma}}$ for $\langle\overline{\mathrm{R}}, \overline{\mathrm{C}}\rangle$ such that at least one player $\bar{i} \in[2]$ plays with positive probability an all-zeros counter-strategy $\bar{s}$ against player $i$. By Lemma 2.1, choosing the strategy $\bar{s}$ with probability 1 is a best-response of player $\bar{i}$ to $\overline{\boldsymbol{\sigma}}_{-\bar{i}}$; so, player $\bar{i}$ cannot improve her utility when choosing the strategy $\bar{s}$ with probability 1 . Now, by the definition of an all-zeros counter-strategy, player $i$ gets utility 0 in the mixed profile 
$\overline{\boldsymbol{\sigma}}_{-\bar{i}} \diamond \bar{s}$, which she could not improve by switching to another mixed strategy. Hence, $\overline{\boldsymbol{\sigma}}_{-\bar{i}} \diamond \bar{s}$ is a Nash equilibrium with $\overline{\mathrm{U}}_{i}\left(\overline{\boldsymbol{\sigma}}_{-\bar{i}} \diamond \bar{s}\right)=0$. By Lemma 2.3, it follows that $\langle\overline{\mathrm{R}}, \overline{\mathrm{C}}\rangle$ has a pure Nash equilibrium s. Since $\langle\bar{R}, \bar{C}\rangle$ has no pure Nash equilibrium in which some player plays strategy $n+1, \mathrm{~s}$ is also a pure Nash equilibrium for $\langle\mathrm{R}, \mathrm{C}\rangle$. A contradiction.

Assume now that in each Nash equilibrium $\overline{\boldsymbol{\sigma}}$ for $\langle\overline{\mathrm{R}}, \overline{\mathrm{C}}\rangle$, no player $\bar{i}$ plays an all-zeros counterstrategy against player $i$; that is, $\operatorname{Supp}\left(\bar{\sigma}_{1}\right) \subseteq\{r+1, \ldots, n+1\}$ and $\operatorname{Supp}\left(\bar{\sigma}_{2}\right) \subseteq\{c+1, \ldots, n+1\}$. We first prove that no Nash equilibrium for $\langle\mathrm{R}, \mathrm{C}\rangle$ is "destroyed" due to adding strategy $n+1$.

Lemma 3.3 It holds that $\mathcal{N} \mathcal{E}(\langle\mathrm{R}, \mathrm{C}\rangle) \subseteq \mathcal{N} \mathcal{E}(\langle\overline{\mathrm{R}}, \overline{\mathrm{C}}\rangle)$.

Proof: Assume, by way of contradiction, that there is a Nash equilibrium $\boldsymbol{\sigma} \in \mathcal{N} \mathcal{E}(\langle\mathrm{R}, \mathrm{C}\rangle)$ such that $\sigma \notin \mathcal{N E}(\langle\bar{R}, \bar{C}\rangle$. By the construction of the game $\langle\bar{R}, \bar{C}\rangle$, this implies that there is player $i \in[2]$ with $\overline{\mathbf{U}}_{i}\left(\boldsymbol{\sigma}_{-i} \diamond(n+1)\right)>\mathbf{U}_{i}(\boldsymbol{\sigma})$. Since the game $\langle\overline{\mathrm{R}}, \overline{\mathrm{C}}\rangle$ is win-lose, this implies that $\overline{\mathbf{U}}_{i}\left(\boldsymbol{\sigma}_{-i} \diamond(n+1)\right)>0$. Since $\operatorname{Supp}\left(\sigma_{1}\right) \subseteq\{r+1, \ldots, n\}$ and $\operatorname{Supp}\left(\sigma_{2}\right) \subseteq\{c+1, \ldots, n\}$, it follows that any profile supported in $\sigma_{-i} \diamond(n+1)$ falls into either Case (1.1) with $i=1$ and $c+1 \leq s_{2} \leq n$ or into Case (1.2) with $i=2$ and $r+1 \leq s_{1} \leq n$. By construction, this implies that $\overline{\mathrm{U}}_{i}\left(\boldsymbol{\sigma}_{-i} \diamond(n+1)\right)=0$ for each player $i \in[2]$. A contradiction.

We now prove that no new Nash equilibrium for $\langle\bar{R}, \bar{C}\rangle$ is "created" due to adding strategy $n+1$.

Lemma 3.4 It holds that $\mathcal{N} \mathcal{E}(\langle\overline{\mathrm{R}}, \overline{\mathrm{C}}\rangle) \backslash \mathcal{N} \mathcal{E}(\langle\mathrm{R}, \mathrm{C}\rangle)=\emptyset$.

Proof: Assume, by way of contradiction, that there is a Nash equilibrium $\overline{\boldsymbol{\sigma}} \in \mathcal{N} \mathcal{E}(\langle\overline{\mathrm{R}}, \overline{\mathrm{C}}\rangle) \backslash$ $\mathcal{N} \mathcal{E}(\langle\mathrm{R}, \mathrm{C}\rangle)$. Since $\overline{\boldsymbol{\sigma}} \notin \mathcal{N} \mathcal{E}(\langle\mathrm{R}, \mathrm{C}\rangle)$, it must be that $\bar{\sigma}_{1}(n+1)+\bar{\sigma}_{2}(n+1)>0$. Assume that $\bar{\sigma}_{1}(n+1)>0$ and consider player 1 switching to strategy $n+1$. Since $\operatorname{Supp}\left(\bar{\sigma}_{2}\right) \subseteq$ $\{c+1, \ldots, n+1\}$, it follows that only profiles falling into Case (0) or Case (1.1) with $c+1 \leq s_{2} \leq n$ are supported by $\overline{\boldsymbol{\sigma}}_{-1} \diamond(n+1)$, which implies, by the construction, that $\overline{\mathbf{U}}_{1}\left(\overline{\boldsymbol{\sigma}}_{-1} \diamond(n+1)\right)=0$. Since $\overline{\boldsymbol{\sigma}}$ is a Nash equilibrium, Lemma 2.1 (Condition (1)) implies that $\overline{\mathrm{U}}_{1}(\overline{\boldsymbol{\sigma}})=0$. Hence, Lemma 2.3 implies that $\langle\bar{R}, \bar{C}\rangle$ has a pure Nash equilibrium $\left\langle s_{1}, s_{2}\right\rangle$. Since $\langle\bar{R}, \bar{C}\rangle$ has no pure Nash equilibrium where some player chooses the strategy $n+1$, it follows that $s_{1}, s_{2} \in[n]$. Hence, $\left\langle s_{1}, s_{2}\right\rangle$ is a pure Nash equilibrium for $\langle\mathrm{R}, \mathrm{C}\rangle$. A contradiction. A corresponding argument applies to yield a contradiction when $\bar{\sigma}_{2}(n+1)>0$.

By Lemmas 3.3 and 3.4 , it follows that $\mathcal{N} \mathcal{E}(\langle\bar{R}, \bar{C}\rangle)=\mathcal{N} \mathcal{E}(\langle\mathrm{R}, \mathrm{C}\rangle)$, which completes the proof of Condition (C.2). 
Since polynomial time suffices for deciding the existence of and computing a pure Nash equilibrium for a bimatrix game, Proposition 3.1 implies that computing a Nash equilibrium for a win-lose bimatrix game with the positive utility property is as hard as computing a Nash equilibrium for a win-lose bimatrix game. Hence, computing a Nash equilibrium for a win-lose bimatrix game with the positive utility property is $\mathcal{P} \mathcal{P} \mathcal{A D}$-hard. (The $\mathcal{P} \mathcal{P} \mathcal{A D}$-hardness will be extended later to symmetric win-lose bimatrix games with the positive utility property.)

\section{Win-Lose Gadgets}

Here we collect together the win-lose games that will be used as gadgets in later proofs.

\subsection{The Cyclic Game $\widehat{G}_{1}[h]$}

For an integer $h \geq 1$, define the win-lose bimatrix game $\widehat{G}_{1}[h]:=\left\langle[2],\left\{\widehat{\Sigma}_{i}\right\}_{i \in[2]},\left\{\widehat{U}_{i}\right\}_{i \in[2]}\right\rangle$ with $\widehat{\Sigma}_{1}=\widehat{\Sigma}_{2}=\left\{s_{0}, \ldots, s_{h-1}\right\} ; \widehat{U}_{1}(\mathbf{s})=1$ if and only if $\mathbf{s}=\left\langle s_{i}, s_{i}\right\rangle$ and $\widehat{U}_{2}(\mathbf{s})=1$ if and only if $\mathbf{s}=\left\langle s_{i}, s_{i+1}\right\rangle$ (with addition taken modulo $h$ ). So, roughly speaking, player 1 wins if and only if the two players concur, while player 2 wins if and only if the two players choose successive strategies with player 2 following. Clearly, $\widehat{G}_{1}[h]$ has the positive utility property. We prove:

Proposition $4.1 \widehat{\mathrm{G}}_{1}[h]$ has a single Nash equilibrium $\boldsymbol{\sigma}$, which is fully mixed and uniform with $\widehat{\mathrm{U}}_{1}(\boldsymbol{\sigma})=\widehat{\mathrm{U}}_{2}(\boldsymbol{\sigma})=\frac{1}{h} ;$ for $h=1, \boldsymbol{\sigma}$ is Pareto-Optimal and Strongly Pareto-Optimal.

Proof: Fix a Nash equilibrium $\boldsymbol{\sigma}$. We first prove that $\boldsymbol{\sigma}$ is fully mixed. Consider first player 1 and a strategy $s \in \widehat{\Sigma}_{1}$ with $\sigma_{1}(s)>0$. Then, by Lemma 2.1 (Condition (1)), it follows that $\widehat{\mathrm{U}}_{1}(\boldsymbol{\sigma})=\widehat{\mathrm{U}}_{1}\left(\boldsymbol{\sigma}_{-1} \diamond s\right)$. Assume, by way of contradiction, that $\sigma_{1}(s-1)=0$. If $\sigma_{2}(s)=0$, then $\widehat{\mathrm{U}}_{1}\left(\boldsymbol{\sigma}_{-1} \diamond s\right)=0$, so that $\widehat{\mathrm{U}}_{1}(\boldsymbol{\sigma})=0$, a contradiction to Lemma 2.2. Hence, $\sigma_{2}(s)>0$. By Lemma 2.1 (Condition (1)), it follows that $\widehat{\mathrm{U}}_{2}(\boldsymbol{\sigma})=\widehat{\mathrm{U}}_{2}\left(\boldsymbol{\sigma}_{-2} \diamond s\right)=0$, a contradiction to Lemma 2.2, A similar argument proves that $\sigma_{2}(s)>0$ for each $s \in \widehat{\Sigma}_{2}$.

To prove that $\boldsymbol{\sigma}$ is uniform, assume, by way of contradiction, that there are indices $j, k \in \widehat{\Sigma}_{1}$ with $\sigma_{1}(j) \neq \sigma_{1}(k)$. Since $\operatorname{Supp}\left(\sigma_{2}\right)=\widehat{\Sigma}_{2}$, it follows, by Lemma 2.1 (Condition (1)), that $\widehat{\mathrm{U}}_{2}(\boldsymbol{\sigma})=\widehat{\mathrm{U}}_{2}\left(\boldsymbol{\sigma}_{-2} \diamond(j+1)\right)=\widehat{\mathrm{U}}_{2}\left(\boldsymbol{\sigma}_{-2} \diamond(k+1)\right)$, so that $\sigma_{1}(j)=\sigma_{1}(k)$, a contradiction. A similar argument proves that for all indices $j, k \in \widehat{\Sigma}_{2}, \sigma_{2}(j)=\sigma_{2}(k)$, and this implies that there is a single Nash equilibrium. Thus, $\widehat{\mathrm{U}}_{1}(\boldsymbol{\sigma})=\widehat{\mathrm{U}}_{2}(\boldsymbol{\sigma})=1 \cdot h \cdot \frac{1}{h} \cdot \frac{1}{h}=\frac{1}{h}$. For $h=1,\left|\widehat{\Sigma}_{1}\right|=\left|\widehat{\Sigma}_{2}\right|=1$ and $\boldsymbol{\sigma}$ is pure; clearly, $\boldsymbol{\sigma}$ is also both Pareto-Optimal and Strongly Pareto-Optimal.

By Proposition 4.1, the win-lose game $\widehat{G}_{1}[h]$ is a negative instance for $\exists$ UNIFORM NASH; when $h=1$, it is also a negative instance for $\exists \neg$ PARETO-OPTIMAL NASH and for $\exists \neg$ STRONGLY PARETO-OPTIMAL NASH. 


\subsection{The Irrational Game $\widehat{G}_{2}$}

Define the win-lose bimatrix game $\widehat{G}_{2}=\left\langle[3],\left\{\widehat{\Sigma}_{i}\right\}_{i \in[3]},\left\{\widehat{U}_{i}\right\}_{i \in[3]}\right\rangle$ with $\widehat{\Sigma}_{1}=\widehat{\Sigma}_{2}=\{0,1\}$ and $\widehat{\Sigma}_{3}=\{0,1,2\}$; the utility functions are depicted below:

\begin{tabular}{||c|c||c|c||c|c||}
\hline Profile s & Utility vector $\widehat{\mathrm{U}}(\mathbf{s})$ & Profile $\mathbf{s}$ & Utility vector $\widehat{\mathrm{U}}(\mathbf{s})$ & Profile $\mathbf{s}$ & Utility vector $\widehat{\mathrm{U}}(\mathbf{s})$ \\
\hline \hline$\langle 0,0,0\rangle$ & $\langle 1,0,1\rangle$ & $\langle 0,1,1\rangle$ & $\langle 1,0,0\rangle$ & $\langle 1,0,2\rangle$ & $\langle 1,0,0\rangle$ \\
\hline$\langle 0,0,1\rangle$ & $\langle 1,1,0\rangle$ & $\langle 0,1,2\rangle$ & $\langle 0,0,1\rangle$ & $\langle 1,1,0\rangle$ & $\langle 1,1,0\rangle$ \\
\hline$\langle 0,0,2\rangle$ & $\langle 0,1,0\rangle$ & $\langle 1,0,0\rangle$ & $\langle 0,0,1\rangle$ & $\langle 1,1,1\rangle$ & $\langle 0,0,1\rangle$ \\
\hline$\langle 0,1,0\rangle$ & $\langle 0,1,0\rangle$ & $\langle 1,0,1\rangle$ & $\langle 0,1,1\rangle$ & $\langle 1,1,2\rangle$ & $\langle 1,1,0\rangle$ \\
\hline \hline
\end{tabular}

Clearly, $\widehat{G}_{2}$ has the positive utility property. We prove:

Proposition $4.2 \widehat{\mathrm{G}}_{2}$ has a single Nash equilibrium, which is irrational.

Proof: The following table establishes that $\widehat{\mathrm{G}}_{2}$ has no pure Nash equilibrium:

\begin{tabular}{||c|c|c||c|c|c||}
\hline Profile s & Player: & Improves by switching to: & Profile s & Player: & Improves by switching to: \\
\hline \hline$\langle 0,0,0\rangle$ & 2 & 1 & $\langle 1,0,0\rangle$ & 1 & 0 \\
\hline$\langle 0,0,1\rangle$ & 3 & 0 & $\langle 1,0,1\rangle$ & 1 & 0 \\
\hline$\langle 0,0,2\rangle$ & 1 & 1 & $\langle 1,0,2\rangle$ & 3 & 1 \\
\hline$\langle 0,1,0\rangle$ & 3 & 2 & $\langle 1,1,0\rangle$ & 3 & 1 \\
\hline$\langle 0,1,1\rangle$ & 3 & 2 & $\langle 1,1,1\rangle$ & 1 & 0 \\
\hline$\langle 0,1,2\rangle$ & 1 & 1 & $\langle 1,1,2\rangle$ & 3 & 1 \\
\hline \hline
\end{tabular}

We now turn to a mixed Nash equilibrium $\boldsymbol{\sigma}$, which shall be represented with the vector of probabilities $\left\langle\sigma_{1}, \sigma_{2}, \sigma_{3}^{0}, \sigma_{3}^{1}\right\rangle$, where for each player $i \in[2], \sigma_{i}:=\sigma_{i}(0)$, while $\sigma_{3}^{s}:=\sigma_{3}(s)$ for $s \in\{0,1\}$; thus, for each player $i \in[2], \sigma_{i}(1)=1-\sigma_{i}$, while $\sigma_{3}(2)=1-\sigma_{3}^{0}-\sigma_{3}^{1}$. We calculate 
the conditional expected utilities $\widehat{\mathrm{U}}_{i}\left(\mathbf{s}_{-i} \diamond s\right)$ for each player $i \in[3]$ and strategy $s \in \widehat{\Sigma}_{i}$ :

$$
\begin{aligned}
\widehat{\mathrm{U}}_{1}\left(\boldsymbol{\sigma}_{-1} \diamond 0\right) & =\sigma_{2} \cdot \sigma_{3}^{0}+\sigma_{2} \cdot \sigma_{3}^{1}+\left(1-\sigma_{2}\right) \cdot \sigma_{3}^{1} \\
& =\sigma_{2} \cdot \sigma_{3}^{0}+\sigma_{3}^{1}, \\
\widehat{\mathrm{U}}_{1}\left(\boldsymbol{\sigma}_{-1} \diamond 1\right) & =\sigma_{2} \cdot\left(1-\sigma_{3}^{0}-\sigma_{3}^{1}\right)+\left(1-\sigma_{2}\right) \cdot \sigma_{3}^{0}+\left(1-\sigma_{2}\right) \cdot\left(1-\sigma_{3}^{0}-\sigma_{3}^{1}\right) \\
& =1-\sigma_{3}^{1}-\sigma_{2} \cdot \sigma_{3}^{0}, \\
\widehat{\mathrm{U}}_{2}\left(\boldsymbol{\sigma}_{-2} \diamond 0\right) & =\sigma_{1} \cdot \sigma_{3}^{1}+\sigma_{1} \cdot\left(1-\sigma_{3}^{0}-\sigma_{3}^{1}\right)+\left(1-\sigma_{1}\right) \cdot \sigma_{3}^{1} \\
& =\sigma_{1} \cdot\left(1-\sigma_{3}^{0}-\sigma_{3}^{1}\right)+\sigma_{3}^{1}, \\
\widehat{\mathrm{U}}_{2}\left(\boldsymbol{\sigma}_{-2} \diamond 1\right) & =\sigma_{1} \cdot \sigma_{3}^{0}+\left(1-\sigma_{1}\right) \cdot \sigma_{3}^{0}+\left(1-\sigma_{1}\right) \cdot\left(1-\sigma_{3}^{0}-\sigma_{3}^{1}\right) \\
\widehat{\mathrm{U}}_{3}\left(\boldsymbol{\sigma}_{-3} \diamond 0\right) & =\sigma_{3}^{0}+\left(1-\sigma_{1}\right) \cdot\left(1-\sigma_{3}^{0}-\sigma_{3}^{1}\right), \\
\widehat{\mathrm{U}}_{3}\left(\boldsymbol{\sigma}_{-3} \diamond 1\right) & =\left(1-\sigma_{2}+\left(1-\sigma_{1}\right) \cdot \sigma_{2}\right. \\
\widehat{\mathrm{U}}_{3}\left(\boldsymbol{\sigma}_{-3} \diamond 2\right) & =\sigma_{2},\left(1-\sigma_{1}\right) \cdot\left(1-\sigma_{2}\right) \\
& =1-\sigma_{1},
\end{aligned}
$$

We now examine all possible cases for $\boldsymbol{\sigma}$. We shall derive a contradiction for all but one case, where we shall establish that $\sigma$ is irrational.

- Only player 1 is mixed. Then, $\sigma_{2} \in\{0,1\}$ and $\left(\sigma_{3}^{0}, \sigma_{3}^{1}\right) \in\{(0,0),(0,1),(1,0)\}$. Since $\boldsymbol{\sigma}$ is a Nash equilibrium where player 1 is mixed, Lemma 2.1 (Condition (1)) implies that $\widehat{\mathrm{U}}_{1}\left(\boldsymbol{\sigma}_{-1} \diamond 0\right)=\widehat{\mathrm{U}}_{1}\left(\boldsymbol{\sigma}_{-1} \diamond 1\right)$, or $\sigma_{2} \cdot \sigma_{3}^{0}+\sigma_{3}^{1}=1-\sigma_{3}^{1}-\sigma_{2} \cdot \sigma_{3}^{0}$, or $2 \cdot\left(\sigma_{2} \cdot \sigma_{3}^{0}+\sigma_{3}^{1}\right)=1$. Hence, for $\sigma_{2}=0$ (resp., $\left.\sigma_{2}=1\right), \sigma_{3}^{1}=\frac{1}{2}$ (resp., $\sigma_{3}^{0}+\sigma_{3}^{1}=\frac{1}{2}$ ). A contradiction.

- Only player 2 is mixed. Then, $\sigma_{1} \in\{0,1\}$ and $\left(\sigma_{3}^{0}, \sigma_{3}^{1}\right) \in\{(0,0),(0,1),(1,0)\}$. Since $\boldsymbol{\sigma}$ is a Nash equilibrium where player 2 is mixed, Lemma 2.1 (Condition (1)) implies that $\widehat{\mathrm{U}}_{2}\left(\boldsymbol{\sigma}_{-2} \diamond 0\right)=\widehat{\mathrm{U}}_{2}\left(\boldsymbol{\sigma}_{-2} \diamond 1\right)$, or $\left(2 \sigma_{1}-1\right) \cdot\left(1-\sigma_{3}^{0}-\sigma_{3}^{1}\right)=\sigma_{3}^{0}-\sigma_{3}^{1}$. Hence, for $\sigma_{1}=0$ (resp., $\sigma_{1}=1$ ), $1-\sigma_{3}^{0}-\sigma_{3}^{1}=\sigma_{3}^{1}-\sigma_{3}^{0}$ (resp., $1-\sigma_{3}^{0}-\sigma_{3}^{1}=\sigma_{3}^{0}-\sigma_{3}^{1}$ ), or $\sigma_{3}^{1}=\frac{1}{2}$ (resp., $\left.\sigma_{3}^{0}=\frac{1}{2}\right)$. A contradiction.

- Only player 3 is mixed. Then, $\left(\sigma_{1}, \sigma_{2}\right) \in\{(0,0),(0,1),(1,0),(1,1)\}$. Since $\boldsymbol{\sigma}$ is a Nash equilibrium where player 3 is mixed, Lemma 2.1 (Condition (1)) implies that there are strategies $s, t \in\{0,1,2\}$ such that $\widehat{\mathrm{U}}_{3}\left(\boldsymbol{\sigma}_{-3} \diamond s\right)=\widehat{\mathrm{U}}_{3}\left(\boldsymbol{\sigma}_{-3} \diamond t\right)$. By Lemma 2.2. it follows that $\widehat{\mathrm{U}}_{3}\left(\boldsymbol{\sigma}_{-3} \diamond s\right)=\widehat{\mathrm{U}}_{3}\left(\boldsymbol{\sigma}_{-3} \diamond t\right)>0$. Inspecting the following table yields that $\sigma_{1}=0$, $\sigma_{2}=1$ and $\sigma_{3}^{2}=0$ : 


\begin{tabular}{||c|c|c|c|c||}
\hline \hline$\sigma_{1}$ & $\sigma_{2}$ & $\widehat{\mathrm{U}}_{3}\left(\boldsymbol{\sigma}_{-3} \diamond 0\right)=\sigma_{2}$ & $\widehat{\mathrm{U}}_{3}\left(\boldsymbol{\sigma}_{-3} \diamond 1\right)=1-\sigma_{1}$ & $\widehat{\mathrm{U}}_{3}\left(\boldsymbol{\sigma}_{-3} \diamond 2\right)=\sigma_{1} \cdot\left(1-\sigma_{2}\right)$ \\
\hline \hline 0 & 0 & 0 & 1 & 0 \\
\hline 0 & 1 & 1 & 1 & 0 \\
\hline 1 & 0 & 0 & 0 & 0 \\
\hline 1 & 1 & 1 & 0 & 0 \\
\hline \hline
\end{tabular}

Hence, $\sigma_{3}^{0}+\sigma_{3}^{1}=1$. Since $\boldsymbol{\sigma}$ is a Nash equilibrium and $\sigma_{1}=0$, Lemma 2.1 (Condition (2)) implies that $\widehat{U}_{1}\left(\boldsymbol{\sigma}_{-1} \diamond 1\right) \geq \widehat{\mathbf{U}}_{1}\left(\boldsymbol{\sigma}_{-1} \diamond 0\right)$. Since $\sigma_{2}=1$, it follows that $1-\sigma_{3}^{1}-\sigma_{3}^{0} \geq \sigma_{3}^{0}+\sigma_{3}^{1}$, or $\sigma_{3}^{0}+\sigma_{3}^{1} \leq \frac{1}{2}$. A contradiction.

- Only players 2 and 3 are mixed. Since player 1 is pure, $\sigma_{1} \in\{0,1\}$. Assume, by way of contradiction, that $\sigma_{1}=0$. Then, $\widehat{\mathrm{U}}_{3}\left(\boldsymbol{\sigma}_{-3} \diamond 0\right)=\sigma_{2}, \widehat{\mathrm{U}}_{3}\left(\boldsymbol{\sigma}_{-1} \diamond 1\right)=1$ and $\widehat{\mathrm{U}}_{3}\left(\boldsymbol{\sigma}_{-1} \diamond 2\right)=$ 0 . Since $\boldsymbol{\sigma}$ is a Nash equilibrium, Lemma 2.1 (Condition (2)) implies that $2 \notin \operatorname{Supp}\left(\sigma_{3}\right)$. Since $\boldsymbol{\sigma}$ is a Nash equilibrium where player 3 is mixed, Lemma 2.1 (Condition (1)) implies that $\widehat{\mathrm{U}}_{3}\left(\boldsymbol{\sigma}_{-3} \diamond 0\right)=\widehat{\mathrm{U}}_{3}\left(\boldsymbol{\sigma}_{-1} \diamond 1\right)$, which implies that $\sigma_{2}=1$, so that player 2 is pure. A contradiction. It follows that $\sigma_{1}=1$. Then, $\widehat{\mathrm{U}}_{2}\left(\boldsymbol{\sigma}_{-2} \diamond 0\right)=1-\sigma_{3}^{0}$ and $\widehat{\mathrm{U}}_{2}\left(\boldsymbol{\sigma}_{-2} \diamond 1\right)=\sigma_{3}^{0}$.

Since $\boldsymbol{\sigma}$ is a Nash equilibrium where player 2 is mixed, Lemma 2.1 (Condition (1)) implies that $\widehat{\mathrm{U}}_{2}\left(\boldsymbol{\sigma}_{-2} \diamond 0\right)=\widehat{\mathrm{U}}_{2}\left(\boldsymbol{\sigma}_{-2} \diamond 1\right)$, or $\sigma_{3}^{0}=\frac{1}{2}$. Also, $\widehat{\mathrm{U}}_{3}\left(\boldsymbol{\sigma}_{-3} \diamond 0\right)=\sigma_{2}, \widehat{\mathrm{U}}_{3}\left(\boldsymbol{\sigma}_{-3} \diamond 1\right)=$ 0 and $\widehat{\mathrm{U}}_{3}\left(\boldsymbol{\sigma}_{-3} \diamond 2\right)=1-\sigma_{2}$. Since player 2 is mixed, $\sigma_{2}>0$. Since $\boldsymbol{\sigma}$ is a Nash equilibrium, Lemma 2.1 (Condition (2)) implies that $1 \notin \operatorname{Supp}\left(\sigma_{3}\right)$, or $\sigma_{3}^{1}=0$. Since $\boldsymbol{\sigma}$ is a Nash equilibrium where player 3 is mixed, Lemma 2.1 (Condition (1)) implies that $\widehat{\mathrm{U}}_{3}\left(\boldsymbol{\sigma}_{-3} \diamond 0\right)=\widehat{\mathrm{U}}_{3}\left(\boldsymbol{\sigma}_{-3} \diamond 2\right)$. It follows that $\sigma_{2}=\frac{1}{2}$.

Since $\sigma_{1}=1$ and $\sigma$ is a Nash equilibrium, Lemma 2.1 (Condition (2)) implies that $\widehat{\mathrm{U}}_{1}\left(\boldsymbol{\sigma}_{-1} \diamond 0\right) \geq \widehat{\mathrm{U}}_{1}\left(\boldsymbol{\sigma}_{-1} \diamond 1\right)$, or $\sigma_{2} \cdot \sigma_{3}^{0}+\sigma_{3}^{1} \geq 1-\sigma_{3}^{1}-\sigma_{2} \cdot \sigma_{3}^{1}$. Hence, $\frac{1}{2} \cdot \frac{1}{2}+0 \geq 1-0-\frac{1}{2} \cdot \frac{1}{2}$. A contradiction.

- Only players 1 and 3 are mixed. Since player 2 is pure, $\sigma_{2} \in\{0,1\}$. Assume, by way of contradiction, that $\sigma_{2}=1$. Then, $\widehat{\mathrm{U}}_{3}\left(\boldsymbol{\sigma}_{-3} \diamond 0\right)=1, \widehat{\mathrm{U}}_{3}\left(\boldsymbol{\sigma}_{-3} \diamond 1\right)=1-\sigma_{1}$ and $\widehat{\mathrm{U}}_{3}\left(\boldsymbol{\sigma}_{-3} \diamond 2\right)=0$. Since $\boldsymbol{\sigma}$ is a Nash equilibrium, Lemma 2.1 (Condition (2)) implies that $2 \notin \operatorname{Supp}\left(\sigma_{3}\right)$, or $\sigma_{3}^{2}=0$. Since $\boldsymbol{\sigma}$ is a Nash equilibrium where player 3 is mixed, Lemma 2.1 (Condition (1)) implies that $\widehat{\mathrm{U}}_{3}\left(\boldsymbol{\sigma}_{-3} \diamond 0\right)=\widehat{\mathrm{U}}_{3}\left(\boldsymbol{\sigma}_{-3} \diamond 1\right)$, which implies that $\sigma_{1}=0$, so that player 1 is pure. A contradiction. It follows that $\sigma_{2}=0$.

Then, $\widehat{\mathrm{U}}_{3}\left(\boldsymbol{\sigma}_{-3} \diamond 0\right)=0, \widehat{\mathrm{U}}_{3}\left(\boldsymbol{\sigma}_{-3} \diamond 1\right)=1-\sigma_{1}$ and $\widehat{\mathrm{U}}_{3}\left(\boldsymbol{\sigma}_{-3} \diamond 2\right)=\sigma_{1}$. Since player 1 is mixed, $\sigma_{1}>0$. Since $\boldsymbol{\sigma}$ is a Nash equilibrium, Lemma 2.1 (Condition (2)) implies that $0 \notin \operatorname{Supp}\left(\sigma_{3}\right)$, or $\sigma_{3}(0)=0$.

Since $\boldsymbol{\sigma}$ is a Nash equilibrium where player 1 is mixed, Lemma 2.1(Condition (1)) implies that $\widehat{U}_{1}\left(\boldsymbol{\sigma}_{-1} \diamond 0\right)=\widehat{U}_{1}\left(\boldsymbol{\sigma}_{-1} \diamond 1\right)$, or $\sigma_{3}^{1}=1-\sigma_{3}^{1}$, so that $\sigma_{3}^{1}=\frac{1}{2}$. 
Since $\boldsymbol{\sigma}$ is a Nash equilibrium where player 3 is mixed, Lemma 2.1(Condition (1)) implies that $\widehat{\mathrm{U}}_{3}\left(\boldsymbol{\sigma}_{-3} \diamond 1\right)=\widehat{\mathrm{U}}_{3}\left(\boldsymbol{\sigma}_{-3} \diamond 2\right)$, or $1-\sigma_{1}=\sigma_{1}$, or $\sigma_{1}=\frac{1}{2}$. Since $\sigma_{2}=0$ and $\boldsymbol{\sigma}$ is a Nash equilibrium, Lemma 2.1 (Condition (2)) implies that $\widehat{\mathrm{U}}_{2}\left(\boldsymbol{\sigma}_{-2} \diamond 0\right) \leq \widehat{\mathrm{U}}_{2}\left(\boldsymbol{\sigma}_{-2} \diamond 1\right)$, or $\frac{1}{2} \cdot\left(1-0-\frac{1}{2}\right)+\frac{1}{2} \leq 0+\frac{1}{2} \cdot\left(1-0-\frac{1}{2}\right)$ or $\frac{1}{4} \leq-\frac{1}{4}$. A contradiction.

- Only players 1 and 2 are mixed. Since player 3 is pure, $\left(\sigma_{3}^{0}, \sigma_{3}^{1}\right) \in\{(0,0),(0,1),(1,0)\}$. Since $\sigma$ is a Nash equilibrium where player 1 is mixed, Lemma 2.1 (Condition (1)) implies that $\widehat{\mathrm{U}}_{1}\left(\boldsymbol{\sigma}_{-1} \diamond 0\right)=\widehat{\mathrm{U}}_{1}\left(\boldsymbol{\sigma}_{-1} \diamond 1\right)$, or $\sigma_{2} \cdot \sigma_{3}^{0}+\sigma_{3}^{1}=1-\sigma_{3}^{1}-\sigma_{2} \cdot \sigma_{3}^{0}$ or $2 \cdot\left(\sigma_{2} \cdot \sigma_{3}^{0}+\sigma_{3}^{1}\right)=1$. This implies that $\left(\sigma_{3}^{0}, \sigma_{3}^{1}\right) \notin\{(0,0),(0,1)\}$; so, $\left(\sigma_{3}^{0}, \sigma_{3}^{1}\right)=(1,0)$.

Since $\boldsymbol{\sigma}$ is a Nash equilibrium where player 2 is mixed, Lemma 2.1(Condition (1)) implies that $\widehat{\mathrm{U}}_{2}\left(\boldsymbol{\sigma}_{-2} \diamond 0\right)=\widehat{\mathrm{U}}_{2}\left(\boldsymbol{\sigma}_{-2} \diamond 1\right)$, or $\sigma_{1} \cdot\left(1-\sigma_{3}^{0}-\sigma_{3}^{1}\right)+\sigma_{3}^{1}=\sigma_{3}^{0}+\left(1-\sigma_{1}\right) \cdot\left(1-\sigma_{3}^{0}-\sigma_{3}^{1}\right)$ or $\sigma_{1} \cdot 0+0=1+\left(1-\sigma_{1}\right) \cdot 0$ or $0=1$. A contradiction.

- All players are mixed. We proceed by case analysis. Assume first that $\sigma_{3}^{0}=0$. Since $\boldsymbol{\sigma}$ is a Nash equilibrium where player 1 is mixed, Lemma 2.1 (Condition (1)) implies that $\widehat{\mathrm{U}}_{1}\left(\boldsymbol{\sigma}_{-1} \diamond 0\right)=\widehat{\mathrm{U}}_{1}\left(\boldsymbol{\sigma}_{-1} \diamond 1\right)$, or $\sigma_{3}^{1}=1-\sigma_{3}^{1}$, so that $\sigma_{3}^{1}=\frac{1}{2}$.

Since $\sigma$ is a Nash equilibrium where player 2 is mixed, Lemma 2.1 (Condition (1)) implies that $\widehat{\mathrm{U}}_{2}\left(\boldsymbol{\sigma}_{-2} \diamond 0\right)=\widehat{\mathrm{U}}_{2}\left(\boldsymbol{\sigma}_{-2} \diamond 1\right)$, or $\sigma_{1} \cdot \frac{1}{2}+\frac{1}{2}=\left(1-\sigma_{1}\right) \cdot \frac{1}{2}$ or $\sigma_{1}=0$, so that player 1 is pure. A contradiction.

Assume now that $\sigma_{3}^{1}=0$. Since $\boldsymbol{\sigma}$ is a Nash equilibrium where player 3 is mixed, Lemma 2.1 (Condition (1)) implies that $\widehat{\mathrm{U}}_{3}\left(\boldsymbol{\sigma}_{-3} \diamond 0\right)=\widehat{\mathrm{U}}_{3}\left(\boldsymbol{\sigma}_{-3} \diamond 2\right)$, or $\sigma_{2}=\sigma_{1} \cdot\left(1-\sigma_{2}\right)$. Since $\boldsymbol{\sigma}$ is a Nash equilibrium where player 1 is mixed, Lemma 2.1 (Condition (1)) implies that $\widehat{\mathrm{U}}_{1}\left(\boldsymbol{\sigma}_{-1} \diamond 0\right)=\widehat{\mathrm{U}}_{1}\left(\boldsymbol{\sigma}_{-1} \diamond 1\right)$, or $\sigma_{2} \cdot \sigma_{3}^{0}=1-\sigma_{2} \cdot \sigma_{3}^{0}$ so that $2 \sigma_{2} \cdot \sigma_{3}^{0}=1$. Since $\boldsymbol{\sigma}$ is a Nash equilibrium where player 2 is mixed, Lemma 2.1 (Condition (1)) implies that $\widehat{\mathrm{U}}_{2}\left(\boldsymbol{\sigma}_{-2} \diamond 0\right)=\widehat{\mathrm{U}}_{2}\left(\boldsymbol{\sigma}_{-2} \diamond 1\right)$, or $\sigma_{1} \cdot\left(1-\sigma_{3}^{0}\right)=\sigma_{3}^{0}+\left(1-\sigma_{1}\right) \cdot\left(1-\sigma_{3}^{0}\right)$ or $2 \sigma_{1}-1=\frac{\sigma_{3}^{0}}{1-\sigma_{3}^{0}}$. Hence, it follows that $\left(2 \sigma_{1}-1\right) \cdot\left(2 \sigma_{2}-1\right)=1$. Thus, $\left(2 \frac{\sigma_{2}}{\sigma_{2}-1}-1\right) \cdot\left(2 \sigma_{2}-1\right)=1$, or $\left(-1+3 \sigma_{2}\right) \cdot\left(2 \sigma_{2}-1\right)=1-\sigma_{2}$, which yields $\sigma_{2}=\frac{2}{3}$. Hence, $\sigma_{1}=\frac{\sigma_{2}}{1-\sigma_{2}}=2 . \mathrm{A}$ contradiction.

Assume finally that $\sigma_{3}^{2}=0$, so that $\sigma_{3}^{0}+\sigma_{3}^{1}=1$. Since $\boldsymbol{\sigma}$ is a Nash equilibrium where player 1 is mixed, Lemma 2.1 (Condition (1)) implies that $\widehat{U}_{1}\left(\boldsymbol{\sigma}_{-1} \diamond 0\right)=\widehat{U}_{1}\left(\boldsymbol{\sigma}_{-1} \diamond 1\right)$, or $\sigma_{2} \cdot \sigma_{3}^{0}+\sigma_{3}^{1}=1-\sigma_{3}^{1}-\sigma_{2} \cdot \sigma_{3}^{0}$ or $2 \sigma_{2} \sigma_{3}^{0}+2 \cdot\left(1-\sigma_{3}^{0}\right)=1$, so that $2 \sigma_{3}^{0} \cdot\left(1-\sigma_{2}\right)=1$. Since $\boldsymbol{\sigma}$ is a Nash equilibrium where player 2 is mixed, Lemma 2.1 (Condition (1)) implies that $\widehat{\mathrm{U}}_{2}\left(\boldsymbol{\sigma}_{-2} \diamond 0\right)=\widehat{\mathrm{U}}_{2}\left(\boldsymbol{\sigma}_{-2} \diamond 1\right)$, or $\sigma_{3}^{1}=\sigma_{3}^{0}$. It follows that $\sigma_{3}^{0}=\frac{1}{2}$, so that $\sigma_{2}=0$ and player 2 is pure. A contradiction. It follows from the case analysis that $0<\sigma_{3}^{0}, \sigma_{3}^{1}, \sigma_{3}^{2}<1$, and player 3 is fully mixed.

Since $\boldsymbol{\sigma}$ is a Nash equilibrium where player 3 is fully mixed, Lemma 2.1 (Condition 
(1)) implies that $\widehat{\mathrm{U}}_{3}\left(\boldsymbol{\sigma}_{-3} \diamond 0\right)=\widehat{\mathrm{U}}_{3}\left(\boldsymbol{\sigma}_{-3} \diamond 1\right)=\widehat{\mathrm{U}}_{3}\left(\boldsymbol{\sigma}_{-3} \diamond 2\right)$, or $\sigma_{2}=1-\sigma_{1}=\sigma_{1}$. $\left(1-\sigma_{2}\right)$. Hence, $\left(1-\sigma_{2}\right)^{2}=\sigma_{2}$, so that $\sigma_{2}=\frac{3-\sqrt{5}}{2}$ and $\sigma_{1}=\frac{\sqrt{5}-1}{2}$. Since $\sigma$ is a Nash equilibrium where player 1 is mixed, Lemma 2.1 (Condition (1)) implies that $\widehat{\mathrm{U}}_{1}\left(\boldsymbol{\sigma}_{-1} \diamond 0\right)=\widehat{\mathrm{U}}_{1}\left(\boldsymbol{\sigma}_{-1} \diamond 1\right)$, or $2 \sigma_{2} \cdot \sigma_{3}^{0}+2 \sigma_{3}^{1}=1$. Since $\boldsymbol{\sigma}$ is a Nash equilibrium where player 2 is mixed, Lemma 2.1 (Condition (1)) implies that $\widehat{U}_{2}\left(\boldsymbol{\sigma}_{-2} \diamond 0\right)=\widehat{\mathrm{U}}_{2}\left(\boldsymbol{\sigma}_{-2} \diamond 1\right)$, or $\left(2 \sigma_{1}-1\right) \cdot\left(1-\sigma_{3}^{0}-\sigma_{3}^{1}\right)=\sigma_{3}^{0}-\sigma_{3}^{1}$ or $2\left(1-\sigma_{1}\right) \cdot \sigma_{3}^{1}-2 \sigma_{1} \cdot \sigma_{3}^{0}=1-2 \sigma_{1}$. Combining we obtain $\left(2 \sigma_{1}+2\left(1-\sigma_{1}\right) \cdot \sigma_{2}\right) \cdot \sigma_{3}^{1}=\left(1-2 \sigma_{1}\right) \cdot \sigma_{2}+\sigma_{1}$, which yields $\sigma_{3}^{1}=\frac{5-\sqrt{5}}{8}$. Now, $\sigma_{3}^{0}=\frac{1-2 \sigma_{3}^{1}}{2 \sigma_{2}}=\frac{1+\sqrt{5}}{8}$, and $\sigma_{3}^{2}=1-\sigma_{3}^{0}-\sigma_{3}^{1}=\frac{1}{4}$.

The claim now follows.

By Proposition 4.2, the win-lose 3-player game $\widehat{G}_{2}$ is a negative instance for $\exists$ RATIONAL NASH. Although $\widehat{G}_{2}$ is also a negative instance for $\exists$ UNIFORM NASH and for $\exists$ SYMMETRIC NASH we shall present next the win-lose bimatrix games $\widehat{G}_{3}$ and $\widehat{G}_{4}$ as negative instances for $\exists$ UNIFORM NASH and for $\exists$ SYMMETRIC NASH, respectively, since we seek to establish that these two decision problems are $\mathcal{N} \mathcal{P}$-hard for win-lose bimatrix games.

\subsection{The Non-Uniform Game $\widehat{G}_{3}$}

Define the win-lose game $\widehat{\mathrm{G}}_{3}=\left\langle[2],\left\{\widehat{\Sigma}_{i}\right\}_{i \in[2]},\left\{\widehat{\mathrm{U}}_{i}\right\}_{i \in[2]}\right\rangle$ with $\widehat{\Sigma}_{1}=\widehat{\Sigma}_{2}=[4]$; the utility functions are depicted below:

\begin{tabular}{||c|c||c|c||c|c||c|c||}
\hline Profile $\mathbf{s}$ & $\widehat{\mathrm{U}}(\mathbf{s})$ & Profile $\mathbf{s}$ & $\widehat{\mathrm{U}}(\mathbf{s})$ & Profile s & $\widehat{\mathrm{U}}(\mathbf{s})$ & Profile s & $\widehat{\mathrm{U}}(\mathbf{s})$ \\
\hline \hline$\langle 1,1\rangle$ & $\langle 0,1\rangle$ & $\langle 1,2\rangle$ & $\langle 0,1\rangle$ & $\langle 1,3\rangle$ & $\langle 1,0\rangle$ & $\langle 1,4\rangle$ & $\langle 1,0\rangle$ \\
\hline$\langle 2,1\rangle$ & $\langle 0,1\rangle$ & $\langle 2,2\rangle$ & $\langle 1,0\rangle$ & $\langle 2,3\rangle$ & $\langle 1,0\rangle$ & $\langle 2,4\rangle$ & $\langle 0,1\rangle$ \\
\hline$\langle 3,1\rangle$ & $\langle 0,1\rangle$ & $\langle 3,2\rangle$ & $\langle 1,0\rangle$ & $\langle 3,3\rangle$ & $\langle 0,1\rangle$ & $\langle 3,4\rangle$ & $\langle 1,0\rangle$ \\
\hline$\langle 4,1\rangle$ & $\langle 1,0\rangle$ & $\langle 4,2\rangle$ & $\langle 0,1\rangle$ & $\langle 4,3\rangle$ & $\langle 0,1\rangle$ & $\langle 4,4\rangle$ & $\langle 0,1\rangle$ \\
\hline \hline
\end{tabular}

Clearly, $\widehat{G}_{3}$ has the positive utility property. Note that for each profile s, exactly one of $\widehat{U}_{1}(\mathbf{s})=$ 1 and $\widehat{\mathrm{U}}_{2}(\mathbf{s})=1$ holds, and $\widehat{\mathrm{G}}_{3}$ is 1 -sum. Define the shape of a profile $\mathbf{s}$ to be square (resp., circle) when $\widehat{\mathrm{U}}_{1}(\mathbf{s})=1$ (resp., $\widehat{\mathrm{U}}_{2}(\mathbf{s})=1$ ). Figure 1 presents a bimatricial representation of the set of profiles for $\widehat{G}_{3}$, where each profile is drawn as either a square or a circle; rows and columns correspond to strategies of player 1 and 2 , respectively.

Note that in a uniform Nash equilibrium $\boldsymbol{\sigma}$, a best-response of player 2 (resp., player 1) to $\sigma_{1}$ (resp., $\sigma_{2}$ ) is a strategy $s \in \widehat{\Sigma}_{2}$ (resp., $s \in \widehat{\Sigma}_{1}$ ) whose corresponding column (resp., row), restricted to the rows (resp., the columns) corresponding to the strategies in $\operatorname{Supp}\left(\sigma_{1}\right)$ (resp., in Supp $\left(\sigma_{2}\right)$ ), contains a maximum number of circles (resp., squares). In particular, all columns 


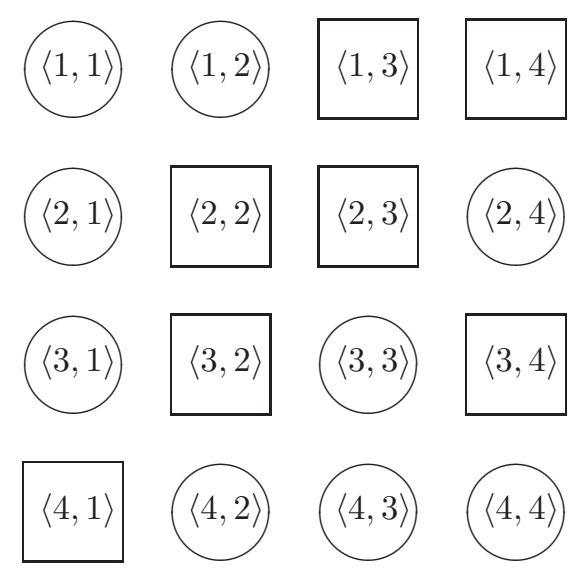

Figure 1: A bimatricial representation of all profiles in $\widehat{\mathrm{G}}_{3}$ as shapes.

(resp., rows) corresponding to strategies that are best-responses of player 2 (resp., player 1) to $\sigma_{1}$ (resp., $\sigma_{2}$ ), restricted to the rows (resp., the columns) corresponding to the strategies in $\operatorname{Supp}\left(\sigma_{1}\right)$ (resp., in Supp $\left(\sigma_{2}\right)$ ), contain the same number of circles (resp., squares). We prove:

Proposition $4.3 \widehat{\mathrm{G}}_{3}$ has no uniform Nash equilibrium.

Proof: Note first that $\widehat{G}_{3}$ has no pure Nash equilibrium. We now prove:

Lemma 4.4 (No Pure Player) Fix a Nash equilibrium $\boldsymbol{\sigma}$. Then, for each player $i \in[2]$, $\left|\operatorname{Supp}\left(\sigma_{i}\right)\right| \geq 2$.

Proof: Assume, by way of contradiction, that there is a player $i \in[2]$ with $\left|\operatorname{Supp}\left(\sigma_{i}\right)\right|=1$. Since $\boldsymbol{\sigma}$ is mixed, it follows that player $\bar{i} \in[2] \backslash\{i\}$ is mixed. Since $\boldsymbol{\sigma}$ is a Nash equilibrium where player $\bar{i}$ is mixed, Lemma2.1 (Condition (1)) implies that for each strategy $s \in \operatorname{Supp}\left(\sigma_{\bar{i}}\right)$, $\widehat{\mathrm{U}}_{\bar{i}}(\boldsymbol{\sigma})=\widehat{\mathrm{U}}_{\bar{i}}\left(\boldsymbol{\sigma}_{-\bar{i}} \diamond s\right)$. So, all profiles supported in $\boldsymbol{\sigma}_{-\bar{i}} \diamond s$ have the same shape. Since $\widehat{\mathrm{G}}_{3}$ is a 1-sum, win-lose game, either $\widehat{\mathrm{U}}_{i}(\boldsymbol{\sigma})=0$ or $\widehat{\mathrm{U}}_{\bar{i}}(\boldsymbol{\sigma})=0$. Since $\widehat{\mathrm{G}}_{3}$ has the positive utility property, Lemma 2.2 implies that both $\widehat{U}_{i}(\boldsymbol{\sigma})>0$ and $\widehat{U}_{\bar{i}}(\boldsymbol{\sigma})>0$. A contradiction.

To complete the proof, we shall establish that there is no uniform Nash equilibrium $\boldsymbol{\sigma}$ with $2 \leq\left|\operatorname{Supp}\left(\sigma_{1}\right)\right| \leq 4$. We first prove:

Lemma 4.5 Fix a uniform Nash equilibrium $\boldsymbol{\sigma}$. Then, $\left|\operatorname{Supp}\left(\sigma_{1}\right)\right| \neq 4$.

Proof: Assume, by way of contradiction, that $\left|\operatorname{Supp}\left(\sigma_{1}\right)\right|=4$. In this case, strategy 1 is the unique best-response of player 2 to $\sigma_{1}$ since column 1 is the only column containing the maximum number of circles, which is 3 . So, $\operatorname{Supp}\left(\sigma_{2}\right)=\{1\}$. A contradiction to Lemma 4.4. 
We now prove a simple structural property:

Lemma 4.6 (No Column Crossing All Supported Rows Only in Circles) Fix a Nash equilibrium $\boldsymbol{\sigma}$. Then, there is no strategy $s \in \widehat{\Sigma}_{2}$ such that all profiles supported in $\boldsymbol{\sigma}_{-2} \diamond s$ are circles.

Proof: Assume, by way of contradiction, that there is a strategy $s \in \widehat{\Sigma}_{2}$ such that all profiles supported in $\boldsymbol{\sigma}_{-2} \diamond s$ are circles. This implies that $\widehat{\mathrm{U}}_{2}\left(\boldsymbol{\sigma}_{-2} \diamond s\right)=1$. Since $\boldsymbol{\sigma}$ is a Nash equilibrium where player 2 is mixed, Lemma 2.1 (Condition (2)) implies that $\widehat{U}_{2}(\boldsymbol{\sigma}) \geq \widehat{\mathrm{U}}_{2}\left(\boldsymbol{\sigma}_{-2} \diamond s\right)$. Since $\widehat{\mathrm{G}}_{3}$ is win-lose, this implies that $\widehat{\mathrm{U}}_{2}(\boldsymbol{\sigma})=1$. Since $\widehat{\mathrm{G}}_{3}$ is 1 -sum, it follows that $\widehat{\mathrm{U}}_{1}(\boldsymbol{\sigma})=0$. Since $\widehat{\mathrm{G}}_{3}$ has the positive utility property, Lemma 2.2 implies that $\widehat{\mathrm{U}}_{1}(\boldsymbol{\sigma})>0$. A contradiction.

We next prove:

Lemma 4.7 Fix a uniform Nash equilibrium $\boldsymbol{\sigma}$. Then, $\left|\operatorname{Supp}\left(\sigma_{1}\right)\right| \neq 2$.

Proof: Assume, by way of contradiction, that $\left|\operatorname{Supp}\left(\sigma_{1}\right)\right|=2$. Say that two distinct strategies $s, s^{\prime} \in \widehat{\Sigma}_{1}$ for player 1 collide on strategy $t \in \widehat{\Sigma}_{2}$ if both profiles $\langle s, t\rangle$ and $\left\langle s^{\prime}, t\right\rangle$ are circles. Lemma 4.6 implies that the two strategies in $\operatorname{Supp}\left(\sigma_{1}\right)$ do not collide on any strategy in $\widehat{\Sigma}_{2}$. Since column 1 contains only circles except for the entry corresponding to row 4 , Lemma 4.6 implies that $4 \in \operatorname{Supp}\left(\sigma_{1}\right)$. So, three cases need to be considered, corresponding to which the other strategy in $\operatorname{Supp}\left(\sigma_{1}\right)$ is. By inspection, strategies 1 and 4 collide on column 2; strategies 2 and 4 collide on column 4; strategies 3 and 4 collide on column 3. A contradiction.

We finally prove:

Lemma 4.8 Fix a uniform Nash equilibrium $\boldsymbol{\sigma}$. Then, $\left|\operatorname{Supp}\left(\sigma_{1}\right)\right| \neq 3$.

Proof: Assume, by way of contradiction, that $\left|\operatorname{Supp}\left(\sigma_{1}\right)\right|=3$. There are four possibilities corresponding to which strategy $s$ among the four strategies in $\widehat{\Sigma}_{1}$ is excluded from $\operatorname{Supp}\left(\sigma_{1}\right)$. We proceed by case analysis.

1. $s=1$ so that $\operatorname{Supp}\left(\sigma_{1}\right)=\{2,3,4\}$ : Since $\boldsymbol{\sigma}$ is uniform, strategies in $\{1,3,4\}$ are the only three best-responses of player 2 to $\sigma_{1}$ since each of their corresponding columns, restricted to rows corresponding to the strategies in $\operatorname{Supp}\left(\sigma_{1}\right)$, contains the maximum number of circles, which is 2. By Lemma 2.1, it follows that $\operatorname{Supp}\left(\sigma_{2}\right) \subseteq\{1,3,4\}$. Observe that the only square profiles supported in $\boldsymbol{\sigma}$ whenever $\operatorname{Supp}\left(\sigma_{2}\right)=\{1,3,4\}$ are $\langle 4,1\rangle,\langle 2,3\rangle$ and 
$\langle 3,4\rangle$; so, there is exactly one square profile for each possible best-response of player 2 to $\sigma_{1}$. This implies that $\widehat{U}_{1}(\boldsymbol{\sigma})=\frac{1}{3}$, regardless of $\operatorname{Supp}\left(\sigma_{2}\right)$. If $\left|\operatorname{Supp}\left(\sigma_{2}\right)\right|<3$, then for each strategy $s \in \operatorname{Supp}\left(\sigma_{1}\right), \widehat{\mathrm{U}}_{1}\left(\boldsymbol{\sigma}_{-1} \diamond s\right) \geq \frac{1}{2}$, a contradiction to Lemma 2.1 (Condition (1)). So, Supp $\left(\sigma_{2}\right)=\{1,3,4\}$. But then $\widehat{U}_{1}\left(\boldsymbol{\sigma}_{-1} \diamond 1\right)=\frac{2}{3}$, a contradiction to Lemma 2.1 (Condition (1)).

2. $s=2$ so that $\operatorname{Supp}\left(\sigma_{1}\right)=\{1,3,4\}$ : Since $\boldsymbol{\sigma}$ is uniform, strategies in $\{1,2,3\}$ are the only three best-responses for player 2 to $\sigma_{1}$ since each of their corresponding columns, restricted to rows corresponding to the strategies in $\operatorname{Supp}\left(\sigma_{1}\right)$, contains the maximum number of circles, which is 2. By Lemma 2.1, it follows that $\operatorname{Supp}\left(\sigma_{2}\right) \subseteq\{1,2,3\}$. Observe that the only square profiles supported in $\boldsymbol{\sigma}$ whenever $\operatorname{Supp}\left(\sigma_{2}\right)=\{1,2,3\}$ are $\langle 4,1\rangle,\langle 3,2\rangle$; and $\langle 1,3\rangle$; so, there is exactly one square profile for each possible best-response of player 2 to $\sigma_{1}$. This implies that $\widehat{U}_{1}(\boldsymbol{\sigma})=\frac{1}{3}$, regardless of $\operatorname{Supp}\left(\sigma_{2}\right)$. If $\left|\operatorname{Supp}\left(\sigma_{2}\right)\right|<3$, then for each strategy $s \in \operatorname{Supp}\left(\sigma_{1}\right), \widehat{\mathrm{U}}_{1}\left(\boldsymbol{\sigma}_{-1} \diamond s\right) \geq \frac{1}{2}$, a contradiction to Lemma 2.1 (Condition (1)). So, Supp $\left(\sigma_{2}\right)=\{1,2,3\}$. But then $\widehat{U}_{1}\left(\boldsymbol{\sigma}_{-1} \diamond 2\right)=\frac{2}{3}$, a contradiction to Lemma 2.1 (Condition (1)).

3. $s=3$ so that $\operatorname{Supp}\left(\sigma_{1}\right)=\{1,2,4\}$ : Since $\boldsymbol{\sigma}$ is uniform, strategies in $\{1,2,4\}$ are the only three best-responses for player 2 to $\sigma_{1}$ since each of their corresponding columns, restricted to rows corresponding to the strategies in $\operatorname{Supp}\left(\sigma_{1}\right)$, contains the maximum number of circles, which is 2. By Lemma 2.1, it follows that $\operatorname{Supp}\left(\sigma_{2}\right) \subseteq\{1,2,4\}$. Observe that the only square profiles enabled in $\boldsymbol{\sigma}$ whenever $\operatorname{Supp}\left(\sigma_{2}\right)=\{1,2,4\}$ are $\langle 4,1\rangle,\langle 2,2\rangle$ and $\langle 1,4\rangle$; so, there is exactly one square profile for each possible best-response of player 2 to $\sigma_{1}$. This implies that $\widehat{U}_{1}(\boldsymbol{\sigma})=\frac{1}{3}$, regardless of $\operatorname{Supp}\left(\sigma_{2}\right)$. If $\left|\operatorname{Supp}\left(\sigma_{2}\right)\right|<3$, then for each strategy $s \in \operatorname{Supp}\left(\sigma_{1}\right), \widehat{\mathrm{U}}_{1}\left(\boldsymbol{\sigma}_{-1} \diamond s\right) \geq \frac{1}{2}$, a contradiction to Lemma 2.1 (Condition (1)). So, Supp $\left(\sigma_{2}\right)=\{1,2,4\}$. But then $\widehat{U}_{1}\left(\boldsymbol{\sigma}_{-1} \diamond 3\right)=\frac{2}{3}$, a contradiction to Lemma 2.1 (Condition (1)).

4. $s=4$ so that $\operatorname{Supp}\left(\sigma_{1}\right)=\{1,2,3\}$ : Then, all profiles supported in $\boldsymbol{\sigma}_{-2} \diamond 1$ are circles. A contradiction to Lemma 4.6.

The proof is now complete.

The claim follows now from Lemmas 4.4, 4.5, 4.7 and 4.8,

By Proposition 4.3, the win-lose game $\widehat{G}_{3}$ is a negative instance for $\exists$ UNIFORM NASH. 


\subsection{The Non-Symmetric Game $\widehat{G}_{4}$}

Define the win-lose bimatrix game $\widehat{\mathrm{G}}_{4}=\left\langle[2],\left\{\widehat{\Sigma}_{i}\right\}_{i \in[2]},\left\{\widehat{\mathrm{U}}_{i}\right\}_{i \in[2]}\right\rangle$ with $\widehat{\Sigma}_{1}=\widehat{\Sigma}_{2}=\{1,2,3\}$; the utility functions are depicted below:

\begin{tabular}{||c|c||c|c||c|c||}
\hline Profile $\mathbf{s}$ & $\widehat{\mathrm{U}}(\mathbf{s})$ & Profile $\mathbf{s}$ & $\widehat{\mathrm{U}}(\mathbf{s})$ & Profile $\mathbf{s}$ & $\widehat{\mathrm{U}}(\mathbf{s})$ \\
\hline \hline$\langle 1,1\rangle$ & $\langle 1,0\rangle$ & $\langle 1,2\rangle$ & $\langle 0,1\rangle$ & $\langle 1,3\rangle$ & $\langle 1,0\rangle$ \\
\hline$\langle 2,1\rangle$ & $\langle 0,1\rangle$ & $\langle 2,2\rangle$ & $\langle 0,0\rangle$ & $\langle 2,3\rangle$ & $\langle 1,0\rangle$ \\
\hline$\langle 3,1\rangle$ & $\langle 0,1\rangle$ & $\langle 3,2\rangle$ & $\langle 1,0\rangle$ & $\langle 3,3\rangle$ & $\langle 0,1\rangle$ \\
\hline \hline
\end{tabular}

Clearly, $\widehat{G}_{4}$ has the positive utility property. We prove:

Proposition $4.9 \widehat{\mathrm{G}}_{4}$ has no symmetric Nash equilibrium.

Proof: Assume, by way of contradiction, that $\widehat{\mathrm{G}}_{4}$ has a symmetric Nash equilibrium $\boldsymbol{\sigma}$. Clearly, $\widehat{G}_{4}$ has no pure Nash equilibrium. Hence, four cases need to be examined:

(1) For each player $i \in[2], \operatorname{Supp}\left(\sigma_{i}\right)=\{1,2\}$ : Then, $\widehat{\mathrm{U}}_{1}\left(\boldsymbol{\sigma}_{-1} \diamond 2\right)=0$. Since $2 \in \operatorname{Supp}\left(\sigma_{1}\right)$, Lemma 2.1 (Condition (1)) implies that $\widehat{U}_{1}(\boldsymbol{\sigma})=0$. A contradiction to Lemma 2.2.

(2) For each player $i \in[2], \operatorname{Supp}\left(\sigma_{i}\right)=\{2,3\}$ : Then, $\widehat{U}_{2}\left(\boldsymbol{\sigma}_{-2} \diamond 2\right)=0$. Since $2 \in \operatorname{Supp}\left(\sigma_{2}\right)$, Lemma 2.1 (Condition (1)) implies that $\widehat{U}_{2}(\boldsymbol{\sigma})=0$. A contradiction to Lemma 2.2.

(3) For each player $i \in[2], \operatorname{Supp}\left(\sigma_{i}\right)=\{1,3\}$ : Then, $\widehat{U}_{1}\left(\sigma_{-1} \diamond 3\right)=0$. Since $3 \in \operatorname{Supp}\left(\sigma_{1}\right)$, Lemma 2.1 (Condition (1)) implies that $\widehat{U}_{1}(\boldsymbol{\sigma})=0$. A contradiction to Lemma 2.2.

(4) For each player $i \in[2], \operatorname{Supp}\left(\sigma_{i}\right)=\{1,2,3\}$ : Then, $\widehat{U}_{1}\left(\boldsymbol{\sigma}_{-1} \diamond 1\right)=\sigma_{2}(1)+\sigma_{2}(3)$ and $\widehat{U}_{1}\left(\boldsymbol{\sigma}_{-1} \diamond\right.$ $2)=\sigma_{2}(3)$. Since $\sigma_{2}(1)>0$, it follows that $\widehat{\mathrm{U}}_{1}\left(\boldsymbol{\sigma}_{-1} \diamond 1\right)>\widehat{\mathrm{U}}_{1}\left(\boldsymbol{\sigma}_{-1} \diamond 2\right)$. Since $1,2 \in$ $\operatorname{Supp}\left(\sigma_{1}\right)$, Lemma 2.1 (Condition (1)) implies that $\widehat{U}_{1}\left(\boldsymbol{\sigma}_{-1} \diamond 1\right)=\widehat{U}_{1}\left(\boldsymbol{\sigma}_{-1} \diamond 2\right)$. A contradiction.

The proof is complete.

By Proposition 4.9, the win-lose game $\widehat{\mathrm{G}}_{4}$ is a negative instance for $\exists$ SYMMETRIC NASH.

\subsection{The Diagonal Game $\widehat{G}_{5}[k]$}

Fix an integer $k \geq 1$. Denote as $\mathrm{D}_{k}$ the $k \times k$ win-lose matrix such that $\mathrm{D}_{k}[i, j]=1$ if and only if $i \leq j$, with $1 \leq i, j \leq k$; so, $\mathrm{D}_{k}$ is an upper-diagonal matrix. Then, $\mathrm{D}_{k}^{\mathrm{T}}$ is a $k \times k$ win-lose matrix such that $\mathrm{R}_{k}^{\mathrm{T}}[i, j]=1$ if and only if $j \leq i$, with $1 \leq i, j \leq k$; so, $\mathrm{D}_{k}^{\mathrm{T}}$ is a lowerdiagonal matrix. Define the win-lose bimatrix game $\widehat{G}_{5}[k]:=\left\langle D_{k}, D_{k}^{T}\right\rangle$, which is symmetric by construction. Clearly, $\widehat{G}_{5}[k]$ has the positive utility property. We prove: 
Proposition 4.10 Fix an integer $k \geq 1$. Then, $\widehat{\mathrm{G}}_{5}[k]$ has exactly $k$ Nash equilibria, which are Pareto-Optimal, Strongly Pareto-Optimal and symmetric, each yielding utility 1 to each player.

Proof: Note that $\widehat{\mathrm{G}}_{5}[k]$ has exactly $k$ pure Nash equilibria $\mathbf{s}^{s}$, with $s \in[k]$, where each player $i \in[2]$ chooses strategy $s$ in $\mathbf{s}^{s}$ to get utility 1 . Note that each of the $k$ pure equilibria is ParetoOptimal, Strongly Pareto-Optimal and symmetric. Hence, it remains to prove that $\widehat{G}_{5}[k]$ has no mixed Nash equilibrium. Towards this end, assume, by way of contradiction, that $\widehat{\mathrm{G}}_{5}[k]$ has a mixed Nash equilibrium $\boldsymbol{\sigma}$. For each player $i \in[2]$, set $s_{i}^{*}:=\min \operatorname{Supp}\left(\sigma_{i}\right)$; so, $s_{1}^{*}$ (resp., $s_{2}^{*}$ ) is the row (resp., column) with least index played by the row (resp., column) player in $\boldsymbol{\sigma}$. By the definitions of $\mathrm{D}_{k}$ and $\mathrm{D}_{k}^{\mathrm{T}}$, it follows that the set of best-response strategies for the row (resp., column) player is $\left[s_{2}^{*}\right]$ (resp., $\left[s_{1}^{*}\right]$ ); thus, by Lemma 2.1, $\operatorname{Supp}\left(\sigma_{1}\right) \subseteq\left[s_{2}^{*}\right]$ and $\operatorname{Supp}\left(\sigma_{2}\right) \subseteq\left[s_{1}^{*}\right]$. These imply together that $s_{1}^{*} \leq s_{2}^{*}$ and $s_{2}^{*} \leq s_{1}^{*}$, so that $s_{1}^{*}=s_{2}^{*}$. Hence, for each player $i \in[2]$, $\operatorname{Supp}\left(\sigma_{i}\right) \subseteq\left[s_{i}^{*}\right]=\left[\min \operatorname{Supp}\left(\sigma_{i}\right)\right]$, which implies that $\operatorname{Supp}\left(\sigma_{i}\right)=\left\{s_{i}^{*}\right\}$. Hence, $\boldsymbol{\sigma}$ is a pure Nash equilibrium. A contradiction.

By Proposition 4.10, the win-lose game $\widehat{G}_{5}[k]$ is a negative instance for $\exists k+1 \mathrm{NASH}$, with $k \geq 1$, for $\exists \neg$ PARETO-OPTIMAL NASH, for $\exists \neg$ STRONGLY PARETO-OPTIMAL NASH and for $\exists \neg$ SYMMETRIC NASH.

\section{The Win-Lose Reduction}

We start with some preliminary material. Denote $I_{n}:=\{0,1, \ldots, n-1\}$; for all arithmetic operations on $I_{n}$, we shall assume a cyclic ordering on its elements; so, $(n-1)+1=0$ and for a given pair $i, j \in I_{n}, j-i$ is the number of elements of $I_{n}$ encountered when moving from $j$ (excluded) to $i$ (included) in the anticlockwise direction. A CNF SAT formula is a boolean formula $\phi=\bigwedge_{i \in[k]} \bigvee_{j \in\left[l_{i}\right]} \ell_{i j}$ in Conjunctive Normal Form, where $\ell_{i j}$ is a literal (a boolean variable or its negation), we shall denote as $(i) \mathcal{C}=\mathcal{C}(\phi)=\left\{\bigvee_{j \in\left[l_{i}\right]} \ell_{i j} \mid i \in[k]\right\}$, the set of clauses, and (ii) $\operatorname{Var}=\operatorname{Var}(\phi)=\left\{v_{0}, \ldots, v_{n-1}\right\}$ and $\mathrm{L}=\mathrm{L}(\phi)=\left\{\ell_{0}, \bar{\ell}_{0}, \ldots, \ell_{n-1}, \bar{\ell}_{n-1}\right\}$ the sets of variables and literals, respectively, with $|\operatorname{Var}(\phi)|=n$ and $|\mathrm{L}(\phi)|=2 n$. We shall use c to denote a clause from $\mathcal{C}(\phi)$, and either $\ell$ or $\bar{\ell}$ to denote a literal from $\mathrm{L}(\phi) . \phi$ is a 3SAT formula if each clause $\mathrm{c}$ has $|\mathrm{c}|=3$. An assignment is a function $\gamma: \operatorname{Var}(\phi) \rightarrow\{0,1\}$; so, $\gamma$ is represented by the $n$-tuple of literals made true. For a literal $\ell \in \mathrm{L}(\phi)$, denote as $I(\ell)$ the index $j \in I_{n}$ such that $\ell \in\left\{\ell_{j}, \bar{\ell}_{j}\right\}$. Denote as $\phi(\gamma)$ the value of $\phi$ when each variable $v$ takes the value $\gamma(v)$. $\gamma$ is a satisfying assignment if $\phi(\gamma)=1$, and $\phi$ is satisfiable if it has a satisfying assignment; deciding the satisfiability of a 3SAT formula $\phi$ is $\mathcal{N} \mathcal{P}$-complete. Denote as $\# \phi$ and $\oplus \phi$ the number and the parity of the number of satisfying assignments of $\phi$, respectively; when $\phi$ is a 3SAT formula, computing $\# \phi$ is $\# \mathcal{P}$-complete [35] and computing $\oplus \phi$ is $\oplus \mathcal{P}$-complete 33 . 


\subsection{The Reduction}

We shall construct, given an $r$-player win-lose gadget game $\widehat{\mathrm{G}}$, for an arbitrary integer $r \geq 2$, and an instance $\phi$ of 3SAT with $n=|\operatorname{Var}(\phi)| \geq 4$, the $r$-player win-lose game $\mathrm{G}=\mathrm{G}(\widehat{\mathrm{G}}, \phi)=$ $\left\langle[r],\left\{\Sigma_{i}\right\}_{i \in[r]},\left\{\bigcup_{i}\right\}_{i \in[r]}\right\rangle$. Players 1 and 2 are called special. For a player $i \in[2], \bar{i} \in[2] \backslash\{i\}$ denotes the special player other than $i$.

\subsubsection{Inadequacy of the Reduction in [13] and Getting Around it}

A crucial step in both the win-lose reduction and the reduction in [13] is to guarantee two significant properties:

(P.1) A mixed profile in which some special player chooses some literals with positive probability is a Nash equilibrium for the constructed game $\mathrm{G}$ if and only if $\phi$ is satisfiable.

(P.2) Each satisfying assignment induces a Nash equilibrium where both special players are choosing the literals from the satisfying assignment with uniform probabilities.

To guarantee these properties, both the win-lose reduction and the reduction in [13] have to rule out the existence of a Nash equilibrium in which either some special player plays literals with non-uniform probabilities or some pair of complementary literals $(\ell$ and $\bar{\ell})$ is not played by some special player. The reduction in [13] achieves these properties with the following approach:

- By assigning a non-zero utility to both special players when they are choosing a pair of non-complementary literals.

- By using $n$ auxiliary strategies, called variables, $\mathrm{v}_{0}, \ldots, \mathrm{v}_{n-1}$, where, for each index $j \in I_{n}$, $\mathrm{v}_{j}$ is associated with the pair of complementary literals $\left\{\ell_{j}, \bar{\ell}_{j}\right\}$, as follows: When a special player $i \in[2]$ chooses a literal $\ell \in\left\{\ell_{j}, \bar{\ell}_{j}\right\}$, the utility given to player $\bar{i}$ when choosing a variable other than $v_{j}$ is more than the utility she would get when choosing any literal from $\mathrm{L}(\phi)$.

Thus, this approach requires using at least two different non-zero utilities. Hence, the reduction in [13] is inadequate for win-lose games. We resolve this inadequacy by combining two new ideas in the win-lose reduction:

- Instead of giving non-zero utility to both special players when they are choosing noncomplementary literals, only one of them gets non-zero utility. This idea is implemented by using a cyclic ordering among the literals and extending the construction of the cyclic 
game $\widehat{G}_{1}[n]$. (See Cases (1) through (4) in Figure 2.) We shall prove that this suffices to rule out the existence of Nash equilibria in which literals in the support of both special players are played with non-uniform probabilities.

- To guarantee that every pair of complementary literals are chosen with strictly positive probability, we introduce a new kind of auxiliary strategies, namely the pair variables $\mathrm{v}_{j, k}$, for each pair $j, k \in I_{n}$ with $j \neq k$; thus, there are $n(n-1)$ of them. The pair variables provide the special player $\bar{i}$ with an improving deviation whenever there is a pair of complementary literals not chosen by the special player $i$; at the same time, we define the utility functions so that no special player could improve by switching to a pair variable when both special players are playing the same satisfying assignment with uniform probabilities. (See Cases $(5 / a)$ and $(5 / b)$ in Figure 2 ) This is the point where the structure of a 3SAT formula shall be needed.

The proof of Lemma 5.5 demonstrates how the two new ideas combine together. We now proceed to formally define the win-lose reduction.

\subsubsection{Formal Definition}

For each player $i \in[2], \Sigma_{i}:=\widehat{\Sigma}_{i} \cup \mathrm{L}(\phi) \cup \mathcal{C}(\phi) \cup \mathrm{V}(\phi)$; for each player $i \in[r] \backslash[2], \Sigma_{i}:=\widehat{\Sigma}_{i} \cup\{\delta\}$, where $\mathrm{V}(\phi)=\left\{\mathrm{v}_{i, j} \mid 0 \leq i, j<n, i \neq j\right\}$ and $\delta$ is a special strategy. The strategies in $\mathrm{V}(\phi)$ are called pair variables. The two special players 1 and 2 are the only players whose sets of strategies are determined by the formula $\phi$. Note that for each player $i \in[2],\left|\Sigma_{i}\right|=$ $\left|\widehat{\Sigma}_{i}\right|+2 n+|\mathcal{C}(\phi)|+n(n+1)$, and for each player $i \in[r] \backslash[2],\left|\Sigma_{i}\right|=\left|\widehat{\Sigma}_{i}\right|+1$; so, the size of the strategy sets in $\mathrm{G}(\widehat{\mathrm{G}}, \phi)$ is polynomial in the sizes of $\widehat{\mathrm{G}}$ and $\phi$. In the following, we shall write $\mathrm{L}, \mathcal{C}$ and $\mathrm{V}$ for $\mathrm{L}(\phi), \mathcal{C}(\phi)$, and $\mathrm{V}(\phi)$, respectively.

Fix a profile $\mathbf{s}=\left\langle s_{1}, \ldots, s_{r}\right\rangle$ from $\Sigma=\Sigma_{1} \times \ldots \times \Sigma_{r}$. Use $\mathbf{s}$ to partition $[r]$ into $\widehat{\mathcal{P}}(\mathbf{s})=\{i \in[r] \mid$ $\left.s_{i} \in \widehat{\Sigma}_{i}\right\}$ and $\mathcal{P}(\mathbf{s})=\left\{i \in[r] \mid s_{i} \notin \widehat{\Sigma}_{i}\right\}$; so, $\widehat{\mathcal{P}}(\mathbf{s})$ and $\mathcal{P}(\mathbf{s})$ are the sets of players choosing and not choosing, respectively, strategies from $\widehat{G}$. The utility functions are depicted in Figure 2 .

\subsubsection{Notation}

For a mixed profile $\boldsymbol{\sigma}$ and a special player $i \in[2], \sigma_{i}(S)$ denotes the total probability assigned by $\sigma_{i}$ to strategies from $S \subseteq \Sigma_{i}$; so, in particular, $\sigma_{i}(\mathrm{~L}), \sigma_{i}(\mathcal{C} \cup \mathrm{V})$ and $\sigma_{i}\left(\widehat{\Sigma}_{i}\right)$ are the total probabilities assigned by $\sigma_{i}$ to strategies from $\mathrm{L}, \mathcal{C} \cup \mathrm{V}$ and $\widehat{\Sigma}_{i}$, respectively, with $\sigma_{i}(\mathrm{~L})+\sigma_{i}(\mathcal{C} \cup$ $\mathrm{V})+\sigma_{i}\left(\widehat{\Sigma}_{i}\right)=1$. For a player $i \in[r] \backslash[2], \sigma_{i}\left(\widehat{\Sigma}_{i}\right)$ and $\sigma_{i}(\delta)$, with $\sigma_{i}\left(\widehat{\Sigma}_{i}\right)+\sigma_{i}(\delta)=1$, are set correspondingly. Sometimes a strategy from $\widehat{G}$ will be called a gadget strategy. 


\begin{tabular}{||l|l|l||}
\hline \hline Case & Condition on the profile $\mathbf{s}=\left\langle s_{1}, s_{2}, \ldots, s_{r}\right\rangle$ & Utility vector $\mathrm{U}(\mathbf{s})$ \\
\hline \hline$(1)$ & $\mathbf{s}=\langle\ell, \bar{\ell}, \delta, \ldots, \delta\rangle$ & $\langle 0,0,1, \ldots, 1\rangle$ \\
\hline$(2)$ & $\mathbf{s}=\left\langle\ell, \ell^{\prime}, \delta, \ldots, \delta\right\rangle$ with $I\left(\ell^{\prime}\right)-I(\ell) \in\{0,1\}$ and $\ell \neq \bar{\ell}$ & $\langle 1,0,1, \ldots, 1\rangle$ \\
\hline$(3)$ & $\mathbf{s}=\left\langle\ell, \ell^{\prime}, \delta, \ldots, \delta\right\rangle$ with $I\left(\ell^{\prime}\right)-I(\ell) \in\{2,3\}$ & $\langle 0,1,1, \ldots, 1\rangle$ \\
\hline$(4)$ & $\mathbf{s}=\left\langle\ell, \ell^{\prime}, \delta, \ldots, \delta\right\rangle$ with $I\left(\ell^{\prime}\right)-I(\ell)>3$ & $\langle 0,0,1, \ldots, 1\rangle$ \\
\hline \hline$(5 / \mathrm{a})$ & $\mathbf{s}=\left\langle\mathrm{v}_{i j}, \ell_{k}, \delta, \ldots, \delta\right\rangle$ with $k \in\{i, j\}$ & $\langle 1,0,1, \ldots, 1\rangle$ \\
\hline \hline$(5 / \mathrm{b})$ & $\mathbf{s}=\left\langle\ell_{k}, \mathrm{v}_{i j}, \delta, \ldots, \delta\right\rangle$ with $k \in\{i, j\}$ & $\langle 0,1,1, \ldots, 1\rangle$ \\
\hline \hline$(6 / \mathrm{a})$ & $\mathbf{s}=\langle\mathrm{c}, \ell, \delta, \ldots, \delta\rangle$ with $\bar{\ell} \in \mathrm{c}$ & $\langle 1,0,1, \ldots, 1\rangle$ \\
\hline \hline$(6 / \mathrm{b})$ & $\mathbf{s}=\langle\ell, \mathrm{c}, \delta, \ldots, \delta\rangle$ with $\bar{\ell} \in \mathrm{c}$ & $\langle 0,1,1, \ldots, 1\rangle$ \\
\hline \hline$(7)$ & For each $i \in[r], s_{i} \in \widehat{\Sigma}_{i} .(\mathrm{So},|\widehat{\mathcal{P}}(\mathbf{s})|=r)$. & $\widehat{\mathrm{U}}\left(\left\langle s_{1}, \ldots, s_{r}\right\rangle\right)$ \\
\hline \hline$(8)$ & $|\widehat{\mathcal{P}}(\mathbf{s})|>1$ and $|\mathcal{P}(\mathbf{s})|>0$ & $\mathrm{U}_{i}(\mathbf{s})=1$ iff $i \in \widehat{\mathcal{P}}(\mathbf{s})$ \\
\hline$(9)$ & $\widehat{\mathcal{P}}(\mathbf{s})=\{i\}$ with $i \in[r] \backslash[2]$ & $\mathrm{U}_{j}(\mathbf{s})=\delta_{j i}$ \\
\hline$(10)$ & $\widehat{\mathcal{P}}(\mathbf{s})=\{i\}$ with $i \in[2] \& s_{[2] \backslash\{i\}} \in\left\{\ell_{0}, \bar{\ell}_{0}, \ell_{1}, \bar{\ell}_{1}\right\} \cup \mathcal{C} \cup \mathrm{V}$ & $\mathrm{U}_{j}(\mathbf{s})=\delta_{j i}$ \\
\hline \hline$(11)$ & None of the above. & $\langle 0, \ldots, 0\rangle$ \\
\hline \hline
\end{tabular}

Figure 2: The utility functions for the game $\mathrm{G}=\mathrm{G}(\widehat{\mathrm{G}}, \phi) .\left(\delta_{i j}\right.$ denotes the Kronecker delta.)

\subsection{Properties of Nash Equilibria for G}

We now present a collection of properties for a Nash equilibrium $\sigma \in \mathcal{N} \mathcal{E}(\mathrm{G})$. The first property is that either all players are exclusively playing gadget strategies or none is.

Lemma 5.1 Fix a Nash equilibrium $\boldsymbol{\sigma} \in \mathcal{N} \mathcal{E}(\mathrm{G})$ with $\operatorname{Supp}\left(\sigma_{i}\right) \subseteq \widehat{\Sigma}_{i}$ for some player $i \in[r]$. Then, for each player $j \in[r] \backslash\{i\}$, Supp $\left(\sigma_{j}\right) \subseteq \widehat{\Sigma}_{j}$.

Proof: Fix a player $j \in[r] \backslash\{i\}$ and a strategy $s \in \operatorname{Supp}\left(\sigma_{j}\right)$. Since $\boldsymbol{\sigma}$ is a Nash equilibrium, $s$ is a best-response to $\boldsymbol{\sigma}_{-j}$. We shall establish that $s \in \widehat{\Sigma}_{j}$. Consider the mixed profile $\boldsymbol{\sigma}_{-j} \diamond s$. Since Supp $\left(\sigma_{i}\right) \subseteq \widehat{\Sigma}_{i}$, each profile $\mathbf{s}_{-j} \diamond s$ supported in $\boldsymbol{\sigma}_{-j} \diamond s$ may only come from Cases (7), (8), (9), (10) and (11). For any such profile $\mathbf{s}_{-j} \diamond s$, it follows, by the utility functions, that if $s \notin \widehat{\Sigma}_{j}$, then $\mathrm{U}_{j}\left(\mathbf{s}_{-j} \diamond s\right)=0$, which implies that $\mathrm{U}_{j}\left(\boldsymbol{\sigma}_{-j} \diamond s\right)=0$. So, to establish the claim, it suffices to determine a strategy $s \in \widehat{\Sigma}_{j}$ with $\bigcup_{j}\left(\boldsymbol{\sigma}_{-j} \diamond s\right)>0$. We proceed by case analysis.

- Assume first that there is a profile $\mathbf{s}_{-j}$ supported in $\boldsymbol{\sigma}_{-j}$ with $\mathbf{s}_{-j} \in \widehat{\Sigma}_{-j}$; hence, by the positive utility property of $\widehat{\mathrm{G}}$, there is a strategy $t=t\left(\mathbf{s}_{-j}\right) \in \widehat{\Sigma}_{j}$ with $\widehat{\mathrm{U}}_{j}\left(\mathbf{s}_{-j} \diamond t\right)>0$. 
Set $s:=t$. Then,

$$
\begin{aligned}
& \mathrm{U}_{j}\left(\boldsymbol{\sigma}_{-j} \diamond s\right) \\
& \geq \mathbb{P}_{\mathbf{s}_{-j} \sim \sigma_{-j}}\left(\mathbf{s}_{-j} \diamond s\right) \cdot \mathbf{U}_{j}\left(\mathbf{s}_{-j} \diamond s\right) \quad \text { (since } \mathrm{G} \text { is win-lose) } \\
& =\underbrace{\mathbb{P}_{\mathbf{s}_{-j} \sim \boldsymbol{\sigma}_{-j}}\left(\mathbf{s}_{-j} \diamond s\right)}_{>0} \cdot \underbrace{\widehat{U}_{j}\left(\mathbf{s}_{-j} \diamond s\right)}_{>0} \quad \text { (by the utility functions) } \\
& >0 \text {. }
\end{aligned}
$$

- Assume now that there is no profile $\mathbf{s}_{-j}$ supported in $\boldsymbol{\sigma}_{-j}$ with $\mathbf{s}_{-j} \in \widehat{\Sigma}_{-j}$. Set $s$ to any strategy from $\widehat{\Sigma}_{j}$. Thus, $\{i, j\} \subseteq \widehat{\mathcal{P}}\left(\mathbf{s}_{-j} \diamond s\right)$ for each profile $\mathbf{s}_{-j} \diamond s$ supported in $\boldsymbol{\sigma}_{-j} \diamond s$; so, by the utility functions, only profiles from Case (8) may be supported in $\sigma_{-j} \diamond s$. Since $s \in \widehat{\Sigma}_{j}, j \in \widehat{\mathcal{P}}\left(\mathbf{s}_{-j} \diamond s\right)$. Hence, by the utility functions, $\mathbf{U}_{j}\left(\mathbf{s}_{-j} \diamond s\right)=1$, which implies that $\mathrm{U}_{j}\left(\boldsymbol{\sigma}_{-j} \diamond s\right)=1>0$.

The claim follows.

The next property concerns the two special players: if one of them is assigning probability 0 on literals, then the other is exclusively playing gadget strategies.

Lemma 5.2 Fix a Nash equilibrium $\boldsymbol{\sigma} \in \mathcal{N} \mathcal{E}(\mathrm{G})$ with $\operatorname{Supp}\left(\sigma_{i}\right) \subseteq \widehat{\Sigma}_{i} \cup \mathcal{C} \cup \vee$ for some special player $i \in[2]$. Then, $\operatorname{Supp}\left(\sigma_{\bar{i}}\right) \subseteq \widehat{\Sigma}_{\bar{i}}$.

Proof: Fix a strategy $s \in \operatorname{Supp}\left(\sigma_{\bar{i}}\right)$. Since $\boldsymbol{\sigma}$ is a Nash equilibrium, $s$ is a best-response to $\boldsymbol{\sigma}_{-\bar{i}}$. We shall establish that $s \in \widehat{\Sigma}_{\bar{i}}$. Consider the mixed profile $\boldsymbol{\sigma}_{-\bar{i}} \diamond s$. Since $\operatorname{Supp}\left(\sigma_{i}\right) \subseteq \widehat{\Sigma}_{i} \cup \mathcal{C} \cup \mathrm{V}$, or $\operatorname{Supp}\left(\sigma_{i}\right) \cap \mathbf{L}=\emptyset$, each profile $\mathbf{s}_{-\bar{i}} \diamond s$ supported in $\boldsymbol{\sigma}_{-\bar{i}} \diamond s$ may only come from Cases (5), (6), (7), (8), (9), (10) and (11). For any such profile $\mathbf{s}_{-\bar{i}} \diamond s$, it follows, by the utility functions, that if $s \notin \widehat{\Sigma}_{\bar{i}}$, then $\mathbf{U}_{\bar{i}}\left(\mathbf{s}_{-\bar{i}} \diamond s\right)=0$, which implies that $\mathrm{U}_{\bar{i}}\left(\boldsymbol{\sigma}_{-\bar{i}} \diamond s\right)=0$. (Note that Cases (5) and (6) occur if and only if player $\bar{i}$ chooses a strategy from L.) So, to establish the claim, it suffices to determine a strategy $s \in \widehat{\Sigma}_{\bar{i}}$ with $\bigcup_{\bar{i}}\left(\boldsymbol{\sigma}_{-\bar{i}} \diamond s\right)>0$. We proceed by case analysis.

- Assume that there is a profile $\mathbf{s}$ supported in $\boldsymbol{\sigma}$ with $\mathbf{s}_{-\bar{i}} \in \widehat{\Sigma}_{-\bar{i}}$. By the positive utility property of $\widehat{\mathrm{G}}$, there is a strategy $t=t\left(\mathbf{s}_{-\bar{i}}\right) \in \widehat{\Sigma}_{\bar{i}}$ with $\mathrm{U}_{\bar{i}}\left(\mathbf{s}_{-\bar{i}} \diamond t\right)>0$. Set $s:=t$. Then,

$$
\begin{aligned}
& \mathrm{U}_{\bar{i}}\left(\sigma_{-\bar{i}} \diamond s\right) \\
& \geq \mathbb{P}_{\mathbf{s}_{-\bar{i}} \sim \sigma_{-\bar{i}}}\left(\mathbf{s}_{-\bar{i}} \diamond s\right) \cdot \mathrm{U}_{\bar{i}}\left(\mathbf{s}_{-\bar{i}} \diamond s\right) \quad \text { (since } \mathrm{G} \text { is win-lose) } \\
& =\underbrace{\mathbb{P}_{\mathbf{s}_{-\bar{i}} \sim \sigma_{-\bar{i}}}\left(\mathbf{s}_{-\bar{i}} \diamond s\right)}_{>0} \cdot \underbrace{\widehat{U}_{\bar{i}}\left(\mathbf{s}_{-\bar{i}} \diamond s\right)}_{>0} \text { (by the utility functions) } \\
& >\quad 0 \text {. }
\end{aligned}
$$


- Assume now that there is no profile s supported in $\boldsymbol{\sigma}$ with $\mathbf{s}_{-\bar{i}} \in \widehat{\Sigma}_{-\bar{i}}$. Define $s$ to be any strategy from $\widehat{\Sigma}_{\bar{i}}$. So, $\bar{i} \in \widehat{\mathcal{P}}\left(\mathbf{s}_{-\bar{i}} \diamond s\right)$ for each profile $\mathbf{s}_{-\bar{i}}$ supported in $\boldsymbol{\sigma}_{-\bar{i}} \diamond s$; it follows that only profiles from Cases (8) and (10) may be supported in $\sigma_{-\bar{i}} \diamond s$. Hence, by the utility functions, $\mathbf{U}_{\bar{i}}\left(\mathbf{s}_{-\bar{i}} \diamond s\right)=1$, which implies that $\mathbf{U}_{\bar{i}}\left(\boldsymbol{\sigma}_{-\bar{i}} \diamond s\right)=1>0$.

The claim follows.

We now prove that the only Nash equilibria for $G$ where some player is exclusively playing gadget strategies are the Nash equilibria for the gadget game $\widehat{G}$.

Lemma 5.3 The following conditions hold:

(C.1) $\mathcal{N} \mathcal{E}(\widehat{G}) \subseteq \mathcal{N} \mathcal{E}(\mathrm{G})$.

(C.2) There is no Nash equilibrium $\boldsymbol{\sigma} \in \mathcal{N} \mathcal{E}(\mathrm{G}) \backslash \mathcal{N} \mathcal{E}(\widehat{\mathrm{G}})$ with $\operatorname{Supp}\left(\sigma_{i}\right) \subseteq \widehat{\Sigma}_{i}$, for some player $i \in[r]$.

Proof: For Condition (C.1), fix a Nash equilibrium $\sigma \in \mathcal{N} \mathcal{E}(\widehat{\mathrm{G}})$; so, no player $i \in[r]$ can improve by switching to a strategy $s \in \widehat{\Sigma}_{i}$. Consider now player $i \in[r]$ switching to a strategy $s \notin \widehat{\Sigma}_{i}$. Then, a profile s supported in $\boldsymbol{\sigma}_{-i} \diamond s$ may only come from Cases (8), (9), (10) and (11), so that $i \in \mathcal{P}(\mathbf{s})$ and $\mathbf{U}_{i}(\mathbf{s})=0$, which implies that $\mathbf{U}_{i}\left(\boldsymbol{\sigma}_{-i} \diamond s\right)=0$. Since $\mathrm{G}$ is win-lose, $\mathrm{U}_{i}(\boldsymbol{\sigma}) \geq 0$. It follows that $\bigcup_{i}\left(\boldsymbol{\sigma}_{-i} \diamond s\right) \leq \mathbf{U}_{i}(\boldsymbol{\sigma})$, and player $i$ cannot improve by switching to $s$. So, $\sigma$ is a Nash equilibrium for $\mathrm{G}$, so that $\mathcal{N} \mathcal{E}(\widehat{\mathrm{G}}) \subseteq \mathcal{N} \mathcal{E}(\mathrm{G})$.

For Condition (C.2), assume, by way of contradiction, that there is a Nash equilibrium $\boldsymbol{\sigma} \in \mathcal{N} \mathcal{E}(\mathrm{G}) \backslash \mathcal{N} \mathcal{E}(\widehat{\mathrm{G}})$ with $\operatorname{Supp}\left(\sigma_{i}\right) \subseteq \widehat{\Sigma}_{i}$ for some player $i \in[r]$. By Lemma 5.1, for each player $j \in[r] \backslash\{i\}$, Supp $\left(\sigma_{j}\right) \subseteq \widehat{\Sigma}_{j}$. Hence, $\boldsymbol{\sigma}$ is a mixed profile for $\widehat{\mathrm{G}}$, which implies that $\boldsymbol{\sigma} \in \mathcal{N} \mathcal{E}(\widehat{\mathrm{G}})$. A contradiction.

A Nash equilibrium for $G$ coming from $\widehat{G}$ will be called a gadget equilibrium. The following properties characterize a Nash equilibrium $\sigma \in \mathcal{N} \mathcal{E}(\mathrm{G}) \backslash \mathcal{N} \mathcal{E}(\widehat{\mathrm{G}})$. Lemmas [5.4, 5.5, 5.10] and 5.11] concern the two special players; Lemma 5.9] concerns the non-special players. We first prove that each special player is playing some literal with positive probability.

Lemma 5.4 Fix a Nash equilibrium $\boldsymbol{\sigma} \in \mathcal{N} \mathcal{E}(\mathrm{G}) \backslash \mathcal{N} \mathcal{E}(\widehat{\mathrm{G}})$. Then, $\sigma_{1}(\mathrm{~L}) \cdot \sigma_{2}(\mathrm{~L})>0$.

Proof: Assume, by way of contradiction, that there is a player $i \in[2]$ with $\sigma_{i}(\mathrm{~L})=0$. So, $\operatorname{Supp}\left(\sigma_{i}\right) \subseteq \widehat{\Sigma}_{i} \cup \mathcal{C} \cup \mathrm{V}$. Then, Lemma 5.2 implies that $\operatorname{Supp}\left(\sigma_{\bar{i}}\right) \subseteq \widehat{\Sigma}_{\bar{i}}$. A contradiction to Lemma 5.3 (Condition (2)). 
We now prove that in a Nash equilibrium $\sigma \in \mathcal{N E}(\mathrm{G}) \backslash \mathcal{N} \mathcal{E}(\widehat{\mathrm{G}})$, each special player assigns the same positive probability to each pair of literals $\{\ell, \bar{\ell}\}$.

Lemma 5.5 Fix a Nash equilibrium $\boldsymbol{\sigma} \in \mathcal{N} \mathcal{E}(\mathrm{G}) \backslash \mathcal{N E}(\widehat{\mathrm{G}})$ and a special player $i \in$ [2]. Then, for each literal $\ell \in \mathrm{L}, \sigma_{i}(\{\ell, \bar{\ell}\})=\frac{\sigma_{i}(\mathrm{~L})}{n}$.

Proof: We shall establish a sequence of claims. We first prove:

Claim 5.6 For each special player $i \in[2]$, there is no index $j \in I_{n}$ with $\sigma_{i}(\mathrm{~L})=\sigma_{i}\left(\left\{\ell_{j}, \bar{\ell}_{j}\right\}\right)$.

Proof: Assume, by way of contradiction, that there is an index $j \in I_{n}$ with $\sigma_{i}(\mathrm{~L})=\sigma_{i}\left(\left\{\ell_{j}, \bar{\ell}_{j}\right\}\right)$. Set $i:=1$. Consider now player 2 switching to a literal $\ell$. By the utility functions (Cases (3) and (4)), player 2 gets utility 1 if and only if $\ell \in \mathrm{L}^{\prime}:=\left\{\ell_{j+2}, \bar{\ell}_{j+2}, \ell_{j+3}, \bar{\ell}_{j+3}\right\}$ and all non-special players choose $\delta$. By Lemma 5.3 (Condition (2)), for each non-special player $i^{\prime} \in[r] \backslash[2]$, $\delta \in \operatorname{Supp}\left(\sigma_{i^{\prime}}\right)$. Hence, there is at least one profile from Case (3) supported in $\sigma_{-2} \diamond \ell$. It follows that $\mathrm{U}_{2}\left(\boldsymbol{\sigma}_{-2} \diamond \ell_{k}\right)>\mathrm{U}_{2}\left(\boldsymbol{\sigma}_{-2} \diamond \ell_{h}\right)$ for any pair of literals $\ell_{k} \in \mathrm{L}^{\prime}$ and $\ell_{h} \notin \mathrm{L}^{\prime}$. By Lemma 2.1 (Condition (2)), this implies that $\operatorname{Supp}\left(\sigma_{2}\right) \cap \mathrm{L} \subseteq \mathrm{L}^{\prime}$.

Now, taking that $\operatorname{Supp}\left(\sigma_{2}\right) \cap \mathrm{L} \subseteq \mathrm{L}^{\prime}$, consider player 1 switching to a literal $\ell$. By the utility functions (Cases (1), (2) and (4)), if $\ell \notin \mathrm{L}^{\prime \prime}:=\left\{\ell_{j+1}, \bar{\ell}_{j+1}, \ell_{j+2}, \bar{\ell}_{j+2}, \ell_{j+3}, \bar{\ell}_{j+3}\right\}$ then player 1 gets utility 0 ; if she chooses some literal $\ell_{k} \in \mathrm{L}^{\prime \prime}$ and all non-special players choose $\delta$ then she gets utility 1 By Lemma $\$ 5.3$ (Condition (2)), for each non-special player $i^{\prime} \in[r] \backslash[2]$, $\delta \in \operatorname{Supp}\left(\sigma_{i^{\prime}}\right)$. Hence, there is at least one profile from Case (2) supported in $\sigma_{-1} \diamond \ell$. It follows that $\mathrm{U}_{1}\left(\boldsymbol{\sigma}_{-1} \diamond \ell_{k}\right)>\mathrm{U}_{1}\left(\boldsymbol{\sigma}_{-1} \diamond \ell_{h}\right)$ for any literal $\ell_{h} \notin \mathrm{L}^{\prime \prime}$. By Lemma 2.1 (Condition (2)), this implies that $\operatorname{Supp}\left(\sigma_{1}\right) \cap \mathbf{L} \subseteq \mathrm{L}^{\prime \prime}$. Note that with $n \geq 4$, this implies $\ell_{j} \notin \operatorname{Supp}\left(\sigma_{1}\right) \cap \mathbf{L}$. A contradiction. We use a corresponding argument to establish the claim for player 2 .

We continue to prove:

Claim 5.7 The following conditions hold for each index $k \in I_{n}$ :

(C.1) If $\sigma_{1}\left(\left\{\ell_{k}, \bar{\ell}_{k}\right\}\right)>0$ then $\sigma_{2}\left(\left\{\ell_{k}, \bar{\ell}_{k}\right\}\right) \cdot \sigma_{2}\left(\left\{\ell_{k+1}, \bar{\ell}_{k+1}\right\}\right)>0$.

(C.2) If $\sigma_{2}\left(\left\{\ell_{k}, \bar{\ell}_{k}\right\}\right)>0$ then $\sigma_{1}\left(\left\{\ell_{k-3}, \bar{\ell}_{k-3}\right\}\right) \cdot \sigma_{1}\left(\left\{\ell_{k-2}, \bar{\ell}_{k-2}\right\}\right)>0$.

Proof: We start with Condition (C.1). Claim 5.6 implies that there are at least two distinct indices $j, h \in I_{n}$ with $\sigma_{2}\left(\left\{\ell_{j}, \bar{\ell}_{j}\right\}\right)>0$ and $\sigma_{2}\left(\left\{\ell_{h}, \bar{\ell}_{h}\right\}\right)>0$. If $\{k, k+1\}=\{j, h\}$, then we are done. So assume that $\{k, k+1\} \neq\{j, h\}$. This implies that either $k \neq j$ and $k+1 \neq j$ or $k \neq h$ and $k+1 \neq h$. Choose $k \neq h$ and $k+1 \neq h$. 
Since $\sigma_{1}\left(\left\{\ell_{k}, \bar{\ell}_{k}\right\}\right)>0$, either $\ell_{k}$ or $\bar{\ell}_{k}$ is a best-response for player 1 to $\sigma_{-1}$; assume, without loss of generality, that $\ell_{k}$ is a best-response for player 1 to $\sigma_{-1}$. Consider player 1 switching to $\ell_{k}$. By the utility functions (Cases (1), (2) and (4)), player 1 gets utility 1 if and only if player 2 chooses a literal from $\left\{\ell_{k}, \ell_{k+1}, \bar{\ell}_{k+1}\right\}$ and all non-special players choose $\delta$. Hence,

$$
\mathrm{U}_{1}\left(\sigma_{-1} \diamond \ell_{k}\right)=\sigma_{2}\left(\left\{\ell_{k}, \ell_{k+1}, \bar{\ell}_{k+1}\right\}\right) \cdot \prod_{i \in[r] \backslash[2]} \sigma_{i}(\delta)
$$

Consider player 1 switching to $\mathrm{v}_{h, k}$. By the utility functions (Case (5)), player 1 gets utility 1 if and only if player 2 chooses a literal from $\left\{\ell_{h}, \bar{\ell}_{h}, \ell_{k}, \bar{\ell}_{k}\right\}$ and all non-special players choose $\delta$. Hence,

$$
\mathrm{U}_{1}\left(\boldsymbol{\sigma}_{-1} \diamond \mathrm{v}_{h, k}\right)=\sigma_{2}\left(\left\{\ell_{h}, \bar{\ell}_{h}, \ell_{k}, \bar{\ell}_{k}\right\}\right) \cdot \prod_{i \in[r] \backslash[2]} \sigma_{i}(\delta) .
$$

Since $\ell_{k}$ is a best-response for player 1 to $\sigma_{-1}$, it follows that

$$
\sigma_{2}\left(\left\{\ell_{k}, \ell_{k+1}, \bar{\ell}_{k+1}\right\}\right) \cdot \prod_{i \in[r] \backslash[2]} \sigma_{i}(\delta) \geq \sigma_{2}\left(\left\{\ell_{h}, \bar{\ell}_{h}, \ell_{k}, \bar{\ell}_{k}\right\}\right) \cdot \prod_{i \in[r] \backslash[2]} \sigma_{i}(\delta) .
$$

By Lemma 5.3 (Condition (2)), $\prod_{i \in[r] \backslash[2]} \sigma_{i}(\delta) \neq 0$; thus, it follows that

$$
\sigma_{2}\left(\left\{\ell_{k}, \ell_{k+1}, \bar{\ell}_{k+1}\right\}\right) \geq \sigma_{2}\left(\left\{\ell_{h}, \bar{\ell}_{h}, \ell_{k}, \bar{\ell}_{k}\right\}\right),
$$

which implies that

$$
\sigma_{2}\left(\left\{\ell_{k+1}, \bar{\ell}_{k+1}\right\}\right) \geq \sigma_{2}\left(\left\{\ell_{h}, \bar{\ell}_{h}, \bar{\ell}_{k}\right\}\right) .
$$

Since $\sigma_{2}\left(\left\{\ell_{h}, \bar{\ell}_{h},\right\}\right)>0$, it follows that $\sigma_{2}\left(\left\{\ell_{k+1}, \bar{\ell}_{k+1}\right\}\right)>0$.

Consider now player 1 switching to $v_{h, k+1}$. By the utility functions (Case (5)), player 1 gets utility 1 if and only if player 2 chooses a literal from $\left\{\ell_{h}, \bar{\ell}_{h}, \ell_{k+1}, \bar{\ell}_{k+1}\right\}$ and all non-special players choose $\delta$. Hence,

$$
\mathrm{U}_{1}\left(\boldsymbol{\sigma}_{-1} \diamond \mathrm{v}_{h, k+1}\right)=\sigma_{2}\left(\left\{\ell_{h}, \bar{\ell}_{h}, \ell_{k+1}, \bar{\ell}_{k+1}\right\}\right) \cdot \prod_{i \in[r] \backslash[2]} \sigma_{i}(\delta) .
$$

Since $\ell_{k}$ is a best-response for player 1 to $\sigma_{-1}$, it follows that

$$
\sigma_{2}\left(\left\{\ell_{k}, \ell_{k+1}, \bar{\ell}_{k+1}\right\}\right) \cdot \prod_{i \in[r] \backslash[2]} \sigma_{i}(\delta) \geq \sigma_{2}\left(\left\{\ell_{h}, \bar{\ell}_{h}, \ell_{k+1}, \bar{\ell}_{k+1}\right\}\right) \cdot \prod_{i \in[r] \backslash[2]} \sigma_{i}(\delta) .
$$

By Lemma 5.3 (Condition (2)), $\prod_{i \in[r] \backslash[2]} \sigma_{i}(\delta) \neq 0$; thus, it follows that

$$
\sigma_{2}\left(\left\{\ell_{k}, \ell_{k+1}, \bar{\ell}_{k+1}\right\}\right) \geq \sigma_{2}\left(\left\{\ell_{h}, \bar{\ell}_{h}, \ell_{k+1}, \bar{\ell}_{k+1}\right\}\right),
$$

which implies that

$$
\sigma_{2}\left(\left\{\ell_{k}, \bar{\ell}_{k}\right\}\right) \geq \sigma_{2}\left(\left\{\ell_{k}\right\}\right) \geq \sigma_{2}\left(\left\{\ell_{h}, \bar{\ell}_{h}\right\}\right) .
$$


Since $\sigma_{2}\left(\left\{\ell_{h}, \bar{\ell}_{h}\right\}\right)>0$, it follows that $\sigma_{2}\left(\left\{\ell_{k}, \bar{\ell}_{k}\right\}\right)>0$. This completes the proof of Condition (1).

We continue with Condition (C.2). Claim 5.6 implies that there are at least two distinct indices $j, h \in I_{n}$ with $\sigma_{1}\left(\left\{\ell_{j}, \bar{\ell}_{j}\right\}\right)>0$ and $\sigma_{1}\left(\left\{\ell_{h}, \bar{\ell}_{h}\right\}\right)>0$. If $\{k-3, k-2\}=\{j, h\}$, then we are done. So assume that $\{k-3, k-2\} \neq\{j, h\}$. This implies that either $k-3 \neq j$ and $k-2 \neq j$ or $k-3 \neq h$ and $k-2 \neq h$. Choose $k-3 \neq h$ and $k-2 \neq h$.

Since $\sigma_{2}\left(\left\{\ell_{k}, \bar{\ell}_{k}\right\}\right)>0$, either $\ell_{k}$ or $\bar{\ell}_{k}$ is a best-response for player 2 to $\boldsymbol{\sigma}_{-2}$; assume, without loss of generality, that $\ell_{k}$ is a best-response for player 2 to $\boldsymbol{\sigma}_{-2}$. Consider player 2 switching to $\ell_{k}$. By the utility functions (Cases (1), (2) and (4)), player 2 gets utility 1 if and only if player 1 chooses a literal from $\left\{\ell_{k-2}, \bar{\ell}_{k-2}, \ell_{k-3}, \bar{\ell}_{k-3}\right\}$ and all non-special players choose $\delta$. Hence,

$$
\mathrm{U}_{2}\left(\boldsymbol{\sigma}_{-2} \diamond \ell_{k}\right)=\sigma_{1}\left(\left\{\ell_{k-2}, \bar{\ell}_{k-2}, \ell_{k-3}, \bar{\ell}_{k-3}\right\}\right) \cdot \prod_{i \in[r] \backslash[2]} \sigma_{i}(\delta)
$$

Consider player 2 switching to $\mathrm{v}_{h, k-2}$. By the utility functions (Case (5)), player 2 gets utility 1 if and only if player 1 chooses a literal from $\left\{\ell_{h}, \bar{\ell}_{h}, \ell_{k-2}, \bar{\ell}_{k-2}\right\}$ and all non-special players choose $\delta$. Hence,

$$
\mathrm{U}_{2}\left(\boldsymbol{\sigma}_{-2} \diamond \mathrm{v}_{h, k-2}\right)=\sigma_{1}\left(\left\{\ell_{h}, \bar{\ell}_{h}, \ell_{k-2}, \bar{\ell}_{k-2}\right\}\right) \cdot \prod_{i \in[r] \backslash[2]} \sigma_{i}(\delta) .
$$

Since $\ell_{k}$ is a best-response for player 2 to $\sigma_{-2}$, it follows that

$$
\sigma_{1}\left(\left\{\ell_{k-2}, \bar{\ell}_{k-2}, \ell_{k-3}, \bar{\ell}_{k-3}\right\}\right) \cdot \prod_{i \in[r] \backslash[2]} \sigma_{i}(\delta) \geq \sigma_{1}\left(\left\{\ell_{h}, \bar{\ell}_{h}, \ell_{k-2}, \bar{\ell}_{k-2}\right\}\right) \cdot \prod_{i \in[r] \backslash[2]} \sigma_{i}(\delta) .
$$

By Lemma 5.3 (Condition (2)), $\prod_{i \in[r] \backslash[2]} \sigma_{i}(\delta) \neq 0$; thus, it follows that

$$
\sigma_{1}\left(\left\{\ell_{k-2}, \bar{\ell}_{k-2}, \ell_{k-3}, \bar{\ell}_{k-3}\right\}\right) \geq \sigma_{1}\left(\left\{\ell_{h}, \bar{\ell}_{h}, \ell_{k-2}, \bar{\ell}_{k-2}\right\}\right)
$$

which implies that

$$
\sigma_{1}\left(\left\{\ell_{k-3}, \bar{\ell}_{k-3}\right\}\right) \geq \sigma_{1}\left(\left\{\ell_{h}, \bar{\ell}_{h}\right\}\right) .
$$

Since $\sigma_{1}\left(\left\{\ell_{h}, \bar{\ell}_{h},\right\}\right)>0$, it follows that $\sigma_{1}\left(\left\{\ell_{k-3}, \bar{\ell}_{k-3}\right\}\right)>0$.

Consider now player 2 switching to $\mathrm{v}_{h, k-3}$. By the utility functions (Case (5)), player 2 gets utility 1 if and only if player 1 chooses a literal from $\left\{\ell_{h}, \bar{\ell}_{h}, \ell_{k-3}, \bar{\ell}_{k-3}\right\}$ and all non-special players choose $\delta$. Hence,

$$
\mathrm{U}_{2}\left(\boldsymbol{\sigma}_{-2} \diamond \mathrm{v}_{h, k-3}\right)=\sigma_{1}\left(\left\{\ell_{h}, \bar{\ell}_{h}, \ell_{k-3}, \bar{\ell}_{k-3}\right\}\right) \cdot \prod_{i \in[r] \backslash[2]} \sigma_{i}(\delta) .
$$

Since $\ell_{k}$ is a best-response for player 2 to $\sigma_{-2}$, it follows that

$$
\sigma_{1}\left(\left\{\ell_{k-2}, \bar{\ell}_{k-2}, \ell_{k-3}, \bar{\ell}_{k-3}\right\}\right) \cdot \prod_{i \in[r] \backslash[2]} \sigma_{i}(\delta) \geq \sigma_{1}\left(\left\{\ell_{h}, \bar{\ell}_{h}, \ell_{k-3}, \bar{\ell}_{k-3}\right\}\right) \cdot \prod_{i \in[r] \backslash[2]} \sigma_{i}(\delta) .
$$


By Lemma 5.3 (Condition (2)), $\prod_{i \in[r] \backslash[2]} \sigma_{i}(\delta) \neq 0$; thus, it follows that

$$
\sigma_{1}\left(\left\{\ell_{k-2}, \bar{\ell}_{k-2}, \ell_{k-3}, \bar{\ell}_{k-3}\right\}\right) \geq \sigma_{1}\left(\left\{\ell_{h}, \bar{\ell}_{h}, \ell_{k-3}, \bar{\ell}_{k-3}\right\}\right)
$$

which implies that

$$
\sigma_{1}\left(\left\{\ell_{k-2}, \bar{\ell}_{k-2}\right\}\right) \geq \sigma_{1}\left(\left\{\ell_{h}, \bar{\ell}_{h}\right\}\right) .
$$

Since $\sigma_{1}\left(\left\{\ell_{h}, \bar{\ell}_{h},\right\}\right)>0$, it follows that $\sigma_{1}\left(\left\{\ell_{k-2}, \bar{\ell}_{k-2}\right\}\right)>0$, and this completes the proof.

We continue to prove:

Claim 5.8 Fix a player $i \in[2]$. Then, for each index $j \in I_{n}, \sigma_{i}\left(\left\{\ell_{j}, \bar{\ell}_{j}\right\}\right)>0$.

Proof: Assume, by way of contradiction, that there is an index $j \in I_{n}$ with $\sigma_{i}\left(\left\{\ell_{j}, \bar{\ell}_{j}\right\}\right)=0$. Without loss of generality, take that $\sigma_{i}\left(\left\{\ell_{j+1}, \bar{\ell}_{j+1}\right\}\right)>0$. There are two cases: $(1) i=1$. By Claim 5.7 (Condition (C.1)), $\sigma_{2}\left(\left\{\ell_{j+2}, \bar{\ell}_{j+2}\right\}\right)>0$; hence, by Claim 5.7 (Condition (C.2)), $\sigma_{1}\left(\left\{\ell_{j}, \bar{\ell}_{j}\right\}\right)>0$. A contradiction. (2) $i=2$. By Claim 5.7(Condition (C.2)), $\sigma_{1}\left(\left\{\ell_{j-1}, \bar{\ell}_{j-1}\right\}\right)>$ 0 ; hence, by Claim 5.7 (Condition $\overline{(\mathrm{C} .1)), \sigma_{2}}\left(\left\{\ell_{j}, \bar{\ell}_{j}\right\}\right)>0$. A contradiction.

To prove that for each index $j \in I_{n}, \sigma_{i}\left(\left\{\ell_{j}, \bar{\ell}_{j}\right\}\right)=\frac{\sigma_{i}(\mathrm{~L})}{n}$, assume, by way of contradiction, that there is an index $j \in I_{n}$ with

$$
\sigma_{i}\left(\left\{\ell_{j}, \bar{\ell}_{j}\right\}\right)>\sigma_{i}\left(\left\{\ell_{j+1}, \bar{\ell}_{j+1}\right\}\right) .
$$

Fix now $i:=2$. We proceed by case analysis.

1. Assume first that there is an index $k \in I_{n} \backslash\{j, j+1\}$ with

$$
\sigma_{1}\left(\left\{\ell_{k}, \bar{\ell}_{k}\right\}\right)>\sigma_{1}\left(\left\{\ell_{j+1}, \bar{\ell}_{j+1}\right\}\right) .
$$

By Claim [5.8, either $\ell_{j+3}$ or $\bar{\ell}_{j+3}$ is a best-response for player 2 to $\boldsymbol{\sigma}_{-2}$; assume, without loss of generality, that $\ell_{j+3}$ is such a best-response. Consider first player 2 switching to $\ell_{j+3}$. By the utility functions (Cases (3) and (4)), player 2 gets utility 1 if and only if player 1 chooses a literal from $\left\{\ell_{j}, \bar{\ell}_{j}, \ell_{j+1}, \bar{\ell}_{j+1}\right\}$ and all non-special players choose $\delta$. Hence,

$$
\mathrm{U}_{2}\left(\boldsymbol{\sigma}_{-2} \diamond \ell_{j+3}\right)=\sigma_{1}\left(\left\{\ell_{j}, \bar{\ell}_{j}, \ell_{j+1}, \bar{\ell}_{j+1}\right\}\right) \cdot \prod_{i \in[r] \backslash[2]} \sigma_{i}(\delta)
$$

Consider now player 2 switching to the variable $\mathrm{v}_{j, k}$. By the utility functions (Case $(5 / b))$, player 2 gets utility 1 if and only if player 1 chooses a literal from $\left\{\ell_{j}, \bar{\ell}_{j}, \ell_{k}, \bar{\ell}_{k}\right\}$ and all non-special players choose $\delta$. Hence,

$$
\mathrm{U}_{2}\left(\boldsymbol{\sigma}_{-2} \diamond \mathrm{v}_{j, k}\right)=\sigma_{1}\left(\left\{\ell_{j}, \bar{\ell}_{j}, \ell_{k}, \bar{\ell}_{k}\right\}\right) \cdot \prod_{i \in[r] \backslash[2]} \sigma_{i}(\delta) .
$$


Since $\ell_{j+3}$ is a best-response for player 2 to $\sigma_{-2}$, it follows that

$$
\sigma_{1}\left(\left\{\ell_{j}, \bar{\ell}_{j}, \ell_{j+1}, \bar{\ell}_{j+1}\right\}\right) \cdot \prod_{i \in[r] \backslash[2]} \sigma_{i}(\delta) \geq \sigma_{1}\left(\left\{\ell_{j}, \bar{\ell}_{j}, \ell_{k}, \bar{\ell}_{k}\right\}\right) \cdot \prod_{i \in[r] \backslash[2]} \sigma_{i}(\delta) .
$$

By Lemma 5.3 (Condition (2)), $\prod_{i \in[r] \backslash[2]} \sigma_{i}(\delta) \neq 0$; thus, it follows that

$$
\sigma_{1}\left(\left\{\ell_{j}, \bar{\ell}_{j}, \ell_{j+1}, \bar{\ell}_{j+1}\right\}\right) \geq \sigma_{1}\left(\left\{\ell_{j}, \bar{\ell}_{j}, \ell_{k}, \bar{\ell}_{k}\right\}\right),
$$

which implies that

$$
\sigma_{1}\left(\left\{\ell_{j+1}, \bar{\ell}_{j+1}\right\}\right) \geq \sigma_{1}\left(\left\{\ell_{k}, \bar{\ell}_{k}\right\}\right)
$$

A contradiction.

2. Assume now that there is no index $k \in I_{n} \backslash\{j, j+1\}$ with

$$
\sigma_{1}\left(\left\{\ell_{k}, \bar{\ell}_{k}\right\}\right)>\sigma_{1}\left(\left\{\ell_{j+1}, \bar{\ell}_{j+1}\right\}\right) \text {. }
$$

It follows that for each index $k \in I_{n} \backslash\{j, j+1\}$,

$$
\sigma_{1}\left(\left\{\ell_{k}, \bar{\ell}_{k}\right\}\right) \leq \sigma_{1}\left(\left\{\ell_{j+1}, \bar{\ell}_{j+1}\right\}\right) \text {. }
$$

Choose $k:=j+2$; so,

$$
\sigma_{1}\left(\left\{\ell_{j+2}, \bar{\ell}_{j+2}\right\}\right) \leq \sigma_{1}\left(\left\{\ell_{j+1}, \bar{\ell}_{j+1}\right\}\right) .
$$

Consider first player 2 switching to $\ell_{j+4}$. By the utility functions (Cases (3) and (4)), player 2 gets utility 1 if and only if player 1 chooses a literal from $\left\{\ell_{j+1}, \bar{\ell}_{j+1}, \ell_{j+2}, \bar{\ell}_{j+2}\right\}$ and all non-special players choose $\delta$. Hence,

$$
\mathrm{U}_{2}\left(\boldsymbol{\sigma}_{-2} \diamond \ell_{j+4}\right)=\sigma_{1}\left(\left\{\ell_{j+1}, \bar{\ell}_{j+1}, \ell_{j+2}, \bar{\ell}_{j+2}\right\}\right) \cdot \prod_{i \in[r] \backslash[2]} \sigma_{i}(\delta) .
$$

Consider now player 2 switching to the variable $v_{j, j+1}$. By the utility functions (Case (5)), player 2 gets utility 1 if and only if player 1 chooses a literal from $\left\{\ell_{j}, \bar{\ell}_{j}, \ell_{j+1}, \bar{\ell}_{j+1}\right\}$. and all non-special players choose $\delta$. Hence,

$$
\mathrm{U}_{2}\left(\boldsymbol{\sigma}_{-2} \diamond \mathrm{v}_{j, j+1}\right)=\sigma_{1}\left(\left\{\ell_{j}, \bar{\ell}_{j}, \ell_{j+1}, \bar{\ell}_{j+1}\right\}\right) \cdot \prod_{i \in[r] \backslash[2]} \sigma_{i}(\delta) .
$$

Since $\ell_{j}$ is a best-response for player 1 to $\sigma_{-1}$, it follows that

$$
\sigma_{1}\left(\left\{\ell_{j+1}, \bar{\ell}_{j+1}, \ell_{j+2}, \bar{\ell}_{j+2}\right\}\right) \cdot \prod_{i \in[r] \backslash[2]} \sigma_{i}(\delta) \geq \sigma_{1}\left(\left\{\ell_{j}, \bar{\ell}_{j}, \ell_{J+1}, \bar{\ell}_{j+1}\right\}\right) \cdot \prod_{i \in[r] \backslash[2]} \sigma_{i}(\delta) .
$$

By Lemma 5.3 (Condition (2)), $\prod_{i \in[r] \backslash[2]} \sigma_{i}(\delta) \neq 0$; hence, it follows that

$$
\sigma_{1}\left(\left\{\ell_{j+1}, \bar{\ell}_{+1 j}, \ell_{j+2}, \bar{\ell}_{j+2}\right\}\right) \geq \sigma_{1}\left(\left\{\ell_{j}, \bar{\ell}_{j}, \ell_{j+1}, \bar{\ell}_{j+1}\right\}\right),
$$

which implies that

$$
\sigma_{1}\left(\left\{\ell_{j+2}, \bar{\ell}_{j+2}\right\}\right) \geq \sigma_{1}\left(\left\{\ell_{j}, \bar{\ell}_{j}\right\}\right) .
$$


It follows that

$$
\sigma_{1}\left(\left\{\ell_{j+1}, \bar{\ell}_{j+1}\right\}\right) \geq \sigma_{1}\left(\left\{\ell_{j}, \bar{\ell}_{j}\right\}\right)
$$

A contradiction.

We use a corresponding argument to establish the claim when $i=1$.

We prove that in a Nash equilibrium from $\mathcal{N} \mathcal{E}(\mathrm{G}) \backslash \mathcal{N} \mathcal{E}(\widehat{\mathrm{G}})$, each non-special player chooses $\delta$.

Lemma 5.9 Fix a Nash equilibrium $\boldsymbol{\sigma} \in \mathcal{N} \mathcal{E}(\mathrm{G}) \backslash \mathcal{N} \mathcal{E}(\widehat{\mathrm{G}})$. Then, for each player $i \in[r] \backslash[2]$, $\sigma_{i}(\delta)=1$.

Proof: Assume, by way of contradiction, that there is a player $i \in[r] \backslash[2]$ with $\sigma_{i}(\delta)<1$. Since $\Sigma_{i}=\widehat{\Sigma}_{i} \cup\{\delta\}$, this implies that $\sigma_{i}\left(\widehat{\Sigma}_{i}\right)>0$. Lemma 5.3 (Condition (2)) implies that $\sigma_{i}\left(\widehat{\Sigma}_{i}\right)<1$. Since $\Sigma_{i}=\widehat{\Sigma}_{i} \cup\{\delta\}$, it follows that $\sigma_{i}(\delta)>0$.

By Lemma 5.4, there is a literal $\ell_{j} \in \operatorname{Supp}\left(\sigma_{1}\right)$. Consider player 1 switching to $\ell_{j}$. By the utility functions, player 1 gets utility 1 if and only if player 2 chooses a literal from $\left\{\ell_{j}, \ell_{j+1}, \bar{\ell}_{j+1}\right\}$ and each non-special player chooses $\delta$ (Cases (1), (2) and (4)). Hence,

$$
\begin{aligned}
& \mathrm{U}_{1}\left(\sigma_{-1} \diamond \ell_{j}\right) \\
& =\prod_{i^{\prime} \notin[2] \cup\{i\}} \sigma_{i^{\prime}}(\delta) \cdot \sigma_{i}(\delta) \cdot \sigma_{2}\left(\left\{\ell_{j}, \ell_{j+1}, \bar{\ell}_{j+1}\right\}\right) \\
& \leq \quad \sigma_{i}(\delta) \cdot 2 \cdot \frac{\sigma_{2}(\mathrm{~L})}{n} \quad \text { (by Lemma 5.5) } \\
& <\quad 2 \cdot \frac{\sigma_{2}(\mathrm{~L})}{n} \quad\left(\text { since } \sigma_{i}(\delta)<1\right) \text {. }
\end{aligned}
$$

Consider now player 1 switching to a strategy $s \in \widehat{\Sigma}_{1}$. By the utility functions, player 1 gets utility 1 if player 2 chooses a literal from $\left\{\ell_{0}, \bar{\ell}_{0}, \ell_{1}, \bar{\ell}_{1}\right\}$ (Case (10)). Then, by Lemma 5.5.

$$
\mathrm{U}_{1}\left(\sigma_{-1} \diamond s\right) \geq 2 \cdot \frac{\sigma_{2}(\mathrm{~L})}{n} .
$$

By Lemma 5.4, $\sigma_{2}(\mathrm{~L})>0$. This implies that

$$
\mathrm{U}_{1}\left(\boldsymbol{\sigma}_{-1} \diamond s\right)>\mathrm{U}_{1}\left(\boldsymbol{\sigma}_{-1} \diamond \ell_{j}\right)
$$

Since $\boldsymbol{\sigma}$ is a Nash equilibrium and $\ell_{j} \in \operatorname{Supp}\left(\sigma_{1}\right)$, Lemma 2.1 (Condition (2)) implies that

$$
\mathrm{U}_{1}\left(\boldsymbol{\sigma}_{-1} \diamond s\right) \leq \mathrm{U}_{1}\left(\boldsymbol{\sigma}_{-1} \diamond \ell_{j}\right)
$$

A contradiction. 
Lemma 5.9 implies that for a Nash equilibrium $\sigma \in \mathcal{N E}(\mathrm{G}) \backslash \mathcal{N} \mathcal{E}(\widehat{\mathrm{G}})$, the utility of each special player $i \in[2]$ in either $\boldsymbol{\sigma}$ or $\boldsymbol{\sigma}_{-i} \diamond s$, where $s \in \Sigma_{i}$, is solely determined by the strategies chosen by the two special players. We prove that each special player is exclusively playing literals.

Lemma 5.10 (Special Players Only Play Literals) Fix a Nash equilibrium $\boldsymbol{\sigma} \in \mathcal{N} \mathcal{E}(\mathrm{G}) \backslash$ $\mathcal{N E}(\widehat{\mathrm{G}})$ and a special player $i \in[2]$. Then, $\sigma_{i}(\mathrm{~L})=1$.

Proof: By Lemma [5.4, there is a literal $\ell_{j} \in \operatorname{Supp}\left(\sigma_{\bar{i}}\right)$. Consider player $\bar{i}$ switching to $\ell_{j}$. By the utility functions, Lemma 5.9 implies that player $\bar{i}$ gets utility 1 if and only if player $i$ chooses a literal from $\left\{\ell_{j}, \ell_{j+1}, \bar{\ell}_{j+1}\right\}$ (Cases (1), (2) and (4), with $i=2$ ) (resp., player $i$ chooses a literal from $\left\{\ell_{j-2}, \bar{\ell}_{j-2}, \ell_{j-3}, \bar{\ell}_{j-3}\right\}$ (Cases (3) and (4), with $\left.i=1\right)$ ). Hence, for $i=2$,

$$
\mathrm{U}_{\bar{i}}\left(\boldsymbol{\sigma}_{-\bar{i}} \diamond \ell_{j}\right)=\sigma_{i}\left(\left\{\ell_{j}, \ell_{j+1}, \bar{\ell}_{j+1}\right\}\right) \text {, }
$$

while for $i=1$,

$$
\mathrm{U}_{\bar{i}}\left(\sigma_{-\bar{i}} \diamond \ell_{j}\right)=\sigma_{i}\left(\left\{\ell_{j-2}, \bar{\ell}_{j-2}, \ell_{j-3}, \bar{\ell}_{j-3}\right\}\right),
$$

By Lemma 5.5, these imply that

$$
\mathrm{U}_{\bar{i}}\left(\sigma_{-\bar{i}} \diamond \ell_{j}\right) \leq 2 \cdot \frac{\sigma_{i}(\mathrm{~L})}{n} .
$$

Consider now player $\bar{i}$ switching to a strategy $s \in \widehat{\Sigma}_{\bar{i}}$.

Assume, by way of contradiction, that $\sigma_{i}(\mathcal{C} \cup \mathrm{V})>0$. By the utility functions, player $\bar{i}$ gets utility 1 if player $i$ chooses a strategy $s \in\left\{\ell_{0}, \bar{\ell}_{0}, \ell_{1}, \bar{\ell}_{1}\right\} \cup \mathcal{C} \cup \vee($ Case (10)). Then,

$$
\begin{aligned}
& \mathrm{U}_{\bar{i}}\left(\boldsymbol{\sigma}_{-i} \diamond s\right) \geq \sigma_{i}\left(\left\{\ell_{0}, \bar{\ell}_{0}, \ell_{1}, \bar{\ell}_{1}\right\}\right)+\sigma_{i}(\mathcal{C} \cup \mathrm{V}) \\
& >\quad \sigma_{i}\left(\left\{\ell_{0}, \bar{\ell}_{0}, \ell_{1}, \bar{\ell}_{1}\right\}\right) \quad\left(\text { since } \sigma_{i}(\mathcal{C}, \mathrm{V})>0\right) \\
& \geq \quad 2 \cdot \frac{\sigma_{2}(\mathrm{~L})}{n} \text {. } \quad \text { (by Lemma } 5.5 \text {. }
\end{aligned}
$$

By Lemma 5.4, $\sigma_{2}(\mathrm{~L})>0$. This implies that

$$
\mathrm{U}_{\bar{i}}\left(\sigma_{-\bar{i}} \diamond s\right)>\mathrm{U}_{\bar{i}}\left(\sigma_{-\bar{i}} \diamond \ell_{j}\right)
$$

Since $\boldsymbol{\sigma}$ is a Nash equilibrium and $\ell_{j} \in \operatorname{Supp}\left(\sigma_{\bar{i}}\right)$, Lemma 2.1 (Condition (2)) implies that

$$
\mathrm{U}_{\bar{i}}\left(\boldsymbol{\sigma}_{-\bar{i}} \diamond s\right) \leq \mathrm{U}_{\bar{i}}\left(\boldsymbol{\sigma}_{-\bar{i}} \diamond \ell_{j}\right) .
$$

A contradiction. It follows that $\sigma_{i}(\mathcal{C} \cup \mathrm{V})=0$.

Assume, by way of contradiction, that $\sigma_{i}\left(\widehat{\Sigma}_{i}\right)>0$. By the utility functions, player $\bar{i}$ gets utility 1 if player $i$ chooses a strategy $s \in\left\{\ell_{0}, \bar{\ell}_{0}, \ell_{1}, \bar{\ell}_{1}\right\} \cup \widehat{\Sigma}_{i}$ (Cases (8) and (10)). Then,

$$
\begin{aligned}
& \mathrm{U}_{\bar{i}}\left(\boldsymbol{\sigma}_{-i} \diamond s\right) \geq \sigma_{i}\left(\left\{\ell_{0}, \bar{\ell}_{0}, \ell_{1}, \bar{\ell}_{1}\right\}\right)+\sigma_{i}\left(\widehat{\Sigma}_{i}\right) \\
& >\quad \sigma_{i}\left(\left\{\ell_{0}, \bar{\ell}_{0}, \ell_{1}, \bar{\ell}_{1}\right\}\right) \quad\left(\text { since } \sigma_{i}\left(\widehat{\Sigma}_{i}\right)>0\right) \\
& \geq \quad 2 \cdot \frac{\sigma_{2}(\mathrm{~L})}{n} \text {. } \quad \text { (by Lemma 5.5). }
\end{aligned}
$$


By Lemma [5.4, $\sigma_{2}(\mathrm{~L})>0$. This implies that

$$
\mathrm{U}_{\bar{i}}\left(\boldsymbol{\sigma}_{-\bar{i}} \diamond s\right)>\mathrm{U}_{\bar{i}}\left(\boldsymbol{\sigma}_{-\bar{i}} \diamond \ell_{j}\right)
$$

Since $\boldsymbol{\sigma}$ is a Nash equilibrium and $\ell_{j} \in \operatorname{Supp}\left(\sigma_{\bar{i}}\right)$, Lemma 2.1 (Condition (2)) implies that

$$
\mathrm{U}_{\bar{i}}\left(\sigma_{-\bar{i}} \diamond s\right) \leq \mathrm{U}_{\bar{i}}\left(\sigma_{-\bar{i}} \diamond \ell_{j}\right)
$$

A contradiction. It follows that $\sigma_{i}\left(\widehat{\Sigma}_{i}\right)=0$.

Since $\sigma_{i}(\mathcal{C} \cup \mathbf{V})=\sigma_{i}\left(\widehat{\Sigma}_{i}\right)=0$, it follows that $\sigma_{i}(\mathrm{~L})=1$, and we are done.

In view of Lemma 5.10, a Nash equilibrium $\sigma \in \mathcal{N} \mathcal{E}(\mathrm{G}) \backslash \mathcal{N} \mathcal{E}(\widehat{\mathrm{G}})$ will be called a literal equilibrium. The next property of such literal equilibria is that the two special players are playing literals in a consistent way: for each literal, the two special players are both playing either the literal or its negation; this will imply that a literal equilibrium induces an assignment for $\phi$.

Lemma 5.11 Fix a Nash equilibrium $\boldsymbol{\sigma} \in \mathcal{N} \mathcal{E}(\mathrm{G}) \backslash \mathcal{N E}(\widehat{\mathrm{G}})$ and a literal $\ell \in \mathrm{L}$. Then, $\sigma_{1}(\ell)$. $\sigma_{2}(\bar{\ell})=0$.

Proof: Lemmas 5.5 and 5.10 imply together that for each player $i \in[2]$ and for each literal $\ell_{j} \in \mathrm{L}, \sigma_{i}\left(\left\{\ell_{j}, \bar{\ell}_{j}\right\}\right)=\frac{1}{n}$. Assume, by way of contradiction, that $\sigma_{1}\left(\ell_{j}\right) \cdot \sigma_{2}\left(\bar{\ell}_{j}\right)>0$. This implies that $\sigma_{2}\left(\ell_{j}\right)<\frac{1}{n}$. Hence, by the utility functions (Cases (1), (2) and (4)),

$$
\begin{array}{rlrl} 
& \mathrm{U}_{1}(\boldsymbol{\sigma}) & & \\
= & \mathrm{U}_{1}\left(\boldsymbol{\sigma} \diamond \ell_{j}\right) & \text { (by Lemma } 2.1(\text { Condition }(1))) \\
= & \sigma_{2}\left(\ell_{j}\right)+\sigma_{2}\left(\left\{\ell_{j+1}, \bar{\ell}_{j+1}\right\}\right) & \\
< & \frac{2}{n} . &
\end{array}
$$

Consider now player 1 switching to a strategy $s \in \widehat{\Sigma}_{1}$. Then, by the utility functions (Case (10)), player 1 gets utility 1 if player 2 chooses a literal from $\left\{\ell_{0}, \bar{\ell}_{0}, \ell_{1}, \bar{\ell}_{1}\right\}$. So,

$$
\mathrm{U}_{1}\left(\boldsymbol{\sigma}_{-1} \diamond s\right) \geq \sigma_{2}\left(\left\{\ell_{0}, \bar{\ell}_{0}, \ell_{1}, \bar{\ell}_{1}\right\}\right)=\frac{2}{n} .
$$

A contradiction.

We finally conclude:

Corollary 5.12 Fix a Nash equilibrium $\boldsymbol{\sigma} \in \mathcal{N} \mathcal{E}(\mathrm{G}) \backslash \mathcal{N} \mathcal{E}(\widehat{\mathrm{G}})$. Then, the following conditions hold:

(C.1) $\boldsymbol{\sigma}$ induces an assignment $\gamma(\boldsymbol{\sigma})$ for $\phi$. 
(C.2) For each player $i \in[2], \sigma_{i}$ is a uniform distribution on $\gamma(\boldsymbol{\sigma})$.

(C.3) $\mathrm{U}_{1}(\boldsymbol{\sigma})=\mathrm{U}_{2}(\boldsymbol{\sigma})=\frac{2}{n}$.

Proof: Condition (C.1) follows from Lemma 5.11. Condition (C.2) follows from Condition (C.1) and Lemma 5.5. Condition (C.3) follows from Condition (C.2) and the utility functions (Cases (2), (3) and (4)).

\subsection{Proof of the Reduction}

We are now ready to prove:

Proposition 5.13 Assume that $\phi$ is unsatisfiable. Then, $\mathcal{N E}(\mathrm{G})=\mathcal{N} \mathcal{E}(\widehat{\mathrm{G}})$.

We prepare the reader that the property that each clause c of $\phi$ contains $K \geq 3$ literals is necessary for the proof in order to render the deviation to c profitable in a Nash equilibrium. The assumption that $\phi$ is a 3SAT formula guarantees this property.

Proof: By Lemma 5.3 (Condition $(C .1)), \mathcal{N E}(\widehat{G}) \subseteq \mathcal{N} \mathcal{E}(G)$. So, assume, by way of contradiction, that there is a Nash equilibrium $\sigma \in \mathcal{N} \mathcal{E}(\mathrm{G}) \backslash \mathcal{N} \mathcal{E}(\widehat{\mathrm{G}})$. By Corollary 5.12 (Conditions (C.1) and (C.3)), $\boldsymbol{\sigma}$ induces an assignment $\gamma$ for $\phi$, and for each player $i \in[2], \mathrm{U}_{i}(\boldsymbol{\sigma})=\frac{2}{n}$. Denote as c the clause not satisfied by $\gamma$. Consider a special player $i \in[2]$ switching to c. Since $\phi$ is a 3SAT formula, there are three literals in c; for each such literal $\ell$, Corollary 5.12 (Condition (C.2)) implies that $\sigma_{\bar{i}}(\bar{\ell})=\frac{1}{n}$. Thus, $\mathbf{U}_{i}\left(\boldsymbol{\sigma}_{-i} \diamond \mathrm{c}\right)=\frac{3}{n}$. So, $\mathrm{U}_{i}\left(\boldsymbol{\sigma}_{-i} \diamond \mathrm{c}\right)>\mathrm{U}_{i}(\boldsymbol{\sigma})$. A contradiction to Lemma 2.1 (Condition (2)).

We continue to prove:

Proposition 5.14 Assume that $\phi$ is satisfiable. Then, for each satisfying assignment $\gamma, \mathrm{G}$ has a symmetric Nash equilibrium $\boldsymbol{\sigma}=\boldsymbol{\sigma}(\gamma) \in \mathcal{N} \mathcal{E}(\mathrm{G}) \backslash \mathcal{N} \mathcal{E}(\widehat{\mathrm{G}})$ such that for each player $i \in[2]$ :

(C.1) $\left|\operatorname{Supp}\left(\sigma_{i}\right)\right|=n$.

(C.2) $\mathrm{U}_{i}(\boldsymbol{\sigma})=\frac{2}{n}$

(C.3) $\operatorname{Supp}\left(\sigma_{i}\right) \cap \widehat{\Sigma}_{i}=\emptyset$.

(C.4) For each literal $\ell \in \mathrm{L}$ set to true by $\gamma, \ell \in \operatorname{Supp}\left(\sigma_{i}\right)$ with $\sigma_{i}(\ell)=\frac{1}{n}$. 
We prepare the reader that the property that each clause c of $\phi$ contains $K \leq 3$ literals is necessary for the proof in order to render the deviation of a player to c non-profitable in a Nash equilibrium. The assumption that $\phi$ is a 3SAT formula guarantees this property. Clearly, the assumption that $\phi$ is a 3SAT formula is the unique assumption that simultaneously guarantees both this property and the property needed for the proof of Proposition 5.13 .

Proof: Construct the mixed profile $\boldsymbol{\sigma}$ where, for each special player $i \in[2]$, for each literal $\ell \in \mathrm{L}$ set to true by $\gamma, \sigma_{i}(\ell):=\frac{1}{n}$; for each non-special player $i \in[r] \backslash[2], \sigma_{i}(\delta):=1$. Clearly, Conditions (C.1), (C.3) and (C.4) hold by construction; Condition (C.2) holds by the utility functions (Cases (2), (3) and (4)). It remains to prove that $\boldsymbol{\sigma}$ is a Nash equilibrium. There are four possible deviations for a special player $i \in[2]$ :

- To a strategy $s \in \widehat{\Sigma}$ : By the utility functions (Case (10)), player $i$ gets utility 1 for two possible choices of literals by player $\bar{i}$ : $\ell_{0}$ (resp., $\left.\bar{\ell}_{0}\right)$ and $\ell_{1}\left(\right.$ resp., $\left.\bar{\ell}_{1}\right)$. Hence, $\cup_{i}\left(\boldsymbol{\sigma}_{-i} \diamond s\right)=$ $\frac{2}{n}$.

- To a literal $\ell \in \mathrm{L}$ : By the utility functions (Cases (1), (2), (3) and (4)), player $i$ gets utility 1 for at most two possible choices of literals by player $\bar{i}$. Hence, $\bigcup_{i}\left(\boldsymbol{\sigma}_{-i} \diamond s\right) \leq \frac{2}{n}$.

- To a strategy $\mathrm{v}_{j, k} \in \mathrm{V}$ : By the utility functions (Case (5)), player $i$ gets utility 1 for two possible choices of literals by player $\bar{i}: \ell_{j}$ or $\bar{\ell}_{j}$, and $\ell_{k}$ or $\bar{\ell}_{k}$. Hence, $\mathrm{U}_{i}\left(\boldsymbol{\sigma}_{-i} \diamond \mathrm{v}_{j, k}\right)=\frac{2}{n}$.

- To a clause $c=\left\{\ell, \ell^{\prime}, \ell^{\prime \prime}\right\} \in \mathcal{C}$ : Since $\gamma$ is a satisfying assignment, $\gamma$ satisfies c. So, at least one of the literals from $\left\{\ell, \ell^{\prime}, \ell^{\prime \prime}\right\}$ is in $\gamma(\boldsymbol{\sigma})$. Since $\phi$ is a 3SAT formula, this implies that at most two of the literals from $\left\{\bar{\ell}, \bar{\ell}^{\prime}, \bar{\ell}^{\prime \prime}\right\}$ are not in $\gamma(\boldsymbol{\sigma})$. Hence, by construction, $\sigma_{\bar{i}}\left(\left\{\bar{\ell}, \bar{\ell}^{\prime}, \bar{\ell}^{\prime \prime}\right\}\right) \leq \frac{2}{n}$. By the utility functions (Case (6)), player $i$ gets utility 1 if and only if player $\bar{i}$ chooses a literal from $\left\{\bar{\ell}, \bar{\ell}^{\prime}, \bar{\ell}^{\prime \prime}\right\}$. It follows that $\mathrm{U}_{i}\left(\boldsymbol{\sigma}_{-i} \diamond \mathrm{c}\right) \leq \frac{2}{n}$.

Hence, player $i \in[2]$ cannot improve by switching. Consider now a non-special player $i \in[r] \backslash[2]$. By construction, $\sigma_{i}(\delta)=1$; hence, it follows, by the utility functions (Cases (2), (3) and (4)), that $\mathrm{U}_{i}(\boldsymbol{\sigma})=1$, and player $i$ cannot improve by switching. So $\boldsymbol{\sigma}$ is a Nash equilibrium.

Fix a win-lose game $\widehat{G}$ with the positive utility property. By Propositions 5.13 and $5.14, \mathrm{G}(\widehat{\mathrm{G}}, \phi)$ and $\widehat{G}$ are Nash-equivalent if and only if $\phi$ is unsatisfiable. So 3SAT reduces in polynomial time to $\overline{\mathrm{NASH}-\mathrm{EQUIVALENCE}(\widehat{\mathrm{G}})}$, so that, restricted to win-lose games, NASH-EQUIVALENCE $(\widehat{\mathrm{G}})$ is co- $\mathcal{N} \mathcal{P}$-hard. We shall later strengthen this co- $\mathcal{N} \mathcal{P}$-hardness result to the more restricted class of symmetric win-lose bimatrix games (Theorem 7.7). By Propositions 5.13 and 5.14 ,

$$
|\mathcal{N E}(\mathrm{G})|=|\mathcal{N} \mathcal{E}(\widehat{\mathrm{G}})|+\# \phi .
$$


Note that $|\mathcal{N E}(\widehat{G})|$ is a fixed constant when $\widehat{G}$ is independent of $\phi$. Since computing \# $\phi$ (resp., $\oplus \phi$ ) for a 3SAT formula $\phi$ is \#P्P-hard [35] (resp., $\oplus \mathcal{P}$-hard [33]), it follows that computing the number (resp., the parity of the number) of Nash equilibria for a win-lose bimatrix game is $\# \mathcal{P}$-hard (resp., $\oplus \mathcal{P}$-hard). We shall later strengthen the $\# \mathcal{P}$-hardness and the $\oplus \mathcal{P}$-hardness to the more restricted class of symmetric win-lose bimatrix games (Theorem 6.8).

\section{The Win-Lose GHR-Symmetrization}

For a given integer $n \geq 2$, denote as $0^{n}$ the $n$-dimensional null function $0^{n}:[n] \rightarrow\{0\}$; it is represented as the $n$-dimensional null vector in the natural way. Denote as $0^{n \times n}$ the $n \times n$ null matrix. For a positive $n$-dimensional vector $\boldsymbol{\rho} \in[0,1]^{n}$, denote as $\varrho$ the normalization of $\boldsymbol{\rho}$ to a probability vector; so, for each index $j \in[n], \varrho(j)=\frac{\rho(j)}{\sum_{k \in[n]} \rho(k)}$.

Given the bimatrix game $\mathrm{G}=\langle\mathrm{R}, \mathrm{C}\rangle$ and two functions $\mathrm{f}, \mathrm{g}:[n] \rightarrow \mathbb{R}$, define

$$
\overline{\mathrm{U}}_{1}(\mathrm{f}, \mathrm{g}):=\sum_{s_{1}, s_{2} \in[n]} \mathrm{f}\left(s_{1}\right) \cdot \mathrm{g}\left(s_{2}\right) \cdot \mathrm{R}\left[s_{1}, s_{2}\right]
$$

and

$$
\overline{\mathrm{U}}_{2}(\mathrm{f}, \mathrm{g}):=\sum_{s_{1}, s_{2} \in[n]} \mathrm{f}\left(s_{1}\right) \cdot \mathrm{g}\left(s_{2}\right) \cdot \mathrm{C}\left[s_{1}, s_{2}\right]
$$

So, $\overline{\mathrm{U}}_{1}(\mathrm{f}, \mathrm{g})$ and $\overline{\mathrm{U}}_{2}(\mathrm{f}, \mathrm{g})$ become the expected utilities of players 1 and 2 , respectively, in $\mathrm{G}$ when $f$ and $g$ are mixed strategies.

\subsection{Definition}

Definition 6.1 The GHR-symmetrization [21] of the bimatrix game $\langle\mathrm{R}, \mathrm{C}\rangle$, denoted as $\mathrm{GHR}(\langle\mathrm{R}, \mathrm{C}\rangle)$, is the symmetric bimatrix game $\left\langle\mathrm{S}, \mathrm{S}^{\mathrm{T}}\right\rangle$, where $\mathrm{S}$ is the $2 n \times 2 n$ matrix

$$
\mathrm{S}=\left(\begin{array}{cc}
0^{n \times n} & \mathrm{R} \\
\mathrm{C}^{\mathrm{T}} & 0^{n \times n}
\end{array}\right)
$$

so,

$$
\mathrm{S}^{\mathrm{T}}=\left(\begin{array}{cc}
0^{n \times n} & \mathrm{C} \\
\mathrm{R}^{\mathrm{T}} & 0^{n \times n}
\end{array}\right) .
$$

Note that the GHR-symmetrization of $\langle\mathrm{R}, \mathrm{C}\rangle$ is polynomial time computable. For shorter notation, denote $\widetilde{G}:=\operatorname{GHR}(\langle R, C\rangle)$. Clearly, when $\langle R, C\rangle$ is a win-lose game, so also is $G H R(\langle R, C\rangle)$. To emphasize this property, we shall term the GHR-symmetrization as the win-lose GHRsymmetrization. We further observe: 


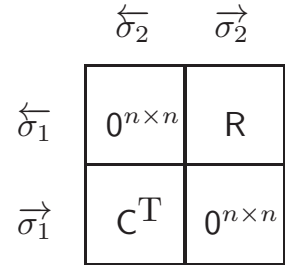

S

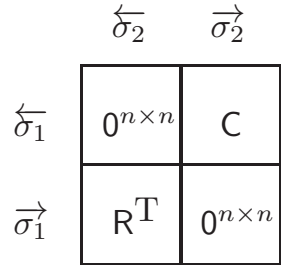

$\mathrm{s}^{\mathrm{T}}$

Figure 3: The bimatrix game $\widetilde{\mathrm{G}}=\left\langle\mathrm{S}, \mathrm{S}^{\mathrm{T}}\right\rangle$ with the mixed profile $\boldsymbol{\sigma}$.

Lemma 6.1 The win-lose GHR-symmetrization preserves the positive utility property.

Given a mixed profile $\boldsymbol{\sigma}=\left\langle\sigma_{1}, \sigma_{2}\right\rangle$ for $\widetilde{\mathrm{G}}$, denote, for each player $i \in[2]$, as $\overrightarrow{\sigma_{i}}$ (resp., $\overleftarrow{\sigma_{i}}$ ) the restriction of $\sigma_{i}$ to the last (resp., first) $n$ strategies of player $i$, so that for each strategy $j \in[n]$, $\overleftarrow{\sigma_{i}}(j)=\sigma_{i}(j)$ and $\overrightarrow{\sigma_{i}}(j)=\sigma_{i}(n+j)$. Thus, $\sigma_{i}=\overleftarrow{\sigma_{i}} \circ \overrightarrow{\sigma_{i}}$ is the concatenation of $\overleftarrow{\sigma_{i}}$ and $\overrightarrow{\sigma_{i}}$, which are its first and second component, respectively; $\boldsymbol{\sigma}=\left\langle\overleftarrow{\sigma_{1}} \circ \overrightarrow{\sigma_{1}}, \overleftarrow{\sigma_{2}} \circ \overrightarrow{\sigma_{2}}\right\rangle$, where $\overleftarrow{\sigma_{1}} \circ \overrightarrow{\sigma_{1}}$ and $\overleftarrow{\sigma_{2}} \circ \overrightarrow{\sigma_{2}}$ are the first and second entry, respectively, of $\boldsymbol{\sigma}$. See Figure 3 for a pictorial representation of the components $\overleftarrow{\sigma_{1}}$ and $\overrightarrow{\sigma_{1}}$ (resp., $\overleftarrow{\sigma_{2}}$ and $\overrightarrow{\sigma_{2}}$ ) and their association with the blocks $\mathrm{R}$ and $\mathrm{C}^{\mathrm{T}}$ (resp., $\mathrm{C}$ and $\mathrm{R}^{\mathrm{T}}$ ) of $\mathrm{S}$ (resp., $\mathrm{S}^{\mathrm{T}}$ ). We observe:

Observation 6.1 The expected utilities $\widetilde{\mathrm{U}}_{1}(\boldsymbol{\sigma})$ and $\widetilde{\mathrm{U}}_{2}(\boldsymbol{\sigma})$ are:

(Eq. 1) $\widetilde{\mathrm{U}}_{1}(\boldsymbol{\sigma})=\sum_{s_{1}, s_{2} \in[n]} \overleftarrow{\sigma_{1}}\left(s_{1}\right) \overrightarrow{\sigma_{2}}\left(s_{2}\right) \cdot \mathrm{R}\left[s_{1}, s_{2}\right]+\sum_{s_{1}, s_{2} \in[n]} \overrightarrow{\sigma_{1}}\left(s_{1}\right) \overleftarrow{\sigma_{2}}\left(s_{2}\right) \cdot \mathrm{C}\left[s_{2}, s_{1}\right]$

(Eq. 2) $\widetilde{\mathrm{U}}_{2}(\boldsymbol{\sigma})=\sum_{s_{1}, s_{2} \in[n]} \overleftarrow{\sigma_{1}}\left(s_{1}\right) \overrightarrow{\sigma_{2}}\left(s_{2}\right) \cdot \mathrm{C}\left[s_{1}, s_{2}\right]+\sum_{s_{1}, s_{2} \in[n]} \overrightarrow{\sigma_{1}}\left(s_{1}\right) \overleftarrow{\sigma_{2}}\left(s_{2}\right) \cdot \mathrm{R}\left[s_{2}, s_{1}\right]$

Proof: It is evident from Figure 3 that

$$
\begin{aligned}
\widetilde{\mathrm{U}}_{1}(\boldsymbol{\sigma}) & =\mathbb{E}_{\left(s_{1}, s_{2}\right) \sim \sigma} \mathrm{S}\left[s_{1}, s_{2}\right] \\
& =\mathbb{E}_{\left(s_{1}, s_{2}\right) \sim\left(\overleftarrow{\sigma_{1}}, \overrightarrow{\sigma_{2}}\right)} \mathrm{R}\left[s_{1}, s_{2}\right]+\mathbb{E}_{\left(s_{1}, s_{2}\right) \sim\left(\overrightarrow{\sigma_{1}}, \overleftarrow{\sigma_{2}}\right)} \mathrm{C}^{\mathrm{T}}\left[s_{1}, s_{2}\right] \\
& =\mathbb{E}_{\left(s_{1}, s_{2}\right) \sim\left(\overleftarrow{\sigma_{1}}, \overrightarrow{\sigma_{2}}\right)} \mathrm{R}\left[s_{1}, s_{2}\right]+\mathbb{E}_{\left(s_{1}, s_{2}\right) \sim\left(\overrightarrow{\sigma_{1}}, \overleftarrow{\sigma_{2}}\right)} \mathrm{C}\left[s_{2}, s_{1}\right] \\
& =\sum_{s_{1}, s_{2} \in[n]} \overleftarrow{\sigma_{1}}\left(s_{1}\right) \overrightarrow{\sigma_{2}}\left(s_{2}\right) \cdot \mathrm{R}\left[s_{1}, s_{2}\right]+\sum_{s_{1}, s_{2} \in[n]} \overrightarrow{\sigma_{1}}\left(s_{1}\right) \overleftarrow{\sigma_{2}}\left(s_{2}\right) \cdot \mathrm{C}\left[s_{2}, s_{1}\right]
\end{aligned}
$$

and

$$
\begin{aligned}
\widetilde{\mathrm{U}}_{2}(\boldsymbol{\sigma}) & =\mathbb{E}_{\left(s_{1}, s_{2}\right) \sim \sigma} \mathrm{S}^{\mathrm{T}}\left[s_{1}, s_{2}\right] \\
& =\mathbb{E}_{\left(s_{1}, s_{2}\right) \sim\left(\overleftarrow{\sigma_{1}}, \overrightarrow{\sigma_{2}}\right)} \mathrm{C}\left[s_{1}, s_{2}\right]+\mathbb{E}_{\left(s_{1}, s_{2}\right) \sim\left(\overrightarrow{\sigma_{1}}, \overleftarrow{\sigma_{2}}\right)} \mathrm{R}^{\mathrm{T}}\left[s_{1}, s_{2}\right] \\
& =\mathbb{E}_{\left(s_{1}, s_{2}\right) \sim\left(\overleftarrow{\sigma_{1}}, \overrightarrow{\sigma_{2}}\right)} \mathrm{C}\left[s_{1}, s_{2}\right]+\mathbb{E}_{\left(s_{1}, s_{2}\right) \sim\left(\overrightarrow{\sigma_{1}}, \overleftarrow{\sigma_{2}}\right)} \mathrm{R}\left[s_{2}, s_{1}\right] \\
& =\sum_{s_{1}, s_{2} \in[n]} \overleftarrow{\sigma_{1}}\left(s_{1}\right) \overrightarrow{\sigma_{2}}\left(s_{2}\right) \cdot \mathrm{C}\left[s_{1}, s_{2}\right]+\sum_{s_{1}, s_{2} \in[n]} \overrightarrow{\sigma_{1}}\left(s_{1}\right) \overleftarrow{\sigma_{2}}\left(s_{2}\right) \cdot \mathrm{R}\left[s_{2}, s_{1}\right]
\end{aligned}
$$


as needed.

By Observation 6.1, it immediately follows:

Observation 6.2 For a given strategy $s \in[2 n]$, the conditional expected utilities are:

$$
\begin{aligned}
& \text { (Eq. 1) } \widetilde{\mathrm{U}}_{1}\left(\boldsymbol{\sigma}_{-1} \diamond s\right)=\left\{\begin{array}{ll}
\sum_{s_{2} \in[n]} \overrightarrow{\sigma_{2}}\left(s_{2}\right) \cdot \mathrm{R}\left[s, s_{2}\right], & s \in[n] \\
\sum_{s_{2} \in[n]} \overleftarrow{\sigma_{2}}\left(s_{2}\right) \cdot \mathrm{C}\left[s_{2}, s-n\right], & s \in[2 n] \backslash[n]
\end{array},\right. \\
& \text { (Eq. 2) } \widetilde{\mathrm{U}}_{2}\left(\boldsymbol{\sigma}_{-2} \diamond s\right)=\left\{\begin{array}{ll}
\sum_{s_{1} \in[n]} \overrightarrow{\sigma_{1}}\left(s_{1}\right) \cdot \mathrm{R}\left[s, s_{1}\right], & s \in[n] \\
\sum_{s_{1} \in[n]} \overleftarrow{\sigma_{1}}\left(s_{1}\right) \cdot \mathrm{C}\left[s_{1}, s-n\right], & s \in[2 n] \backslash[n]
\end{array} .\right.
\end{aligned}
$$

We now prove a characterization of the supports of a Nash equilibrium for the win-lose GHRsymmetrization of a bimatrix game with the positive utility property.

Lemma 6.2 Fix a win-lose bimatrix game $\mathrm{G}=\langle\mathrm{R}, \mathrm{C}\rangle$ with the positive utility property, and consider its win-lose GHR-symmetrization $\widetilde{G}$ with a Nash equilibrium $\boldsymbol{\sigma}$. Then, there are only three possible cases:

(C.1) $\overleftarrow{\sigma_{1}}, \overrightarrow{\sigma_{1}}, \overleftarrow{\sigma_{2}}, \overrightarrow{\sigma_{2}} \neq 0^{n}$

(C.2) $\overleftarrow{\sigma_{1}}, \overrightarrow{\sigma_{2}}=0^{n}$ and $\overrightarrow{\sigma_{1}}, \overleftarrow{\sigma_{2}} \neq 0^{n}$

(C.3) $\overrightarrow{\sigma_{1}}, \overleftarrow{\sigma_{2}}=0^{n}$ and $\overleftarrow{\sigma_{1}}, \overrightarrow{\sigma_{2}} \neq 0^{n}$

Proof: Since $\sigma_{1}$ and $\sigma_{2}$ are mixed strategies, the following four implications hold trivially:

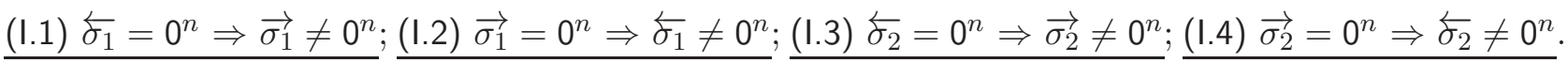

We now prove: (I.5) $\overleftarrow{\sigma_{1}}=0^{n} \Rightarrow \overleftarrow{\sigma_{2}} \neq 0^{n}$ : Assume, by way of contradiction, that $\overleftarrow{\sigma_{1}}=0^{n}$ and $\overleftarrow{\sigma_{2}}=0^{n}$. Then, no profile $\left\langle s_{1}, s_{2}\right\rangle$ is supported in $\left\langle\overleftarrow{\sigma_{1}}, \overrightarrow{\sigma_{2}}\right\rangle$ and no profile $\left\langle s_{1}, s_{2}\right\rangle$ is supported in $\left\langle\overrightarrow{\sigma_{1}}, \overleftarrow{\sigma_{2}}\right\rangle$. By the formula for $\widetilde{U}_{1}(\boldsymbol{\sigma})$ in Observation 6.1 (Eq. 1), these imply that $\widetilde{\mathrm{U}}_{1}(\boldsymbol{\sigma})=0$. By Lemmas 2.2 and 6.1, $\widetilde{\mathrm{U}}_{1}(\boldsymbol{\sigma})>0$. A contradiction. In a corresponding way,

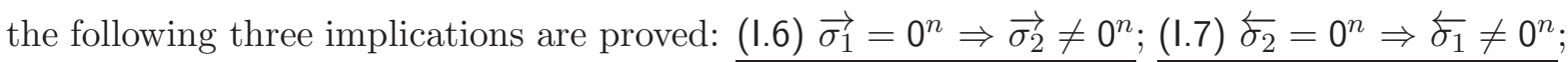
(I.8) $\overrightarrow{\sigma_{2}}=0^{n} \Rightarrow \overrightarrow{\sigma_{1}} \neq 0^{n}$.

We finally prove: (I.9) $\overleftarrow{\sigma_{1}}=0^{n} \Rightarrow \overrightarrow{\sigma_{2}}=0^{n}$. Assume, by way of contradiction, that $\overleftarrow{\sigma_{1}}=0^{n}$ and $\overrightarrow{\sigma_{2}} \neq 0^{n}$. Then, there is a strategy $s_{2} \in[2 n] \backslash[n]$ with $\overrightarrow{\sigma_{2}}\left(s_{2}-n\right)>0 ;$ so, $s_{2} \in \operatorname{Supp}\left(\sigma_{2}\right)$. Since $\overleftarrow{\sigma_{1}}=0^{n}$, it follows from the formula for $\widetilde{\mathrm{U}}_{2}(\boldsymbol{\sigma})$ in Observation 6.2 (Eq. 2) with $\boldsymbol{\sigma}=\boldsymbol{\sigma}_{-2} \diamond s_{2}$ that $\widetilde{\mathrm{U}}_{2}\left(\boldsymbol{\sigma}_{-2} \diamond s_{2}\right)=0$. Since $\boldsymbol{\sigma}$ is a Nash equilibrium and $s_{2} \in \operatorname{Supp}\left(\sigma_{2}\right)$, Lemma 2.1 (Condition (1)) implies that $\widetilde{\mathrm{U}}_{2}(\boldsymbol{\sigma})=0$. By Lemmas 2.2 and 6.1, $\widetilde{\mathrm{U}}_{2}(\boldsymbol{\sigma})>0$. A contradiction. In

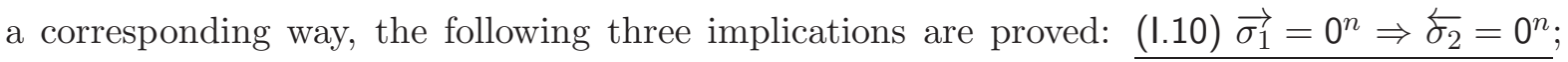

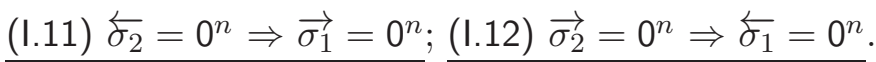

The claim follows now from the twelve implications. 


\subsection{The Balanced Mixture}

Fix a win-lose bimatrix game $\langle\mathrm{R}, \mathrm{C}\rangle$ with the positive utility property. We revisit a composition among either (i) a pair of Nash equilibria for $\langle\mathrm{R}, \mathrm{C}\rangle$ or (ii) a Nash equilibrium for $\langle\mathrm{R}, \mathrm{C}\rangle$ and the tuple of null vectors $\left\langle 0^{n}, 0^{n}\right\rangle$ from [22].

Definition 6.2 Given $\boldsymbol{\rho}=\left\langle\rho_{1}, \rho_{2}\right\rangle \in \mathcal{N} \mathcal{E}(\langle\mathrm{R}, \mathrm{C}\rangle) \cup\left\{\left\langle 0^{n}, 0^{n}\right\rangle\right\}$ and $\boldsymbol{\tau}=\left\langle\tau_{1}, \tau_{2}\right\rangle \in$ $\mathcal{N E}(\langle\mathrm{R}, \mathrm{C}\rangle)$, the balanced mixture of $\boldsymbol{\rho}$ and $\boldsymbol{\tau}$ is the pair

$$
\boldsymbol{\rho} * \boldsymbol{\tau}:=\left\langle\frac{\overline{\mathrm{U}}_{1}(\boldsymbol{\tau})}{\overline{\mathrm{U}}_{1}(\boldsymbol{\tau})+\overline{\mathrm{U}}_{2}(\boldsymbol{\rho})} \rho_{1} \circ \frac{\overline{\mathrm{U}}_{2}(\boldsymbol{\rho})}{\overline{\mathrm{U}}_{1}(\boldsymbol{\tau})+\overline{\mathrm{U}}_{2}(\boldsymbol{\rho})} \tau_{2}, \frac{\overline{\mathrm{U}}_{1}(\boldsymbol{\rho})}{\overline{\mathrm{U}}_{1}(\boldsymbol{\rho})+\overline{\mathrm{U}}_{2}(\boldsymbol{\tau})} \tau_{1} \circ \frac{\overline{\mathrm{U}}_{2}(\boldsymbol{\tau})}{\overline{\mathrm{U}}_{1}(\boldsymbol{\rho})+\overline{\mathrm{U}}_{2}(\boldsymbol{\tau})} \rho_{2}\right\rangle,
$$

and

$$
\boldsymbol{\tau} * \boldsymbol{\rho}:=\left\langle\frac{\overline{\mathrm{U}}_{1}(\boldsymbol{\rho})}{\overline{\mathrm{U}}_{1}(\boldsymbol{\rho})+\overline{\mathrm{U}}_{2}(\boldsymbol{\tau})} \tau_{1} \circ \frac{\overline{\mathrm{U}}_{2}(\boldsymbol{\tau})}{\overline{\mathrm{U}}_{1}(\boldsymbol{\rho})+\overline{\mathrm{U}}_{2}(\boldsymbol{\tau})} \rho_{2}, \frac{\overline{\mathrm{U}}_{1}(\boldsymbol{\tau})}{\overline{\mathrm{U}}_{1}(\boldsymbol{\tau})+\overline{\mathrm{U}}_{2}(\boldsymbol{\rho})} \rho_{1} \circ \frac{\overline{\mathrm{U}}_{2}(\boldsymbol{\rho})}{\overline{\mathrm{U}}_{1}(\boldsymbol{\tau})+\overline{\mathrm{U}}_{2}(\boldsymbol{\rho})} \tau_{2}\right\rangle .
$$

Roughly speaking, the balanced mixture doubles the strategy set of each player by concatenating it to itself; it uses a suitable combination of the probabilities in $\rho$ and $\tau$ and concatenates together the resulting mixed strategies without modifying the players' supports in $\rho$ and $\tau$. Since $\langle\mathrm{R}, \mathrm{C}\rangle$ has the positive utility property, Lemma 2.2 implies that both $\overline{\mathrm{U}}_{1}(\boldsymbol{\tau})>0$ and $\overline{\mathrm{U}}_{2}(\boldsymbol{\tau})>0$; since $\langle\mathrm{R}, \mathrm{C}\rangle$ is win-lose, both $\overline{\mathrm{U}}_{1}(\boldsymbol{\rho}) \geq 0$ and $\overline{\mathrm{U}}_{2}(\boldsymbol{\rho}) \geq 0$. It follows that both $\boldsymbol{\rho} * \boldsymbol{\tau}$ and $\boldsymbol{\tau} * \boldsymbol{\rho}$ are well-defined. It is straightforward to see that $\boldsymbol{\rho} * \boldsymbol{\tau}$ and $\boldsymbol{\tau} * \boldsymbol{\rho}$ are mixed profiles for the win-lose GHR-symmetrization. Thus, a balanced mixture maps the pair of $\boldsymbol{\rho}$ and $\boldsymbol{\tau}$ to the pair of mixed profiles $\boldsymbol{\rho} * \boldsymbol{\tau}$ and $\boldsymbol{\tau} * \boldsymbol{\rho}$ for the win-lose GHR-symmetrization. (We shall provide an inverse map in Theorem 6.6.)

Note that the balanced mixture is an injective map as long as the supports (but not the probabilities) are concerned. Note that when $\boldsymbol{\rho}=\left\langle 0^{n}, 0^{n}\right\rangle$, then $\boldsymbol{\rho} * \boldsymbol{\tau}=\left\langle 0^{n} \circ \tau_{2}, \tau_{1} \circ 0^{n}\right\rangle$ and $\boldsymbol{\tau} * \boldsymbol{\rho}=\left\langle\tau_{1} \circ 0^{n}, 0^{n} \circ \tau_{2}\right\rangle$; so, in $\boldsymbol{\rho} * \boldsymbol{\tau}$ and $\boldsymbol{\tau} * \boldsymbol{\rho}$, the mixed strategies $\tau_{1}$ and $\tau_{2}$ are swapped between the two players, while each is concatenated with $0^{n}$ from left and right, respectively. By abuse of notation, we shall often refer to each of $\rho * \tau$ and $\boldsymbol{\tau} * \boldsymbol{\rho}$ as a balanced mixture. Note that $\boldsymbol{\rho} * \boldsymbol{\tau}$ together with $\boldsymbol{\tau} * \boldsymbol{\rho}$ form a symmetric pair of mixed profiles: setting $\boldsymbol{\phi}:=\boldsymbol{\rho} * \boldsymbol{\tau}$ with $\boldsymbol{\phi}=\left\langle\phi_{1}, \phi_{2}\right\rangle$ yields that $\boldsymbol{\tau} * \boldsymbol{\rho}=\left\langle\phi_{2}, \phi_{1}\right\rangle$. Clearly, $\boldsymbol{\rho} * \boldsymbol{\tau}$ is uniform (resp., symmetric) if and only if $\boldsymbol{\tau} * \boldsymbol{\rho}$ is. We observe a simple uniformity property of the balanced mixture:

Observation 6.3 If (1) $\boldsymbol{\rho}=\left\langle 0^{n}, 0^{n}\right\rangle$, or (2) either $\boldsymbol{\rho}$ or $\boldsymbol{\tau}$ is non-uniform, then both $\boldsymbol{\rho} * \boldsymbol{\tau}$ and $\boldsymbol{\tau} * \boldsymbol{\rho}$ are non-uniform; thus, if $\boldsymbol{\rho} * \boldsymbol{\tau}$ and $\boldsymbol{\tau} * \boldsymbol{\rho}$ are uniform, then both $\boldsymbol{\rho}$ and $\boldsymbol{\tau}$ are uniform.

We further prove a simple symmetry property of the balanced mixture: 
Lemma 6.3 A balanced mixture is symmetric if and only if it is the balanced mixture $\boldsymbol{\tau} * \boldsymbol{\tau}$ for some Nash equilibrium $\boldsymbol{\tau} \in \mathcal{N} \mathcal{E}(\langle\mathrm{R}, \mathrm{C}\rangle)$.

Proof: By Definition 6.2, the balanced mixture $\tau * \tau$ is symmetric for $\tau \in \mathcal{N} \mathcal{E}(\langle\mathrm{R}, \mathrm{C}\rangle)$. In the other direction, consider the symmetric balanced mixture $\boldsymbol{\rho} * \boldsymbol{\tau}$, with $\boldsymbol{\rho} \in \mathcal{N} \mathcal{E}(\langle\mathrm{R}, \mathrm{C}\rangle) \cup\left\{\left\langle 0^{n}, 0^{n}\right\rangle\right\}$ and $\tau \in \mathcal{N} \mathcal{E}(\langle\mathrm{R}, \mathrm{C}\rangle)$; we shall prove that $\boldsymbol{\rho}=\boldsymbol{\tau} \in \mathcal{N} \mathcal{E}(\langle\mathrm{R}, \mathrm{C}\rangle)$. Since $\boldsymbol{\rho} * \boldsymbol{\tau}$ is symmetric, we have that

$$
\frac{\overline{\mathrm{U}}_{1}(\boldsymbol{\tau})}{\overline{\mathrm{U}}_{1}(\boldsymbol{\tau})+\overline{\mathrm{U}}_{2}(\boldsymbol{\rho})} \rho_{1}=\frac{\overline{\mathrm{U}}_{1}(\boldsymbol{\rho})}{\overline{\mathrm{U}}_{1}(\boldsymbol{\rho})+\overline{\mathrm{U}}_{2}(\boldsymbol{\tau})} \tau_{1}
$$

and

$$
\frac{\overline{\mathrm{U}}_{2}(\boldsymbol{\rho})}{\overline{\mathrm{U}}_{1}(\boldsymbol{\tau})+\overline{\mathrm{U}}_{2}(\boldsymbol{\rho})} \tau_{2}=\frac{\overline{\mathrm{U}}_{2}(\boldsymbol{\tau})}{\overline{\mathrm{U}}_{1}(\boldsymbol{\rho})+\overline{\mathrm{U}}_{2}(\boldsymbol{\tau})} \rho_{2} .
$$

This implies that $\rho_{1} \neq 0^{n}$ and $\rho_{2} \neq 0^{n}$, so that $\boldsymbol{\rho} \neq\left\langle 0^{n}, 0^{n}\right\rangle$, which implies that $\boldsymbol{\rho} \in \mathcal{N} \mathcal{E}(\langle\mathrm{R}, \mathrm{C}\rangle)$. Since $\rho_{1}, \rho_{2}, \tau_{1}$ and $\tau_{2}$ are mixed strategies with each summing up its probabilities to 1 , it follows that $\rho_{1}=\tau_{1}$ and $\rho_{2}=\tau_{2}$; so, $\boldsymbol{\rho}=\boldsymbol{\tau}$, as needed. An identical argument applies to the balanced mixture $\boldsymbol{\tau} * \boldsymbol{\rho}$.

Finally, we observe:

Lemma 6.4 Consider the pair of Nash equilibria $\boldsymbol{\tau}, \boldsymbol{\rho} \in \mathcal{N} \mathcal{E}(\langle\mathrm{R}, \mathrm{C}\rangle)$. Then, for each player $i \in[2], \widetilde{\mathrm{U}}_{i}(\boldsymbol{\tau} * \boldsymbol{\rho})<\max \left\{\overline{\mathrm{U}}_{1}(\boldsymbol{\rho}), \overline{\mathrm{U}}_{1}(\boldsymbol{\tau}), \overline{\mathrm{U}}_{2}(\boldsymbol{\rho}), \overline{\mathrm{U}}_{2}(\boldsymbol{\tau})\right\}$.

Proof: First set $i:=1$. By Observation 6.1,

$$
\begin{aligned}
\widetilde{\mathrm{U}}_{1}(\boldsymbol{\tau} * \boldsymbol{\rho})= & \sum_{s_{1}, s_{2} \in[n]} \frac{\overline{\mathrm{U}}_{1}(\boldsymbol{\tau})}{\overline{\mathrm{U}}_{1}(\boldsymbol{\tau})+\overline{\mathrm{U}}_{2}(\boldsymbol{\rho})} \rho_{1}\left(s_{1}\right) \cdot \frac{\overline{\mathrm{U}}_{2}(\boldsymbol{\tau})}{\overline{\mathrm{U}}_{1}(\boldsymbol{\rho})+\overline{\mathrm{U}}_{2}(\boldsymbol{\tau})} \rho_{2}\left(s_{2}\right) \cdot \mathrm{R}\left[s_{1}, s_{2}\right] \\
& +\sum_{s_{1}, s_{2} \in[n]} \frac{\overline{\mathrm{U}}_{2}(\boldsymbol{\rho})}{\overline{\mathrm{U}}_{1}(\boldsymbol{\tau})+\overline{\mathrm{U}}_{2}(\boldsymbol{\rho})} \tau_{2}\left(s_{1}\right) \cdot \frac{\overline{\mathrm{U}}_{1}(\boldsymbol{\rho})}{\overline{\mathrm{U}}_{1}(\boldsymbol{\rho})+\overline{\mathrm{U}}_{2}(\boldsymbol{\tau})} \tau_{1}\left(s_{2}\right) \cdot \mathrm{C}\left[s_{2}, s_{1}\right]
\end{aligned}
$$

Note that $\frac{\overline{\mathrm{U}}_{1}(\boldsymbol{\tau})}{\overline{\mathrm{U}}_{1}(\boldsymbol{\tau})+\overline{\mathrm{U}}_{2}(\boldsymbol{\rho})}, \frac{\overline{\mathrm{U}}_{2}(\boldsymbol{\tau})}{\overline{\mathrm{U}}_{1}(\boldsymbol{\rho})+\overline{\mathrm{U}}_{2}(\boldsymbol{\tau})}, \frac{\overline{\mathrm{U}}_{2}(\boldsymbol{\rho})}{\overline{\mathrm{U}}_{1}(\boldsymbol{\tau})+\overline{\mathrm{U}}_{2}(\boldsymbol{\rho})}, \frac{\overline{\mathrm{U}}_{1}(\boldsymbol{\rho})}{\overline{\mathrm{U}}_{1}(\boldsymbol{\rho})+\overline{\mathrm{U}}_{2}(\boldsymbol{\tau})}<1$, with

$$
\frac{\overline{\mathrm{U}}_{1}(\boldsymbol{\sigma})}{\overline{\mathrm{U}}_{1}(\boldsymbol{\tau})+\overline{\mathrm{U}}_{2}(\boldsymbol{\rho})} \cdot \frac{\overline{\mathrm{U}}_{2}(\boldsymbol{\tau})}{\overline{\mathrm{U}}_{1}(\boldsymbol{\rho})+\overline{\mathrm{U}}_{2}(\boldsymbol{\tau})}+\frac{\overline{\mathrm{U}}_{2}(\boldsymbol{\rho})}{\overline{\mathrm{U}}_{1}(\boldsymbol{\tau})+\overline{\mathrm{U}}_{2}(\boldsymbol{\rho})} \cdot \frac{\overline{\mathrm{U}}_{1}(\boldsymbol{\rho})}{\overline{\mathrm{U}}_{1}(\boldsymbol{\rho})+\overline{\mathrm{U}}_{2}(\boldsymbol{\tau})}<1 .
$$

It follows that

$$
\begin{aligned}
\widetilde{\mathrm{U}}_{1}(\boldsymbol{\tau} * \boldsymbol{\rho}) & <\max \left\{\sum_{s_{1}, s_{2} \in[n]} \rho_{1}\left(s_{1}\right) \cdot \rho_{2}\left(s_{2}\right) \cdot \mathrm{R}\left[s_{1}, s_{2}\right], \sum_{s_{1}, s_{2} \in[n]} \tau_{2}\left(s_{1}\right) \cdot \tau_{1}\left(s_{2}\right) \cdot \mathrm{C}\left[s_{1}, s_{2}\right]\right\} \\
& =\max \left\{\overline{\mathrm{U}}_{1}(\boldsymbol{\rho}), \overline{\mathrm{U}}_{2}(\boldsymbol{\tau})\right\} .
\end{aligned}
$$

In a corresponding way, we establish that $\widetilde{\mathrm{U}}_{2}(\boldsymbol{\tau} * \boldsymbol{\rho})<\max \left\{\overline{\mathrm{U}}_{2}(\boldsymbol{\rho}), \overline{\mathrm{U}}_{1}(\boldsymbol{\tau})\right\}$. From these together, the claim follows. 


\subsection{Characterization of Nash Equilibria}

Loosely speaking, Theorem 6.5] establishes that the balanced mixture yields Nash equilibria for the win-lose GHR-symmetrization, which are thus induced by given Nash equilibria of the bimatrix game $\mathrm{G}=\langle\mathrm{R}, \mathrm{C}\rangle$ with the positive utility property; Theorem 6.6 establishes that every Nash equilibrium for the win-lose GHR-symmetrization is the balanced mixture of either a pair of Nash equilibria for the bimatrix game $\langle\mathrm{R}, \mathrm{C}\rangle$ or a Nash equilibrium with the null vector. We first prove that the balanced mixture maps to the set of Nash equilibria for $\widetilde{G}$.

Theorem 6.5 Consider the bimatrix game $\mathrm{G}=\langle\mathrm{R}, \mathrm{C}\rangle$ with the positive utility property and its win-lose GHR-symmetrization $\widetilde{\mathrm{G}}$. Fix $\boldsymbol{\rho} \in \mathcal{N} \mathcal{E}(\langle\mathrm{R}, \mathrm{C}\rangle) \cup\left\{\left\langle 0^{n}, 0^{n}\right\rangle\right\}$ and $\boldsymbol{\tau} \in \mathcal{N} \mathcal{E}(\langle\mathrm{R}, \mathrm{C}\rangle)$. Then, $\boldsymbol{\rho} * \boldsymbol{\tau}, \boldsymbol{\tau} * \boldsymbol{\rho} \in \mathcal{N} \mathcal{E}(\widetilde{\mathrm{G}})$.

Proof: We prove that $\boldsymbol{\rho} * \boldsymbol{\tau} \in \mathcal{N} \mathcal{E}(\widetilde{\mathrm{G}})$. Denote $\boldsymbol{\phi}=\left\langle\phi_{1}, \phi_{2}\right\rangle:=\boldsymbol{\rho} * \boldsymbol{\tau}$. Consider a strategy $j \in[n]$ with $j \in \operatorname{Supp}\left(\phi_{1}\right)$. By the definition of the balanced mixture, this implies that $j \in \operatorname{Supp}\left(\rho_{1}\right)$. It follows that $\rho \in \mathcal{N} \mathcal{E}(\langle\mathrm{R}, \mathrm{C}\rangle)$. Hence,

$$
\begin{aligned}
& \overline{\mathrm{U}}_{1}(\rho) \\
& =\quad \overline{\mathrm{U}}_{1}\left(\boldsymbol{\rho}_{-1} \diamond j\right) \quad \text { (by Lemma 2.1 }(\text { Condition (1))) } \\
& =\quad \sum_{s_{2} \in[n]} \rho_{2}\left(s_{2}\right) \cdot \mathrm{R}\left[j, s_{2}\right] \quad \text { (from the definition of }\langle\mathrm{R}, \mathrm{C}\rangle \text { ) } \\
& =\max _{\ell \in[n]}\left\{\sum_{s_{2} \in[n]} \rho_{2}\left(s_{2}\right) \cdot \mathrm{R}\left[\ell, s_{2}\right]\right\} \quad \text { (by Lemma 2.1 (Condition (2))). }
\end{aligned}
$$

Hence, we get

$$
\begin{array}{rlrl} 
& \widetilde{\mathrm{U}}_{1}\left(\boldsymbol{\phi}_{-1} \diamond j\right) & & \text { (by Observation 6.2 (Eq. 1)) } \\
= & \frac{\sum_{s_{2} \in[n]} \overrightarrow{\phi_{2}}\left(s_{2}\right) \cdot \mathrm{R}\left[j, s_{2}\right]}{\overline{\mathrm{U}}_{1}(\boldsymbol{\boldsymbol { \rho }})+\overline{\mathrm{U}}_{2}(\boldsymbol{\tau})} \sum_{s_{2} \in[n]} \rho_{2}\left(s_{2}\right) \cdot \mathrm{R}\left[j, s_{2}\right] & & \text { (from the definition of } \boldsymbol{\phi}) \\
= & \frac{\overline{\mathrm{U}}_{2}(\boldsymbol{\tau})}{\overline{\mathrm{U}}_{1}(\boldsymbol{\rho})+\overline{\mathrm{U}}_{2}(\boldsymbol{\tau})} \underbrace{\max _{\ell \in[n]}\left\{\sum_{s_{2} \in[n]} \rho_{2}\left(s_{2}\right) \cdot \mathrm{R}\left[\ell, s_{2}\right]\right\}}_{=\overline{\mathrm{U}}_{1}(\boldsymbol{\rho})} & \\
= & \max _{\ell \in[n]\left\{\sum_{s_{2} \in[2 n] \backslash[n]} \vec{\phi}_{2}\left(s_{2}\right) \cdot \mathrm{R}\left[\ell, s_{2}\right]\right\}} & \text { (from the definition of } \boldsymbol{\phi}) \\
= & \max _{\ell \in[n]}\left\{\widetilde{\mathrm{U}}_{1}\left(\boldsymbol{\phi}_{-1} \diamond \ell\right)\right\} & \text { (by Observation 6.2 (Eq. 1)). }
\end{array}
$$

Hence, restricted to strategies from $[n]$, strategy $j \in[n]$ is a best-response for player 1 in $\phi$, with

$$
\widetilde{\mathrm{U}}_{1}\left(\phi_{-1} \diamond j\right)=\frac{\overline{\mathrm{U}}_{2}(\boldsymbol{\tau}) \cdot \overline{\mathrm{U}}_{1}(\boldsymbol{\rho})}{\overline{\mathrm{U}}_{1}(\boldsymbol{\rho})+\overline{\mathrm{U}}_{2}(\boldsymbol{\tau})} .
$$


Consider now a strategy $k \in[2 n] \backslash[n]$ with $k \in \operatorname{Supp}\left(\phi_{1}\right)$. By the definition of the balanced mixture, this implies that $k-n \in \operatorname{Supp}\left(\tau_{2}\right)$. It follows that $\boldsymbol{\rho} \in \mathcal{N} \mathcal{E}(\langle\mathrm{R}, \mathrm{C}\rangle)$. Hence,

$$
\begin{array}{llcl} 
& \overline{\mathrm{U}}_{2}(\boldsymbol{\tau}) & & \\
= & \overline{\mathrm{U}}_{1}\left(\boldsymbol{\tau}_{-2} \diamond(k-n)\right) & & \text { (by Lemma 2.1 }(\text { Condition }(1))) \\
= & \sum_{s_{1} \in[n]} \tau_{1}\left(s_{1}\right) \cdot \mathrm{C}\left[s_{1}, k-n\right] & & (\text { from the definition of }\langle\mathrm{R}, \mathrm{C}\rangle) \\
= & \max _{\ell \in[2 n] \backslash[n]}\left\{\sum_{s_{1} \in[n]} \tau_{1}\left(s_{1}\right) \cdot \mathrm{C}\left[s_{1}, \ell-n\right]\right\} & & \text { (by Lemma 2.1] (Condition }(2)))
\end{array}
$$

Hence, we get

$$
\begin{aligned}
& \widetilde{U}_{1}\left(\phi_{-1} \diamond k\right) \\
& =\quad \sum_{s_{1} \in[n]} \overleftarrow{\phi_{2}}\left(s_{1}\right) \cdot \mathrm{C}\left[s_{1}, k-n\right] \quad \text { (by Observation } 6.2 \text { (Eq. 1)) } \\
& =\quad \frac{\overline{\mathrm{U}}_{1}(\boldsymbol{\rho})}{\overline{\mathrm{U}}_{1}(\boldsymbol{\rho})+\overline{\mathrm{U}}_{2}(\boldsymbol{\tau})} \sum_{s_{1} \in[n]} \tau_{1}\left(s_{1}\right) \cdot \mathrm{C}\left[s_{1}, k-n\right] \quad \text { (from the definition of } \boldsymbol{\phi} \text { ) } \\
& =\frac{\overline{\mathrm{U}}_{1}(\boldsymbol{\rho})}{\overline{\mathrm{U}}_{1}(\boldsymbol{\rho})+\overline{\mathrm{U}}_{2}(\boldsymbol{\tau})} \underbrace{\max _{\ell \in[2 n] \backslash[n]}\left\{\sum_{s_{1} \in[n]} \tau_{1}\left(s_{1}\right) \cdot \mathrm{C}\left[s_{1}, \ell-n\right]\right\}}_{=\overline{\mathrm{U}}_{2}(\boldsymbol{\tau})} \\
& =\max _{\ell \in[2 n] \backslash[n]}\left\{\sum_{s_{1} \in[n]} \overleftarrow{\phi_{2}}\left(s_{1}\right) \cdot \mathrm{C}\left[s_{1}, \ell-n\right]\right\} \quad \text { (from the definition of } \boldsymbol{\phi} \text { ) } \\
& =\quad \max _{\ell \in[2 n] \backslash[n]}\left\{\widetilde{\mathrm{U}}_{1}\left(\phi_{-1} \diamond \ell\right)\right\} \quad \text { (by Observation 6.2 (Eq. 1)), . }
\end{aligned}
$$

Hence, restricted to strategies from $[2 n] \backslash[n]$, strategy $k \in[2 n] \backslash[n]$ is a best-response for player 1 in $\phi$, with

$$
\widetilde{\mathrm{U}}_{1}\left(\phi_{-1} \diamond k\right)=\frac{\overline{\mathrm{U}}_{1}(\boldsymbol{\rho}) \cdot \overline{\mathrm{U}}_{2}(\boldsymbol{\tau})}{\overline{\mathrm{U}}_{1}(\boldsymbol{\rho})+\overline{\mathrm{U}}_{2}(\boldsymbol{\tau})} .
$$

By (11) and (2), $\widetilde{\mathrm{U}}_{1}\left(\phi_{-1} \diamond j\right)=\widetilde{\mathrm{U}}_{1}\left(\phi_{-1} \diamond k\right)$, which implies that all strategies played by player 1 in $\phi_{1}$ are best-responses to $\phi_{2}$. Similarly, we prove that all strategies played by player 2 in $\phi_{2}$ are best-responses to $\phi_{1}$. Hence, $\phi=\boldsymbol{\rho} * \boldsymbol{\tau} \in \mathcal{N} \mathcal{E}(\widetilde{\mathrm{G}})$. Since $\widetilde{\mathrm{G}}$ is a symmetric game, and $\boldsymbol{\rho} * \boldsymbol{\tau}$ together with $\boldsymbol{\tau} * \boldsymbol{\rho}$ form a symmetric pair of mixed profiles, it follows that $\boldsymbol{\tau} * \boldsymbol{\rho} \in \mathcal{N} \mathcal{E}(\widetilde{\mathrm{G}})$.

We now prove that a Nash equilibrium for $\widetilde{G}$ is either the balanced mixture of two Nash equilibria for $\langle R, C\rangle$, or the balanced mixture of a Nash equilibrium for $\langle R, C\rangle$ with $\left\langle 0^{n}, 0^{n}\right\rangle$.

Theorem 6.6 Consider a bimatrix game $\mathrm{G}=\langle\mathrm{R}, \mathrm{C}\rangle$ with the positive utility property, and its win-lose GHR-symmetrization $\widetilde{\mathrm{G}}$ with a Nash equilibrium $\phi$. Then, exactly one of the following conditions hold:

$\left(\mathrm{C}^{\prime} .1\right) \overleftarrow{\phi_{1}}, \overrightarrow{\phi_{1}}, \overleftarrow{\phi_{2}}, \overrightarrow{\phi_{2}} \neq 0^{n}$, with $\phi=\left\langle\overleftarrow{\varphi_{1}}, \overrightarrow{\varphi_{2}}\right\rangle *\left\langle\overleftarrow{\varphi_{2}}, \overrightarrow{\varphi_{1}}\right\rangle$ and $\left\langle\overleftarrow{\varphi_{2}}, \overrightarrow{\varphi_{1}}\right\rangle,\left\langle\overleftarrow{\varphi_{1}}, \overrightarrow{\varphi_{2}}\right\rangle \in \mathcal{N} \mathcal{E}(\langle\mathrm{R}, \mathrm{C}\rangle)$

$\left(\mathrm{C}^{\prime} .2\right) \overleftarrow{\phi_{1}}=\overrightarrow{\phi_{2}}=0^{n}$, with $\phi=\left\langle 0^{n}, 0^{n}\right\rangle *\left\langle\overleftarrow{\varphi_{2}}, \overrightarrow{\varphi_{1}}\right\rangle$ and $\left\langle\overleftarrow{\varphi_{2}}, \overrightarrow{\varphi_{1}}\right\rangle \in \mathcal{N} \mathcal{E}(\langle\mathrm{R}, \mathrm{C}\rangle)$ 
$\left(\mathrm{C}^{\prime} .3\right) \overrightarrow{\phi_{1}}=\overleftarrow{\phi_{2}}=0^{n}$, with $\phi=\left\langle 0^{n}, 0^{n}\right\rangle *\left\langle\overleftarrow{\varphi_{1}}, \overrightarrow{\varphi_{2}}\right\rangle$ and $\left\langle\overleftarrow{\varphi_{1}}, \overrightarrow{\varphi_{2}}\right\rangle \in \mathcal{N} \mathcal{E}(\langle\mathrm{R}, \mathrm{C}\rangle)$

Proof: Note that each of the Conditions $\left(C^{\prime} .1\right),\left(C^{\prime} .2\right)$ and $\left(C^{\prime} .3\right)$ refines Condition (C.1), (C.2) and (C.3), respectively, in Lemma 6.2, of which exactly one holds. We first prove that Condition (C.3) in Lemma 6.2 implies Condition (C'.3). So assume $\overleftarrow{\phi_{1}}, \overrightarrow{\phi_{1}}, \overleftarrow{\phi_{2}}, \overrightarrow{\phi_{2}} \neq 0^{n}$. Consider a strategy $j \in \operatorname{Supp}\left(\overleftarrow{\phi_{1}}\right) ;$ so, $j \in[n]$. By Observation 6.2 (Eq. 1)

$$
\widetilde{\mathrm{U}}_{1}\left(\phi_{-1} \diamond j\right)=\sum_{s_{2} \in[n]} \overrightarrow{\phi_{2}}\left(s_{2}\right) \cdot \mathrm{R}\left[j, s_{2}\right] .
$$

Since $j$ is a best-response for player 1 to $\phi_{2}$, Lemma 2.1 implies that

$$
\sum_{s_{2} \in[n]} \overrightarrow{\phi_{2}}\left(s_{2}\right) \cdot \mathrm{R}\left[j, s_{2}\right]=\max _{\ell \in[n]}\left\{\sum_{s_{2} \in[n]} \overrightarrow{\phi_{2}}\left(s_{2}\right) \cdot \mathrm{R}\left[\ell, s_{2}\right]\right\} .
$$

Clearly, $\overleftarrow{\varphi_{1}}$ is a mixed strategy for player 1 in the game $\langle R, C\rangle$ with $\operatorname{Supp}\left(\overleftarrow{\varphi_{1}}\right)=\operatorname{Supp}\left(\overleftarrow{\phi_{1}}\right)$ Thus, by normalizing $\overrightarrow{\phi_{2}}$, it follows that for each strategy $j \in \operatorname{Supp}\left(\overleftarrow{\varphi_{1}}\right)$,

$$
\sum_{s_{2} \in[n]} \overrightarrow{\varphi_{2}}\left(s_{2}\right) \cdot \mathrm{R}\left[j, s_{2}\right]=\max _{\ell \in[n]}\left\{\sum_{s_{2} \in[n]} \overrightarrow{\varphi_{2}}\left(s_{2}\right) \cdot \mathrm{R}\left[\ell, s_{2}\right]\right\} .
$$

Consider now a strategy $k \in \operatorname{Supp}\left(\overrightarrow{\phi_{2}}\right)$; so, $k \in[n]$. By Observation 6.2 (Eq. 2),

$$
\widetilde{\mathrm{U}}_{2}\left(\phi_{-2} \diamond(k+n)\right)=\sum_{s_{1} \in[n]} \overleftarrow{\phi_{1}}\left(s_{1}\right) \cdot \mathrm{C}\left[s_{1}, k\right]
$$

Since $k$ is a best-response for player 2 to $\phi_{1}$, it follows that

$$
\sum_{s_{1} \in[n]} \overleftarrow{\phi_{1}}\left(s_{1}\right) \cdot \mathrm{C}\left[s_{1}, k\right]=\max _{\ell \in[n]}\left\{\sum_{s_{1} \in[n]} \overleftarrow{\phi_{1}}\left(s_{1}\right) \cdot \mathrm{C}\left[s_{1}, k\right]\right\}
$$

Clearly, $\overrightarrow{\varphi_{2}}$ is a mixed strategy for player 2 in the game $\langle R, C\rangle$ with $\operatorname{Supp}\left(\overrightarrow{\varphi_{2}}\right)=\operatorname{Supp}\left(\overrightarrow{\phi_{2}}\right)$. Thus, by normalizing $\overleftarrow{\phi_{1}}$, it follows that for each strategy $k \in \operatorname{Supp}\left(\overrightarrow{\phi_{2}}\right)$,

$$
\sum_{s_{1} \in[n]} \overleftarrow{\varphi_{1}}\left(s_{1}\right) \cdot \mathrm{C}\left[s_{1}, k\right]=\max _{\ell \in[n]}\left\{\sum_{s_{1} \in[n]} \overleftarrow{\varphi_{1}}\left(s_{1}\right) \cdot \mathrm{C}\left[s_{1}, k\right]\right\}
$$

By (3) and (44), it follows that $\left\langle\overleftarrow{\varphi_{1}}, \overrightarrow{\varphi_{2}}\right\rangle \in \mathcal{N} \mathcal{E}(\langle\mathrm{R}, \mathrm{C}\rangle)$. Similarly, we prove that $\left\langle\overleftarrow{\varphi_{2}}, \overrightarrow{\varphi_{1}}\right\rangle \in$ $\mathcal{N E}(\langle\mathrm{R}, \mathrm{C}\rangle)$. Now it remains to prove that $\phi=\left\langle\overleftarrow{\varphi_{1}}, \overrightarrow{\varphi_{2}}\right\rangle *\left\langle\overleftarrow{\varphi_{2}}, \overrightarrow{\varphi_{1}}\right\rangle$

Fix now strategies $j, k \in \operatorname{Supp}\left(\phi_{1}\right)$ with $j \in[n]$ and $k \in[2 n] \backslash[n]$. Then, by Observation 6.2 (Eq. 1),

$$
\widetilde{\mathrm{U}}_{1}\left(\phi_{-1} \diamond j\right)=\sum_{s_{2} \in[n]} \overrightarrow{\phi_{2}}\left(s_{2}\right) \cdot \mathrm{R}\left[j, s_{2}\right]
$$


and

$$
\widetilde{\mathrm{U}}_{1}\left(\phi_{-1} \diamond k\right)=\sum_{s_{1} \in[n]} \overleftarrow{\phi_{2}}\left(s_{1}\right) \cdot \mathrm{C}\left[s_{1}, k-n\right]
$$

also, for any fixed strategies $j, k \in \operatorname{Supp}\left(\phi_{2}\right)$ with $j \in[n]$ and $k \in[2 n] \backslash[n]$, by Observation 6.2 (Eq. 2),

$$
\widetilde{\mathrm{U}}_{2}\left(\phi_{-2} \diamond k\right)=\sum_{s_{1} \in[n]} \overleftarrow{\phi_{1}}\left(s_{1}\right) \cdot \mathrm{C}\left[s_{1}, k-n\right]
$$

and

$$
\widetilde{\mathrm{U}}_{2}\left(\phi_{-2} \diamond j\right)=\sum_{s_{2} \in[n]} \overrightarrow{\phi_{1}}\left(s_{2}\right) \cdot \mathrm{R}\left[j, s_{2}\right]
$$

Since $\phi$ is a Nash equilibrium for $\widetilde{G}$, Lemma 2.1 (Condition (1)) implies that $\widetilde{U}_{1}\left(\phi_{-1} \diamond j\right)=$ $\widetilde{\mathrm{U}}_{1}\left(\phi_{-1} \diamond k\right)$ and $\widetilde{\mathrm{U}}_{2}\left(\phi_{-2} \diamond j\right)=\widetilde{\mathrm{U}}_{2}\left(\phi_{-2} \diamond k\right)$. Hence,

$$
\sum_{s_{2} \in[n]} \overrightarrow{\phi_{2}}\left(s_{2}\right) \cdot \mathrm{R}\left[j, s_{2}\right]=\sum_{s_{1} \in[n]} \overleftarrow{\phi_{2}}\left(s_{1}\right) \cdot \mathrm{C}\left[s_{1}, k-n\right]
$$

and

$$
\sum_{s_{1} \in[n]} \overleftarrow{\phi_{1}}\left(s_{1}\right) \cdot \mathrm{C}\left[s_{1}, k-n\right]=\sum_{s_{2} \in[n]} \overrightarrow{\phi_{1}}\left(s_{2}\right) \cdot \mathrm{R}\left[j, s_{2}\right],
$$

respectively. First, note that

$$
\begin{aligned}
& \overline{\mathrm{U}}_{1}\left(\left\langle\overleftarrow{\phi_{1}}, \overrightarrow{\phi_{2}}\right\rangle\right) \\
= & \sum_{s_{1} \in[n]} \overleftarrow{\phi_{1}}\left(s_{1}\right)\left(\sum_{s_{2} \in[n]} \overrightarrow{\phi_{2}}\left(s_{2}\right) \mathrm{R}\left[s_{1}, s_{2}\right]\right) \\
= & \left(\sum_{s_{1} \in[n]} \overleftarrow{\phi_{1}}\left(s_{1}\right)\right) \cdot \underbrace{\left(\sum_{s_{2} \in[n]} \overrightarrow{\phi_{2}}\left(s_{2}\right) \mathrm{R}\left[s_{1}, s_{2}\right]\right)}_{\text {for any strategy } s_{1} \in[n] \text { with } \overleftarrow{\phi_{1}}\left(s_{1}\right)>0} \quad \text { (by Lemma 2.1 (Condition (1))) }
\end{aligned}
$$

and

$$
\begin{aligned}
& \overline{\mathrm{U}}_{2}\left(\left\langle\overleftarrow{\phi_{2}}, \overrightarrow{\phi_{1}}\right\rangle\right) \\
= & \sum_{s_{s_{2} \in[n]} \overrightarrow{\phi_{1}}\left(s_{2}\right)\left(\sum_{s_{1} \in[n]} \overleftarrow{\phi_{2}}\left(s_{1}\right) \mathrm{C}\left[s_{1}, s_{2}\right]\right)} \underbrace{}_{\text {for any strategy } s_{2} \in[n] \text { with } \overrightarrow{\phi_{1}}\left(s_{2}\right)>0} \underbrace{\left(\sum_{s_{1} \in[n]} \overleftarrow{\phi_{2}}\left(s_{1}\right) \mathrm{C}\left[s_{1}, s_{2}\right]\right)} \quad \text { (by Lemma 2.1 (Condition (1))). }
\end{aligned}
$$


By (5), it follows that

$$
\overline{\mathrm{U}}_{1}\left(\left\langle\overleftarrow{\phi_{1}}, \overrightarrow{\phi_{2}}\right\rangle\right) \cdot \sum_{s_{2} \in[n]} \overrightarrow{\phi_{1}}\left(s_{2}\right)=\overline{\mathrm{U}}_{2}\left(\left\langle\overleftarrow{\phi_{2}}, \overrightarrow{\phi_{1}}\right\rangle\right) \cdot \sum_{s_{1} \in[n]} \overleftarrow{\phi_{1}}\left(s_{1}\right)
$$

Second, note that

$$
\begin{aligned}
& \overline{\mathrm{U}}_{1}\left(\left\langle\overleftarrow{\phi_{2}}, \overrightarrow{\phi_{1}}\right\rangle\right) \\
= & \sum_{s_{s_{1} \in[n]} \overleftarrow{\phi_{2}}\left(s_{1}\right)\left(\sum_{s_{2} \in[n]} \overrightarrow{\phi_{1}}\left(s_{2}\right) \mathrm{R}\left[s_{1}, s_{2}\right]\right)} \underbrace{\left(\sum_{s_{2} \in[n]} \overrightarrow{\phi_{1}}\left(s_{2}\right) \mathrm{R}\left[s_{1}, s_{2}\right]\right)}_{\text {for any strategy } s_{1} \in[n] \text { with } \overleftarrow{\phi_{2}}\left(s_{1}\right) \overrightarrow{\phi_{1}}\left(s_{2}\right) \mathrm{R}\left[s_{1}, s_{2}\right]} \quad \text { (by Lemma } 2.1 \text { (Condition }(1)))
\end{aligned}
$$

and

$$
\begin{aligned}
& \overline{\mathrm{U}}_{2}\left(\left\langle\overleftarrow{\phi_{1}}, \overrightarrow{\phi_{2}}\right\rangle\right) \\
& =\quad \sum_{s_{1}, s_{2} \in[n]} \overleftarrow{\phi_{1}}\left(s_{1}\right) \overrightarrow{\phi_{2}}\left(s_{2}\right) \mathrm{C}\left[s_{1}, s_{2}\right] \\
& =\quad \sum_{s_{2} \in[n]} \overrightarrow{\phi_{2}}\left(s_{2}\right)\left(\sum_{s_{1} \in[n]} \overleftarrow{\phi_{1}}\left(s_{1}\right) \mathrm{C}\left[s_{1}, s_{2}\right]\right)
\end{aligned}
$$

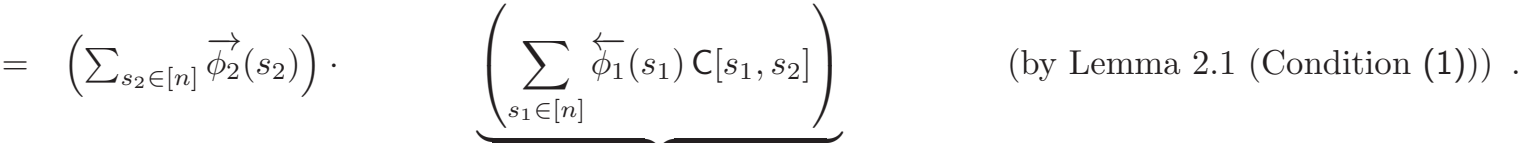

$$
\begin{aligned}
& \text { for any strategy } s_{2} \in[n] \text { with } \overrightarrow{\phi_{2}}\left(s_{2}\right)>0
\end{aligned}
$$

By (6), it follows that

$$
\overline{\mathrm{U}}_{1}\left(\left\langle\overleftarrow{\phi_{2}}, \overrightarrow{\phi_{1}}\right\rangle\right) \cdot \sum_{s_{2} \in[n]} \overrightarrow{\phi_{2}}\left(s_{2}\right)=\overline{\mathrm{U}}_{2}\left(\left\langle\overleftarrow{\phi_{1}}, \overrightarrow{\phi_{2}}\right\rangle\right) \cdot \sum_{s_{1} \in[n]} \overleftarrow{\phi_{2}}\left(s_{1}\right)
$$

Set $\overleftarrow{p_{1}}:=\sum_{s_{1} \in[n]} \overleftarrow{\phi_{1}}\left(s_{1}\right) ; \overrightarrow{p_{1}}, \overleftarrow{p_{2}}$ and $\overrightarrow{p_{2}}$ are defined in a corresponding way. Note that for each $i \in[2], \overleftarrow{p_{i}}+\overrightarrow{p_{i}}=1$. By the definition of the balanced mixture, the first component of the first entry of $\left\langle\overleftarrow{\varphi_{1}}, \overrightarrow{\varphi_{2}}\right\rangle *\left\langle\overleftarrow{\varphi_{2}}, \overrightarrow{\varphi_{1}}\right\rangle$ is

$$
\begin{aligned}
& \frac{\bar{U}_{1}\left(\left\langle\overleftarrow{\varphi_{2}}, \overrightarrow{\varphi_{1}}\right\rangle\right)}{\overline{\mathrm{U}}_{1}\left(\left\langle\overleftarrow{\varphi_{2}}, \overrightarrow{\varphi_{1}}\right\rangle\right)+\overline{\mathrm{U}}_{2}\left(\left\langle\overleftarrow{\varphi_{1}}, \overrightarrow{\varphi_{2}}\right\rangle\right)} \cdot \overleftarrow{\varphi_{1}} \\
& =\frac{\bar{U}_{1}\left(\left\langle\overleftarrow{\phi_{2}}, \overrightarrow{\phi_{1}}\right\rangle\right)}{\overleftarrow{p_{2}} \cdot \overrightarrow{p_{1}} \cdot\left(\frac{\overline{\mathrm{U}}_{1}\left(\left\langle\overleftarrow{\phi_{2}}, \overrightarrow{\phi_{1}}\right\rangle\right)}{\overleftarrow{p_{2}} \cdot \overrightarrow{p_{1}}}+\frac{\overline{\mathrm{U}}_{2}\left(\left\langle\overleftarrow{\phi_{1}}, \overrightarrow{\phi_{2}}\right\rangle\right)}{\overleftarrow{p_{1}} \cdot \overrightarrow{p_{2}}}\right)} \frac{1}{\stackrel{p_{1}}{\overrightarrow{p_{1}}}} \cdot \overleftarrow{\phi_{1}} \\
& =\frac{\overline{\mathrm{U}}_{1}\left(\left\langle\overleftarrow{\phi_{2}}, \overrightarrow{\phi_{1}}\right\rangle\right)}{\overleftarrow{p_{1}} \cdot \overline{\mathrm{U}}_{1}\left(\left\langle\overleftarrow{\phi_{2}}, \overrightarrow{\phi_{1}}\right\rangle\right)+\frac{\overleftarrow{p_{2}} \cdot \overrightarrow{p_{1}}}{\overrightarrow{p_{2}}} \cdot \overline{\mathrm{U}}_{2}\left(\left\langle\overleftarrow{\phi_{1}}, \overrightarrow{\phi_{2}}\right\rangle\right)} \cdot \overleftarrow{\phi_{1}}
\end{aligned}
$$

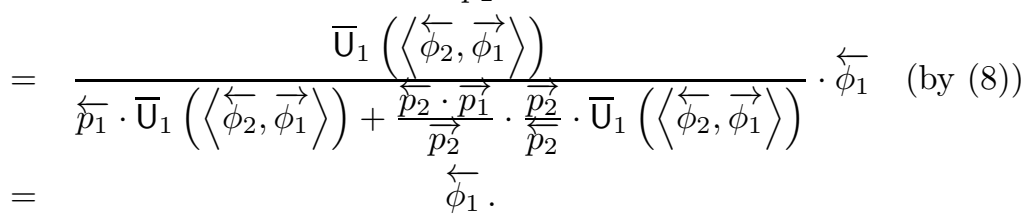


The second component of the first entry of $\left\langle\overleftarrow{\varphi_{1}}, \overrightarrow{\varphi_{2}}\right\rangle *\left\langle\overleftarrow{\varphi_{2}}, \overrightarrow{\varphi_{1}}\right\rangle$ is

$$
\begin{aligned}
\frac{\bar{U}_{2}\left(\left\langle\overleftarrow{\varphi_{1}}, \overrightarrow{\varphi_{2}}\right\rangle\right)}{\overline{\mathrm{U}}_{1}\left(\left\langle\overleftarrow{\varphi_{2}}, \overrightarrow{\varphi_{1}}\right\rangle\right)+\overline{\mathrm{U}}_{2}\left(\left\langle\overleftarrow{\varphi_{1}}, \overrightarrow{\varphi_{2}}\right\rangle\right)} \cdot \overrightarrow{\varphi_{1}} & =\left(1-\frac{{\overline{U_{1}}}_{1}\left(\left\langle\overleftarrow{\varphi_{2}}, \overrightarrow{\varphi_{1}}\right\rangle\right)}{\overline{\mathrm{U}}_{1}\left(\left\langle\overleftarrow{\varphi_{2}}, \overrightarrow{\varphi_{1}}\right\rangle\right)+\overline{\mathrm{U}}_{2}\left(\left\langle\overleftarrow{\varphi_{1}}, \overrightarrow{\varphi_{2}}\right\rangle\right)}\right) \overrightarrow{\varphi_{1}} \\
& =\left(1-\overleftarrow{p_{1}}\right) \cdot \frac{1}{\overrightarrow{p_{1}}} \cdot \overrightarrow{\phi_{1}} \\
& =\overrightarrow{\phi_{1}} .
\end{aligned}
$$

The first component of the second entry of $\left\langle\overleftarrow{\varphi_{1}}, \overrightarrow{\varphi_{2}}\right\rangle *\left\langle\overleftarrow{\varphi_{2}}, \overrightarrow{\varphi_{1}}\right\rangle$ is

$$
\begin{aligned}
& \frac{\bar{U}_{1}\left(\left\langle\overleftarrow{\varphi_{1}}, \overrightarrow{\varphi_{2}}\right\rangle\right)}{\bar{U}_{1}\left(\left\langle\overleftarrow{\varphi_{1}}, \overrightarrow{\varphi_{2}}\right\rangle\right)+\bar{U}_{2}\left(\left\langle\overleftarrow{\varphi_{2}}, \overrightarrow{\varphi_{1}}\right\rangle\right)} \cdot \overleftarrow{\varphi_{2}} \\
& =\frac{\overline{\mathrm{U}}_{1}\left(\left\langle\overleftarrow{\phi_{1}}, \overrightarrow{\phi_{2}}\right\rangle\right)}{\overleftarrow{p_{1}} \cdot \overrightarrow{p_{2}} \cdot\left(\frac{\overline{\mathrm{U}}_{1}\left(\left\langle\overleftarrow{\phi_{1}}, \overrightarrow{\phi_{2}}\right\rangle\right)}{\overleftarrow{p_{1}} \cdot \overrightarrow{p_{2}}}+\frac{\overline{\mathrm{U}}_{2}\left(\left\langle\overleftarrow{\phi_{2}}, \overrightarrow{\phi_{1}}\right\rangle\right)}{\overleftarrow{p_{2}} \cdot \overrightarrow{p_{1}}}\right)} \frac{1}{\overleftarrow{p_{2}}} \cdot \overleftarrow{\phi_{2}} \\
& =\frac{\overline{\mathrm{U}}_{1}\left(\left\langle\overleftarrow{\phi_{1}}, \overrightarrow{\phi_{2}}\right\rangle\right)}{\overleftarrow{p_{1}} \cdot \overline{\mathrm{U}}_{1}\left(\left\langle\overleftarrow{\phi_{2}}, \overrightarrow{\phi_{1}}\right\rangle\right)+\frac{\overleftarrow{p_{2}} \cdot \overrightarrow{\overrightarrow{p_{1}}}}{\overrightarrow{p_{2}}} \cdot \overline{\mathrm{U}}_{2}\left(\left\langle\overleftarrow{\phi_{2}}, \overrightarrow{\phi_{1}}\right\rangle\right)} \cdot \overleftarrow{\phi_{2}}
\end{aligned}
$$

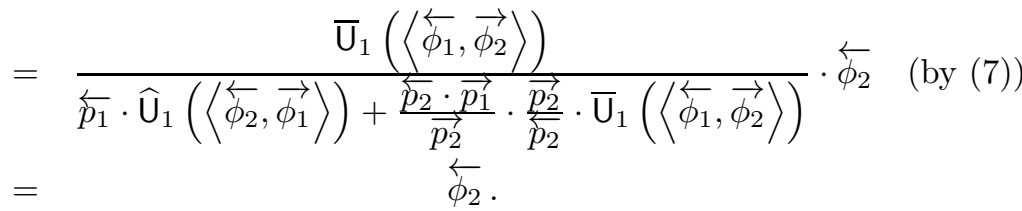

The second component of the second entry of $\left\langle\overleftarrow{\varphi_{1}}, \overrightarrow{\varphi_{2}}\right\rangle *\left\langle\overleftarrow{\varphi_{2}}, \overrightarrow{\varphi_{1}}\right\rangle$ is

$$
\begin{aligned}
\frac{\overline{\mathrm{U}}_{2}\left(\overleftarrow{\varphi_{2}}, \overrightarrow{\varphi_{1}}\right)}{\overline{\mathrm{U}}_{1}\left(\overleftarrow{\varphi_{1}}, \overrightarrow{\varphi_{2}}\right)+\overline{\mathrm{U}}_{2}\left(\overleftarrow{\varphi_{2}}, \overrightarrow{\varphi_{1}}\right)} \cdot \overrightarrow{\varphi_{2}} & =\left(1-\frac{\overline{\mathrm{U}}_{1}\left(\overleftarrow{\varphi_{1}}, \overrightarrow{\varphi_{2}}\right)}{\overline{\mathrm{U}}_{1}\left(\overleftarrow{\varphi_{1}}, \overrightarrow{\varphi_{2}}\right)+\overline{\mathrm{U}}_{2}\left(\overleftarrow{\varphi_{2}}, \overrightarrow{\varphi_{1}}\right)}\right) \overrightarrow{\varphi_{2}} \\
& =\left(1-\overleftarrow{p_{2}}\right) \cdot \frac{1}{\overrightarrow{p_{2}}} \cdot \overrightarrow{\phi_{2}} \\
& =\overrightarrow{\phi_{2}}
\end{aligned}
$$

The proofs that Condition (C.2) (resp., Condition (C.3)) implies Condition (C'.2) (resp., Condition $\left.\left(C^{\prime} .3\right)\right)$ are corresponding.

Recall that the balanced mixture is an injective map as long as the supports (but not the probabilities) are concerned, Hence, Proposition 6.5 implies that

$$
|\mathcal{N E}(\mathrm{GHR}(\mathrm{G}))| \geq|\mathcal{N E} \mathrm{G}|^{2}+2 \cdot|\mathcal{N E}(\mathrm{G})|
$$

(The squared term comes from forming the pair of balanced mixtures $\boldsymbol{\rho} * \boldsymbol{\sigma}$ and $\boldsymbol{\sigma} * \boldsymbol{\rho}$, for each pair $\boldsymbol{\rho}, \boldsymbol{\sigma} \in \mathcal{N} \mathcal{E}\left(\left\langle\mathrm{S}, \mathrm{S}^{\mathrm{T}}\right\rangle\right)$; the linear term comes from forming the pair of balanced mixtures $\boldsymbol{\rho} *\left\langle 0^{n}, 0^{n}\right\rangle$ and $\left\langle 0^{n}, 0^{n}\right\rangle * \boldsymbol{\rho}$, for each $\boldsymbol{\rho} \in \mathcal{N} \mathcal{E}\left(\left\langle\mathrm{S}, \mathrm{S}^{\mathrm{T}}\right\rangle\right)$.) Proposition 6.6 implies that a Nash equilibrium for $\left\langle\mathrm{S}, \mathrm{S}^{\mathrm{T}}\right\rangle$ is induced via the balanced mixture either by a single Nash 
equilibrium (Cases $\left(C^{\prime} .2\right)$ and $\left(C^{\prime} .3\right)$ ) or by a pair of Nash equilibria (Case $\left(C^{\prime} .1\right)$ ) for G. Hence, Proposition 6.6 establishes that the balanced mixture is a surjective map, so that

$$
\begin{aligned}
|\mathcal{N E}(\mathrm{GHR}(\mathrm{G}))| & =|\mathcal{N E}(\mathrm{G})|^{2}+2 \cdot|\mathcal{N} \mathcal{E}(\mathrm{G})| \\
& =|\mathcal{N E}(\mathrm{G})| \cdot(|\mathcal{N E}(\mathrm{G})|+2) .
\end{aligned}
$$

Thus, Theorems 6.5 and 6.6 provide together a complete characterization of the Nash equilibria for the GHR-symmetrization $\left\langle\mathrm{S}, \mathrm{S}^{\mathrm{T}}\right\rangle$ in terms of those for $\langle\mathrm{R}, \mathrm{C}\rangle$; so computing a Nash equilibrium for a win-lose bimatrix game and computing a Nash equilibrium for a symmetric win-lose bimatrix game are polynomially equivalent problems.

\subsection{Complexity of the Search Problem}

Here is an algorithm to compute a Nash equilibrium for a win-lose bimatrix game $G$ with the positive utility property, with a single invocation of an algorithm to compute a Nash equilibrium for a symmetric win-lose bimatrix game with the positive utility property:

(1) Construct the win-lose GHR-symmetrization GHR(G).

(2) Compute a Nash equilibrium $\phi$ for the symmetric win-lose game GHR(G).

(3) Recover a Nash equilibrium for $G$ as follows:

(3.1) If $\overleftarrow{\phi_{1}}, \overrightarrow{\phi_{1}}, \overleftarrow{\phi_{2}}, \overrightarrow{\phi_{2}} \neq 0^{n}$, then output $\left\langle\overleftarrow{\varphi_{2}}, \overrightarrow{\varphi_{1}}\right\rangle\left(\right.$ or $\left.\left\langle\overleftarrow{\varphi_{1}}, \overrightarrow{\varphi_{2}}\right\rangle\right)$

(3.2) If $\overleftarrow{\phi_{1}}=\overrightarrow{\phi_{2}}=0^{n}$, then output $\left\langle\overleftarrow{\varphi_{2}}, \overrightarrow{\varphi_{1}}\right\rangle$

(3.3) If $\overrightarrow{\phi_{1}}=\overleftarrow{\phi_{2}}=0^{n}$, then output $\left\langle\overleftarrow{\varphi_{1}}, \overrightarrow{\varphi_{2}}\right\rangle$

Correctness follows from Theorem 6.6. Since computing a Nash equilirium for a win-lose bimatrix game with the positive utility property is $\mathcal{P} \mathcal{P} \mathcal{A D}$-hard (Section 3 ), it immediately follows:

Theorem 6.7 Computing a Nash equilirium for a symmetric win-lose bimatrix game with the positive utility property is $\mathcal{P} \mathcal{P} \mathcal{A D}$-complete.

\subsection{Complexity of the Counting Problem}

It was a consequence of Propositions 5.13 and 5.14 that computing the number of Nash equilibria for a win-lose bimatrix game is $\# \mathcal{P}$-hard. We now extend this result to symmetric win-lose bimatrix games. We show:

Theorem 6.8 Computing the number (resp., the parity of the number) of Nash equilibria for a symmetric win-lose bimatrix game is \#P्P-complete (resp., $\oplus \mathcal{P}$-complete). 
Proof: Fix a win-lose gadget game $\widehat{G}$ and a 3SAT formula $\phi$, inducing the win-lose bimatrix game $\mathrm{G}=\mathrm{G}(\widehat{\mathrm{G}}, \phi)$ and the symmetric win-lose bimatrix game $\widetilde{\mathrm{G}}=\mathrm{GHR}(\mathrm{G})$. By Lemma 5.3 (Condition (1)) and Propositions 5.13 and 5.14,

$$
|\mathcal{N E}(\mathrm{G}(\widehat{\mathrm{G}}, \phi))|=|\mathcal{N E}(\widehat{\mathrm{G}})|+\# \phi .
$$

Hence, by Theorems 6.5 and 6.6 .

$$
\begin{aligned}
& |\mathcal{N} \mathcal{E}(\widetilde{\mathrm{G}})| \\
& =\underbrace{(|\mathcal{N E}(\widehat{\mathrm{G}})|+\# \phi)^{2}} \quad+\quad \underbrace{2 \cdot(|\mathcal{N} \mathcal{E}(\widehat{\mathrm{G}})|+\# \phi)} \\
& \text { balanced mixtures of two Nash equilibria balanced mixtures of a Nash equilibrium and }\left\langle 0^{n}, 0^{n}\right\rangle \\
& =(|\mathcal{N} \mathcal{E}(\widehat{\mathrm{G}})|+\# \phi) \cdot(|\mathcal{N} \mathcal{E}(\widehat{\mathrm{G}})|+\# \phi+2) \text {, }
\end{aligned}
$$

which can be solved for $\# \phi$. Since computing $\# \phi$ is $\# \mathcal{P}$-hard [35], the $\# \mathcal{P}$-hardness follows. Note that $\oplus|\mathcal{N} \mathcal{E}(\widetilde{\mathrm{G}})|=\oplus(|\mathcal{N} \mathcal{E}(\widehat{\mathrm{G}})|+\# \phi)$, from which $\oplus \phi$ can be computed. Since computing $\oplus \phi$ is $\oplus \mathcal{P}$-hard [33], the $\oplus \mathcal{P}$-hardness follows.

\section{Complexity Results}

Symmetric win-lose bimatrix games, win-lose bimatrix games and win-lose three-player games are considered in Sections 7.1, 7.2 and 7.3, respectively. We first recall an informal summary of the proof technique from Section 1.3. The win-lose reduction is used to obtain, given a suitable win-lose gadget game $\widehat{G}$ and a formula $\phi$, a win-lose game $\mathrm{G}=\mathrm{G}(\widehat{\mathrm{G}}, \phi)$; Propositions 5.13 and 5.14 are used to relate the properties of the Nash equilibria for G to the satisfiablity of $\phi$. For the case of symmetric win-lose bimatrix games, the GHR-symmetrization from Section 6 is used to obtain from $G$ the symmetric win-lose game $\widetilde{G}=G H R(G)$; Theorems 6.5 and 6.6 are used to relate the properties of the Nash equilibria for $\widetilde{G}$ to those of the Nash equilibria for G. In turn, this results in relating the properties of the Nash equilibria for $\widetilde{G}$ to the satisfiability of $\phi$.

Recall that for bimatrix games, the win-lose reduction sets that the strategy set of each player $i \in[2]$ in $\mathrm{G}$ is $\Sigma_{i}(\mathrm{G})=\widehat{\Sigma}_{i} \cup \mathrm{L} \cup \vee \cup \mathcal{C}$.

\subsection{Symmetric Win-Lose Bimatrix Games}

Recall that the win-lose GHR-symmetrization mirrors the strategies of each player in a bimatrix game. So, for each player $i \in[2], \Sigma_{i}(\widetilde{\mathrm{G}})=\widehat{\Sigma}_{i} \cup \mathrm{L} \cup \vee \cup \mathcal{C} \cup \widehat{\Sigma}_{i}^{\prime} \cup \mathrm{L}^{\prime} \cup \mathrm{V}^{\prime} \cup \mathcal{C}^{\prime}$, where the strategy sets $\widehat{\Sigma}_{i}^{\prime}, \mathrm{L}^{\prime}, \mathrm{V}^{\prime}$ and $\mathcal{C}^{\prime}$ are mirrors of the strategy sets $\widehat{\Sigma}_{i}, \mathrm{~L}, \mathrm{~V}$ and $\mathcal{C}$, respectively. We show:

Theorem 7.1 Restricted to symmetric win-lose bimatrix games, the following decision problems are $\mathcal{N} \mathcal{P}$-complete: 


\begin{tabular}{|l|l|}
\hline \hline Group I & Group III \\
\hline$\exists$ NASH WITH SMALL UTILITIES & $\exists$ NASH WITH LARGE UTILITIES \\
$\exists$ NASH WITH SMALL TOTAL UTILITY & $\exists$ NASH WITH LARGE TOTAL UTILITY \\
$\exists$ NASH WITH LARGE SUPPORTS & $\exists$ NASH WITH SMALL SUPPORTS \\
\cline { 2 - 2 }$\exists$ NASH WITH RESTRICTING SUPPORTS & Group IV \\
\cline { 2 - 2 }$\exists$ NASH WITH RESTRICTED SUPPORTS & $\exists k+1$ NASH (with $k \geq 1$ ) \\
$\exists$ NASH WITH SMALL PROBABILITIES & $\exists \neg$ PARETO-OPTIMAL NASH \\
$\exists \neg$ UNIFORM NASH & $\exists \neg$ STRONGLY PARETO-OPTIMAL NASH \\
\hline Group II & $\exists \neg$ SYMMETRIC NASH \\
\hline$\exists$ UNIFORM NASH & \\
\hline \hline
\end{tabular}

Furthermore, their counting versions are \#P-complete; so is \# SYMMETRIC NASH. Except for $\oplus \neg$ UNIFORM NASH (whose $\oplus \mathcal{P}$-hardness remains open) and for $\oplus \neg$ SYMMETRIC NASH (which is in $\mathcal{P})$, their parity versions are $\oplus \mathcal{P}$-complete.

For each of the four Groups, we fix a different gadget game $\widehat{G}$. For Group $I, \widehat{G}$ is fixed to the win-lose cyclic game $\widehat{G}_{1}[1]$ (Section 4.1). For Group II, $\widehat{G}$ is fixed to the win-lose non-uniform game $\widehat{G}_{3}$ (Section 4.3 ). For Group III, $\widehat{G}$ is fixed to the win-lose cyclic game $\widehat{G}_{1}[h]$, for any integer $h$ with $h>\frac{n}{2}$ (Section 4.1). For Group $I V$, $\widehat{\mathrm{G}}$ is fixed to the win-lose cyclic game $\widehat{\mathrm{G}}_{1}[2]$ (Section 4.1); we also use the win-lose diagonal game $\widehat{G}_{5}[k]$ as a subgame in the last stage of the reduction. Regarding Step 3 in the three-steps proof plan outlined in Section 1.3, the decision problems in Group I and Group III fall under Case (1); the single decision problem in Group II falls under Case (2); the decision problems in Group IV fall under Case (3).

Notation-wise, we shall use, for the proof of Theorem 17.1, the symbols $\mathrm{P}$ and $\mathrm{Q}$ to denote properties of Nash equilibria for the game $\operatorname{GHR}(\mathrm{G}(\widehat{\mathrm{G}}, \phi))$ in the cases where $\phi$ is unsatisfiable and satisfiable, respectively. Loosely speaking, we shall use, to establish $\mathcal{N} \mathcal{P}$-hardness, a $\mathrm{Q}$ property and a contradictory $\mathrm{P}$ property, which together disentangle satisfiability; that is, the $\mathrm{Q}$ property holds if and only if $\phi$ is satisfiable, and this implies the $\mathcal{N} \mathcal{P}$-hardness of deciding the corresponding property. To establish \#P-hardness, we shall express the number of Nash equilibria for $\operatorname{GHR}(\mathrm{G}(\widehat{\mathrm{G}}, \phi))$ fulfilling the property $\mathrm{Q}$ as a function of \# $\phi$; inverting the formula yields $\# \phi$, and $\# \mathcal{P}$-hardness follows. To establish $\oplus \mathcal{P}$-hardness, we shall express the parity of the number of Nash equilibria for $\operatorname{GHR}(\mathrm{G}(\widehat{\mathrm{G}}, \phi))$ fulfilling the property $Q$ as a function of $\oplus \phi$; inverting the expression yields $\oplus \phi$ and $\oplus \mathcal{P}$-hardness follows. We shall use $U$ and $\widetilde{U}$ to denote (expected) utilities for the games $\mathrm{G}=\mathrm{G}(\widehat{\mathrm{G}}, \phi)$ and $\widetilde{\mathrm{G}}=\mathrm{GHR}(\mathrm{G}(\widehat{\mathrm{G}}, \phi))$, respectively. For a game $\mathrm{G}$, denote as $\kappa(\mathrm{G})$ the maximum number of strategies for each player in the game $\mathrm{G}$.

Proof: Consider a 3SAT formula $\phi$ with $n:=|\operatorname{Var}(\phi)| \geq 5$ and a win-lose gadget game $\widehat{\mathrm{G}}$, and their induced win-lose game $\mathrm{G}=\mathrm{G}(\widehat{\mathrm{G}}, \phi)$ constructed by the win-lose reduction (Section 5 ), and 
the win-lose GHR-symmetrization $\widetilde{\mathrm{G}}:=\mathrm{GHR}(\mathrm{G})$ (Section 6). Clearly, $\kappa(\mathrm{G}(\widehat{\mathrm{G}}, \phi))=\kappa(\widehat{\mathrm{G}})+2 n+$ $|\mathcal{C}(\phi)|+n(n+1)$. Hence, $\mathrm{G}=\mathrm{G}(\widehat{\mathrm{G}}, \phi)$ has size polynomial in the size of $\phi$ if and only if $\widehat{\mathrm{G}}$ has size polynomial in the size of $\phi$. It follows, by the win-lose GHR-symmetrization, that $\widetilde{G}$ has size polynomial in the size of $\phi$ if and only if $\widehat{G}$ has size polynomial in the size of $\phi$.

Assume first that $\phi$ is unsatisfiable. Proposition 5.13 implies that $\mathcal{N} \mathcal{E}(\mathrm{G}(\widehat{\mathrm{G}}, \phi))=\mathcal{N} \mathcal{E}(\widehat{\mathrm{G}})$; call each Nash equilibrium for $\mathrm{G}(\widehat{\mathrm{G}}, \phi)$ coming from $\widehat{\mathrm{G}}$ a gadget equilibrium. So there are $|\mathcal{N} \mathcal{E}(\widehat{\mathrm{G}})|$ gadget equilibria for $\mathrm{G}$. By Theorem 6.5, $\widetilde{G}=\mathrm{GHR}(\mathrm{G})$ has the following Nash equilibria, which are balanced mixtures of the gadget equilibria for $\mathrm{G}$ :

(1) $\boldsymbol{\sigma}^{1}=\widehat{\boldsymbol{\sigma}} * \widehat{\boldsymbol{\tau}}$, for each ordered pair $\langle\widehat{\boldsymbol{\sigma}}, \widehat{\boldsymbol{\tau}}\rangle$ of the gadget equilibria $\widehat{\boldsymbol{\sigma}}$ and $\widehat{\boldsymbol{\tau}}$ for $\mathrm{G}$.

(2) $\boldsymbol{\sigma}^{2}=\widehat{\boldsymbol{\sigma}} *\left\langle 0^{\kappa}, 0^{\kappa}\right\rangle=\left\langle\widehat{\sigma}_{1} \circ 0^{\kappa}, 0^{\kappa} \circ \widehat{\sigma}_{2}\right\rangle$ and $\boldsymbol{\sigma}^{3}=\left\langle 0^{\kappa}, 0^{\kappa}\right\rangle * \widehat{\boldsymbol{\sigma}}=\left\langle 0^{\kappa} \circ \widehat{\sigma}_{2}, \widehat{\sigma}_{1} \circ 0^{\kappa}\right\rangle$, for each gadget equilibrium $\widehat{\boldsymbol{\sigma}}$ for $\mathrm{G}$.

By Theorem 6.6, $\widetilde{G}$ has no other Nash equilibrium. Hence, when $\phi$ is unsatisfiable, there are $|\mathcal{N E}(\widehat{\mathrm{G}})|^{2}+2 \cdot|\mathcal{N} \mathcal{E}(\widehat{\mathrm{G}})|$ Nash equilibria for $\widetilde{\mathrm{G}}$.

Assume now that $\phi$ is satisfiable. Then, in addition to the gadget equilibria, it follows, by Proposition 5.14, that $\mathrm{G}(\widehat{\mathrm{G}}, \phi)$ has, for each satisfying assignment $\gamma$ of $\phi$, a Nash equilibrium $\boldsymbol{\sigma}=\boldsymbol{\sigma}(\gamma)$; call $\boldsymbol{\sigma}$ a literal equilibrium. So there are \# $\phi$ literal equilibria for G. By Theorem 6.5, $\widetilde{\mathrm{G}}=\mathrm{GHR}(\mathrm{G})$ has, in addition to the balanced mixtures of gadget equilibria, the following Nash equilibria, called additional, which are either balanced mixtures of literal equilibria for $\mathrm{G}$ or balanced mixtures of a literal and a gadget equilibrium for G:

(1) $\boldsymbol{\sigma}^{4}=\boldsymbol{\sigma} * \boldsymbol{\tau}$, for each ordered pair $\langle\boldsymbol{\sigma}, \boldsymbol{\tau}\rangle$ of the literal equilibria $\boldsymbol{\sigma}$ and $\boldsymbol{\tau}$ for $\mathrm{G}$.

(2) $\boldsymbol{\sigma}^{5}=\boldsymbol{\sigma} *\left\langle 0^{\kappa}, 0^{\kappa}\right\rangle$ and $\boldsymbol{\sigma}^{6}=\left\langle 0^{\kappa}, 0^{\kappa}\right\rangle * \boldsymbol{\sigma}$, for each literal equilibrium $\boldsymbol{\sigma}$ for G.

(3) $\boldsymbol{\sigma}^{7}=\widehat{\boldsymbol{\sigma}} * \boldsymbol{\sigma}$ and $\boldsymbol{\sigma}^{8}=\boldsymbol{\sigma} * \widehat{\boldsymbol{\sigma}}$, for each pair of a gadget equilibrium $\widehat{\boldsymbol{\sigma}}$ and a literal equilibrium $\sigma$ for $\mathrm{G}$.

Since there are $|\mathcal{N E}(\widehat{G})|$ gadget equilibria and $\# \phi$ literal equilibria, it follows that there are $(\# \phi)^{2}+2 \cdot \# \phi=\# \phi \cdot(\# \phi+2)$ balanced mixtures of literal equilibria and $2 \cdot|\mathcal{N} \mathcal{E}(\widehat{\mathrm{G}})| \cdot \# \phi$ balanced mixtures of a gadget equilibrium and a literal equilibrium, respectively. By Theorem 6.6, $\widetilde{G}$ has no other Nash equilibrium.

We proceed to establish properties of the Nash equilibria for $G$ and $\widetilde{G}$, respectively. Loosely speaking, these shall be properties disentangling the satisfiability of $\phi$; thus, $\mathcal{N} \mathcal{P}$-hardness follows. We split the proof into four parts, one for each Group.

Group I: We start with a particular remark about $\exists \neg$ UNIFORM NASH. Proposition [5.14] ensures that when $\phi$ is satisfiable, the literal Nash equilibria for $\mathrm{G}(\widehat{\mathrm{G}}, \phi)$ dismatch non-uniformity 
as they are uniform no matter how $\widehat{G}$ were chosen. So the win-lose reduction is inadequate on its own to ensure the equivalence of non-uniformity to the satisfiability of $\phi$. Although it might seem that techniques based on the win-lose reduction could not be adequate for showing the $\mathcal{N} \mathcal{P}$ hardness of deciding the existence of a non-uniform Nash equilibrium, we shall establish that this is not the case. In Step 1, choose the gadget game $\widehat{G}$ to have a single uniform Nash equilibrium. For Step 2, Proposition 5.13 ensures that $\mathrm{G}(\widehat{\mathrm{G}}, \phi)$ has no non-uniform Nash equilibrium when $\phi$ is unsatisfiable. Hence, Theorems 6.5 and 6.6 ensure that $\widetilde{G}$ has no non-uniform Nash equilibrium when $\phi$ is unsatisfiable. But when $\phi$ is satisfiable, in Step 3, non-uniform Nash equilibria are created for the win-lose GHR-symmetrization of $\mathrm{G}(\widehat{\mathrm{G}}, \phi)$ as balanced mixtures of the single uniform Nash equilibrium for $\mathrm{G}$ (coming from $\widehat{\mathrm{G}}$ ) with some literal Nash equilibrium for $\mathrm{G}(\widehat{\mathrm{G}}, \phi)$. So the win-lose GHR-symmetrization has a non-uniform Nash equilibrium if and only if $\phi$ is satisfiable, and $\mathcal{N} \mathcal{P}$-hardness follows. We stress that the $\mathcal{N} \mathcal{P}$-hardness of $\exists \neg$ UNIFORM NASH for symmetric win-lose bimatrix games exploits the possibility of creating non-uniform Nash equilibria as balanced mixtures of uniform Nash equilibria. Note that this result is subsuming the $\mathcal{N} \mathcal{P}$-hardness of $\exists \neg$ UNIFORM NASH for the more general class of win-lose bimatrix games, for which the win-lose reduction could accomodate no direct proof. We now continue with the formal proof.

Fix $\widehat{G}:=\widehat{G}_{1}[1]$. Since $\kappa\left(\widehat{G}_{1}[1]\right)=1$, it follows that each of $\mathrm{G}=\mathrm{G}(\widehat{\mathrm{G}}, \phi)$ and $\widetilde{\mathrm{G}}=\mathrm{GHR}(\mathrm{G})$ has size polynomial in the size of $\phi$. By Proposition 4.1, $\widehat{G}_{1}[1]$ has a unique Nash equilibrium $\widehat{\boldsymbol{\sigma}}$. Assume first that $\phi$ is unsatisfiable. Since $\mathcal{N} \mathcal{E}\left(\mathrm{G}\left(\widehat{\mathrm{G}}_{1}[1], \phi\right)\right)=\mathcal{N} \mathcal{E}\left(\widehat{\mathrm{G}}_{1}[1]\right)$, it follows that $\mathrm{G}\left(\widehat{\mathrm{G}}_{1}[1], \phi\right)$ has a unique Nash equilibrium, the gadget equilibrium $\widehat{\boldsymbol{\sigma}}$, which has, by Proposition 4.1, the following properties:

For each player $i \in[2]: \mathbf{U}_{i}(\widehat{\boldsymbol{\sigma}})=1$, so that $\sum_{i \in[2]} \mathrm{U}_{i}(\widehat{\boldsymbol{\sigma}})=2$; $\operatorname{Supp}\left(\widehat{\sigma}_{i}\right)=\widehat{\boldsymbol{\Sigma}}_{i}$, with $\left|\operatorname{Supp}\left(\widehat{\sigma}_{i}\right)\right|=1$, so that for each strategy $s \in \operatorname{Supp}\left(\sigma_{i}\right), \widehat{\sigma}_{i}(s)=1 ; \boldsymbol{\sigma}$ is uniform.

Hence, $\widetilde{G}$ has exactly three Nash equilibria, $\sigma^{1}, \sigma^{2}$ and $\sigma^{3}$, such that:

For each player $i \in[2]: \widetilde{U}_{i}\left(\boldsymbol{\sigma}^{1}\right)=\frac{1}{2}$, so that $\sum_{i \in[2]} \widetilde{U}_{i}\left(\boldsymbol{\sigma}^{1}\right)=1$; Supp $\left(\sigma_{i}^{1}\right)=\widehat{\Sigma}_{i} \cup \widehat{\Sigma}_{i}^{\prime}$, with $\left|\operatorname{Supp}\left(\widehat{\sigma}_{i}^{1}\right)\right|=2$; for each strategy $s \in \operatorname{Supp}\left(\sigma_{i}^{1}\right), \widehat{\sigma}_{i}^{1}(s)=\frac{1}{2} ; \boldsymbol{\sigma}^{1}$ is uniform.

For each $\boldsymbol{\sigma} \in\left\{\boldsymbol{\sigma}^{2}, \boldsymbol{\sigma}^{3}\right\}$ : For each player $i \in[2]$ : $\widetilde{\mathbf{U}}_{i}(\boldsymbol{\sigma})=1$, so that $\sum_{i \in[2]} \widetilde{U}_{i}(\boldsymbol{\sigma})=2$; either $\operatorname{Supp}\left(\sigma_{i}\right)=\widehat{\Sigma}_{i}$ or $\operatorname{Supp}\left(\sigma_{i}\right)=\widehat{\Sigma}_{i}^{\prime}$, with $\left|\operatorname{Supp}\left(\widehat{\sigma}_{i}\right)\right|=1$; for each strategy $s \in \operatorname{Supp}\left(\sigma_{i}\right), \widehat{\sigma}_{i}(s)=1 ; \boldsymbol{\sigma}$ is uniform.

Hence, when $\phi$ is unsatisfiable, each Nash equilibrium $\boldsymbol{\sigma}$ for $\widetilde{G}$ has the following properties: 
For each player $i \in[2]$ : (P.1) $\widetilde{U}_{i}(\boldsymbol{\sigma}) \geq \frac{1}{2}$, so that (P.2) $\sum_{i \in[2]} \widetilde{U}_{i}(\boldsymbol{\sigma}) \geq 1$; (P.3) $\operatorname{Supp}\left(\sigma_{i}\right) \subseteq \widehat{\Sigma}_{i} \cup \widehat{\Sigma}_{i}^{\prime}$, with (P.4) $\left|\operatorname{Supp}\left(\sigma_{i}\right)\right| \leq 2$; (P.5) for each strategy $s \in \operatorname{Supp}\left(\sigma_{i}\right)$, $\sigma_{i}(s) \geq \frac{1}{2} ;$ (P.6) $\sigma$ is uniform.

Assume now that $\phi$ is satisfiable. Then, by Proposition 5.14, the literal equilibrium $\boldsymbol{\sigma}=\boldsymbol{\sigma}(\gamma)$, for a satisfying assignement $\gamma$ of $\phi$, has the following properties:

For each player $i \in[2]: \mathbf{U}_{i}(\boldsymbol{\sigma})=\frac{2}{n}$, so that $\sum_{i \in[2]} \mathrm{U}_{i}(\boldsymbol{\sigma})=\frac{4}{n}$; $\operatorname{Supp}\left(\sigma_{i}\right)=\mathrm{L}$, with $\left|\operatorname{Supp}\left(\sigma_{i}\right)\right|=n$; for each strategy $s \in \operatorname{Supp}\left(\sigma_{i}\right), \sigma_{i}(s)=\frac{1}{n} ; \boldsymbol{\sigma}$ is uniform.

Here are the additional Nash equilibria for $\widetilde{G}$ and their properties:

- The $(\# \phi)^{2}$ balanced mixtures of literal equilibria for G:

For each player $i \in[2]: \widetilde{\mathbf{U}}_{i}\left(\boldsymbol{\sigma}^{4}\right)=\frac{1}{n}$, so that $\sum_{i \in[2]} \widetilde{\mathrm{U}}_{i}\left(\boldsymbol{\sigma}^{4}\right)=\frac{2}{n} ; \operatorname{Supp}\left(\sigma_{i}^{4}\right) \subset$ $\mathrm{L} \cup \mathrm{L}^{\prime}$, with $\left|\operatorname{Supp}\left(\sigma_{i}^{4}\right)\right|=2 n$; for each strategy $s \in \operatorname{Supp}\left(\sigma_{i}^{4}\right), \sigma_{i}^{4}(s)=\frac{1}{2 n} ; \boldsymbol{\sigma}^{4}$ is uniform.

For each $\boldsymbol{\sigma} \in\left\{\boldsymbol{\sigma}^{5}, \boldsymbol{\sigma}^{6}\right\}$ : For each player $i \in[2]$ : $\widetilde{\mathrm{U}}_{i}(\boldsymbol{\sigma})=\frac{2}{n}$, so that $\sum_{i \in[2]} \widetilde{\mathrm{U}}_{i}(\boldsymbol{\sigma})=$ $\frac{4}{n}$; Supp $\left(\sigma_{i}\right) \subset \mathrm{L} \cup \mathrm{L}^{\prime}$, with $\left|\operatorname{Supp}\left(\sigma_{i}\right)\right|=n$; for each strategy $s \in \operatorname{Supp}\left(\sigma_{i}\right)$, $\sigma_{i}(s)=\frac{1}{n} ; \boldsymbol{\sigma}$ is uniform.

- The $2 \cdot \# \phi$ balanced mixtures of a gadget equilibrium and a literal equilibrium for G: Note that

$$
\begin{aligned}
\boldsymbol{\sigma}^{7} & =\left\langle\frac{\frac{2}{n}}{1+\frac{2}{n}} \widehat{\sigma}_{1} \circ \frac{1}{1+\frac{2}{n}} \sigma_{2}, \frac{1}{1+\frac{2}{n}} \sigma_{1} \circ \frac{\frac{2}{n}}{1+\frac{2}{n}} \widehat{\sigma}_{2}\right\rangle \\
& =\left\langle\frac{2}{n+2} \widehat{\sigma}_{1} \circ \frac{n}{n+2} \sigma_{2}, \frac{n}{n+2} \sigma_{1} \circ \frac{2}{n+2} \widehat{\sigma}_{2}\right\rangle
\end{aligned}
$$

and

$$
\sigma^{8}=\left\langle\frac{n}{n+2} \sigma_{1} \circ \frac{2}{n+2} \widehat{\sigma}_{2}, \frac{2}{n+2} \widehat{\sigma}_{1} \circ \frac{n}{n+2} \sigma_{2}\right\rangle,
$$

respectively. Consider first $\boldsymbol{\sigma}^{7}=\boldsymbol{\sigma}^{7}(\gamma)$, for a satisfying assignment $\gamma$ of $\phi$. Then, by the definition of the win-lose GHR-symmetrization, the row (resp., column) player may get utility 1 only in two cases:

- The row player plays $\mathrm{L}^{\prime}$ and the column player plays L. By Proposition 5.14 (Condition (C.4)), for each literal $\ell \in \gamma, \sigma_{1}(\ell)=\sigma_{2}(\ell)=\frac{1}{n}$. Thus, by the balanced mixture $\boldsymbol{\sigma}^{7}$, for each player $i \in[2]$, for each literal $\ell \in \gamma$,

$$
\sigma_{i}^{7}(\ell)=\frac{n}{n+2} \cdot \sigma_{\bar{i}}(\ell)=\frac{n}{n+2} \cdot \frac{1}{n}=\frac{1}{n+2} .
$$


By the utility functions, there are two subcases in which the row (resp., column) player gets utility 1: The row player plays $\ell$, the column player plays $\ell^{\prime}$ with $\ell^{\prime} \neq \bar{\ell}$, and $I\left(\ell^{\prime}\right)-I(\ell) \in\{0,1\}$ (resp, $\left.I\left(\ell^{\prime}\right)-I(\ell) \in\{2,3\}\right)$. The two subcases occur with probability $\sum_{\ell, \ell^{\prime} \in \gamma \mid I\left(\ell^{\prime}\right)-I(\ell) \in\{0,1\}} \sigma_{1}(\ell) \cdot \sigma_{2}(\ell)=2 n \cdot\left(\frac{1}{n+2}\right)^{2}$ (resp., $\left.\sum_{\ell, \ell^{\prime} \in \gamma \mid I\left(\ell^{\prime}\right)-I(\ell) \in\{2,3\}} \sigma_{1}(\ell) \cdot \sigma_{2}(\ell)=2 n \cdot\left(\frac{1}{n+2}\right)^{2}\right)$.

- The row player plays $\widehat{\Sigma}_{1}$ and the column player plays $\widehat{\Sigma}_{2}^{\prime}$. Since each of $\widehat{\Sigma}_{1}$ and $\widehat{\Sigma}_{2}^{\prime}$ consists of a single strategy $s$, it follows, by Proposition 4.1 , that $\widehat{\sigma}_{1}(s)=\widehat{\sigma}_{2}(s)=1$. Thus, for each player $i \in[2]$,

$$
\sigma_{i}^{7}(s)=\frac{2}{n+2} \widehat{\sigma}_{\bar{i}}(s)=\frac{2}{n+2} .
$$

By the utility functions, there is a single subcase in which the row (resp., column) player gets utility 1: Both players choose $s$. The subcase occurs with probability $\left(\frac{2}{n+2}\right)^{2}$.

Hence, for each player $i \in[2]$,

$$
\widetilde{\mathbf{U}}_{i}\left(\boldsymbol{\sigma}^{7}\right)=2 n \cdot\left(\frac{1}{n+2}\right)^{2}+\left(\frac{2}{n+2}\right)^{2}=\frac{2}{n+2} .
$$

Since $\boldsymbol{\sigma}^{7}$ and $\boldsymbol{\sigma}^{8}$ form a symmetric pair of mixed profiles for the win-lose GHR-symmetrization $\widetilde{\mathrm{G}}$, it follows that for each player $i \in[2], \widetilde{\mathrm{U}}_{i}\left(\boldsymbol{\sigma}^{8}\right)=\frac{2}{n+2}$. Thus, we have:

For each $\boldsymbol{\sigma} \in\left\{\boldsymbol{\sigma}^{7}, \boldsymbol{\sigma}^{8}\right\}$ : For each player $i \in[2]$ : $\widetilde{\mathbf{U}}_{i}(\boldsymbol{\sigma})=\frac{2}{n+2}$, so that $\sum_{i \in[2]} \widetilde{U}_{i}(\boldsymbol{\sigma})=\frac{4}{n+2}$; either $\operatorname{Supp}\left(\sigma_{i}\right) \subset \widehat{\Sigma}_{i} \cup \mathrm{L} \cup \mathrm{L}^{\prime}$ or $\operatorname{Supp}\left(\sigma_{i}\right) \subset \widehat{\Sigma}_{i}^{\prime} \cup \mathrm{L} \cup \mathrm{L}^{\prime}$, with $\left|\operatorname{Supp}\left(\sigma_{i}\right)\right|=n+1$; for each strategy $s \in \operatorname{Supp}\left(\sigma_{i}\right), \sigma_{i}(s) \leq \frac{2}{n+2} ; \boldsymbol{\sigma}$ is non-uniform.

Hence, when $\phi$ is satisfiable, we have:

(1) Each additional Nash equilibrium $\boldsymbol{\sigma}$ among the $(\# \phi)^{2}$ balanced mixtures of literal equilibria for $\mathrm{G}$ has the following properties:

For each player $i \in[2]$ : (Q.1) $\widetilde{\mathbf{U}}_{i}(\boldsymbol{\sigma}) \leq \frac{2}{n} \leq \frac{2}{5}$, so that (Q.2) $\sum_{i \in[2]} \widetilde{U}_{i}(\boldsymbol{\sigma}) \leq$ $\frac{4}{n} \leq \frac{4}{5}$; (Q.3) Supp $\left(\sigma_{i}\right) \subset \mathrm{L} \cup \mathrm{L}^{\prime}$, with (Q.4) $\left|\operatorname{Supp}\left(\sigma_{i}\right)\right| \geq n \geq 5$; (Q.5) for each strategy $s \in \operatorname{Supp}\left(\sigma_{i}\right), \sigma_{i}(s)<\frac{2}{n+2} \leq \frac{2}{7} ;$ (Q.6) $\sigma$ is uniform.

(2) Each additional Nash equilibrium $\boldsymbol{\sigma}$ among the $2 \cdot \# \phi$ balanced mixtures of a gadget equilibrium and a literal equilibrium for $\mathrm{G}$ has the following properties: 
For each player $i \in[2]:(\mathrm{Q} .7) \widetilde{\mathrm{U}}_{i}(\boldsymbol{\sigma})<\frac{2}{n} \leq \frac{2}{5}$, so that (Q.8) $\sum_{i \in[2]} \widetilde{\mathrm{U}}_{i}(\boldsymbol{\sigma})<\frac{4}{n} \leq$ $\frac{4}{5}$; (Q.9) Supp $\left(\sigma_{i}\right) \subset \mathrm{L} \cup \mathrm{L}^{\prime} \cup \widehat{\Sigma}_{i} \cup \widehat{\Sigma}_{i}^{\prime}$, with (Q.10) $\left|\operatorname{Supp}\left(\sigma_{i}\right)\right|>n \geq 5$; (Q.11) for each strategy $s \in \operatorname{Supp}\left(\sigma_{i}\right), \sigma_{i}(s) \leq \frac{2}{n+2} \leq \frac{2}{7}$; (Q.12) $\boldsymbol{\sigma}$ is non-uniform.

Hence, we derive $\mathcal{N} \mathcal{P}$-hardness from the following table:

\begin{tabular}{|c|c|c|c|}
\hline \multirow{3}{*}{$\begin{array}{l}\mathcal{N} \mathcal{P} \text {-hard decision problem: } \\
\exists \text { NASH WITH SMALL UTILITIES, } \\
\text { with } \frac{2}{5} \leq u<\frac{1}{2} \\
\end{array}$} & \multicolumn{3}{|c|}{ By properties disentangling the satisfiability of $\phi$ : } \\
\hline & \multirow{2}{*}{$\begin{array}{l}\text { Unsat.: } \\
\text { (P.1) }\end{array}$} & \multicolumn{2}{|c|}{ Sat.: } \\
\hline & & (Q.1), (Q.7) & $\# \phi \cdot(\# \phi+2)$ \\
\hline $\begin{array}{l}\exists \text { NASH WITH SMALL TOTAL UTILITY, } \\
\text { with } \frac{4}{5} \leq u<1\end{array}$ & (P.2) & $(Q .2),(Q .8)$ & $\# \phi \cdot(\# \phi+2)$ \\
\hline $\begin{array}{l}\exists \text { NASH WITH LARGE SUPPORTS, } \\
\text { with } 2<k \leq 5\end{array}$ & (P.4) & (Q.4), (Q.10) & $\# \phi \cdot(\# \phi+2)$ \\
\hline $\begin{array}{l}\exists \text { NASH WITH RESTRICTING SUPPORTS, } \\
\text { with } T_{i}=\{\ell\} \text { and } T_{i}=\{\bar{\ell}\}, i \in[n] \text {, for any } \ell \in \mathrm{L}\end{array}$ & (P.3) & (Q.3), (Q.9) & $\# \phi \cdot(\# \phi+2)$ \\
\hline $\begin{array}{l}\exists \text { NASH WITH RESTRICTED SUPPORTS, } \\
\text { with } \mathrm{T}_{i}=\mathrm{L} \cup \mathrm{L}^{\prime}, i \in[n]\end{array}$ & (P.3) & (Q.3) & $(\# \phi)^{2}$ \\
\hline$\exists$ NASH WITH SMALL PROBABILITIES & (P.5) & $(\mathrm{Q} .5),(\mathrm{Q} .11)$ & $\# \phi \cdot(\# \phi+2)$ \\
\hline$\exists \neg$ UNIFORM NASH & (P.6) & (Q.12) & $2 \cdot \# \phi$ \\
\hline
\end{tabular}

The formulas in the rightmost column can be solved for $\# \phi$; by the $\# \mathcal{P}$-hardness of computing $\# \phi$ [35], this yields the \#P-hardness of the seven counting problems. Furthermore, except for $2 \cdot \# \phi$, these formulas preserve the parity $\oplus \phi$; by the $\oplus \mathcal{P}$-hardness of computing $\oplus \phi$ [33], this yields the $\oplus \mathcal{P}$-hardness of the six parity problems other than $\oplus \neg$ UNIFORM NASH.

Group II: Fix $\widehat{\mathrm{G}}:=\widehat{\mathrm{G}}_{3}$. Since $\kappa\left(\widehat{\mathrm{G}}_{3}\right)=4$, it follows that each of $\mathrm{G}\left(\widehat{\mathrm{G}}_{3}, \phi\right)$ and $\mathrm{GHR}(\mathrm{G})$ has size polynomial in the size of $\phi$. By Proposition 4.3, $\widehat{\mathrm{G}}_{3}$ has no uniform Nash equilibrium. Assume first that $\phi$ is unsatisfiable. Since $\mathcal{N E}\left(\mathrm{G}\left(\widehat{\mathrm{G}}_{3}, \phi\right)\right)=\mathcal{N} \mathcal{E}\left(\widehat{\mathrm{G}}_{3}\right)$, it follows that $\mathrm{G}\left(\widehat{\mathrm{G}}_{3}, \phi\right)$ has no uniform Nash equilibrium. Assume, by way of contradiction, that $\widetilde{G}=G H R(G)$ has a uniform Nash equilibrium $\boldsymbol{\sigma}$. By Proposition 6.6, exactly one of the following conditions holds for $\boldsymbol{\sigma}$ :

$\left(C^{\prime} .1\right) \overleftarrow{\sigma_{1}}, \overrightarrow{\sigma_{1}}, \overleftarrow{\sigma_{2}}, \overrightarrow{\sigma_{2}} \neq 0^{n}$, with $\sigma=\left\langle\overleftarrow{\varsigma_{1}}, \overrightarrow{\varsigma_{2}}\right\rangle *\left\langle\overleftarrow{\varsigma_{2}}, \overrightarrow{\varsigma_{1}}\right\rangle$ and $\left\langle\overleftarrow{\varsigma_{2}}, \overrightarrow{\varsigma_{1}}\right\rangle,\left\langle\overleftarrow{\varsigma_{1}}, \overrightarrow{\varsigma_{2}}\right\rangle \in \mathcal{N} \mathcal{E}(\mathrm{G})$

$\left(C^{\prime} .2\right) \overleftarrow{\sigma_{1}}=\overrightarrow{\sigma_{2}}=0^{n}$, with $\sigma=\left\langle 0^{n}, 0^{n}\right\rangle *\left\langle\overleftarrow{\varsigma_{2}}, \overrightarrow{\varsigma_{1}}\right\rangle$ and $\left\langle\overleftarrow{\varsigma_{2}}, \overrightarrow{\varsigma_{1}}\right\rangle \in \mathcal{N} \mathcal{E}(\mathrm{G})$

$\left(C^{\prime} .3\right) \overrightarrow{\sigma_{1}}=\overleftarrow{\sigma_{2}}=0^{n}$, with $\sigma=\left\langle 0^{n}, 0^{n}\right\rangle *\left\langle\overleftarrow{\varsigma_{1}}, \overrightarrow{\varsigma_{2}}\right\rangle$ and $\left\langle\overleftarrow{\varsigma_{1}}, \overrightarrow{\varsigma_{2}}\right\rangle \in \mathcal{N} \mathcal{E}(\mathrm{G})$

By Observation 6.3, the uniformity of $\boldsymbol{\sigma}$ implies that exactly one of these conditions holds:

$\left(C^{\prime \prime} .1\right) \overleftarrow{\sigma_{1}}, \overrightarrow{\sigma_{1}}, \overleftarrow{\sigma_{2}}, \overrightarrow{\sigma_{2}} \neq 0^{n}$, and both $\left\langle\overleftarrow{\varsigma_{2}}, \overrightarrow{\varsigma_{1}}\right\rangle$ and $\left\langle\overleftarrow{\varsigma_{1}}, \overrightarrow{\varsigma_{2}}\right\rangle$ are uniform Nash equilibria for G. 
$\left(C^{\prime \prime} .2\right) \overleftarrow{\sigma_{1}}=\overrightarrow{\sigma_{2}}=0^{n}$, and $\left\langle\overleftarrow{\varsigma_{2}}, \overrightarrow{\varsigma_{1}}\right\rangle$ is a uniform Nash equilibrium for G.

$\left(C^{\prime \prime} .3\right) \overrightarrow{\sigma_{1}}=\overleftarrow{\sigma_{2}}=0^{n}$, and $\left\langle\overleftarrow{\varsigma_{1}}, \overrightarrow{\varsigma_{2}}\right\rangle$ is a uniform Nash equilibrium for G.

So, in all cases, G has a uniform Nash equilibrium. A contradiction. This implies the following property: (P.1) $\widetilde{G}$ has no uniform Nash equilibrium.

Assume now that $\phi$ is satisfiable. Then, by Proposition 5.14 (Condition (4)), the literal equilibrium $\boldsymbol{\sigma}=\boldsymbol{\sigma}(\gamma)$, for a satisfying assignment $\gamma$ of $\phi$, is uniform. Here are the additional Nash equilibria for $\widetilde{G}$ and their properties:

- The $(\# \phi)^{2}$ balanced mixtures of literal equilibria for $\mathrm{G}: \sigma^{4}$ is uniform. By Observation 6.3, $\boldsymbol{\sigma}^{5}$ and $\boldsymbol{\sigma}^{6}$ are non-uniform.

- The $2 \cdot \# \phi$ balanced mixtures of a gadget equilibrium and a literal equilibrium for G: Since $\widehat{\boldsymbol{\sigma}}$ is non-uniform, it follows, by Observation 6.3, that $\boldsymbol{\sigma}^{7}$ and $\boldsymbol{\sigma}^{8}$ are non-uniform.

Hence, when $\phi$ is satisfiable, we have:

(1) Each additional Nash equilibrium $\boldsymbol{\sigma}$ among the $(\phi)^{2}$ balanced mixtures of literal equilibria for $\mathrm{G}$ has the following property: (Q.1) $\sigma$ is uniform.

(2) Each additional Nash equilibrium $\boldsymbol{\sigma}$ among the $2 \cdot \phi$ balanced mixtures of a gadget equilibrium and a literal equilibrium for $\mathrm{G}$ has the following property: (Q.2) $\sigma$ is non-uniform.

Hence, we derive $\mathcal{N} \mathcal{P}$-hardness from the following table:

\begin{tabular}{|l|l|l|l|}
\hline \hline $\mathcal{N} \mathcal{P}$-hard decision problem: & \multicolumn{3}{|l|}{ By properties disentangling the satisfiability of $\phi:$} \\
\cline { 2 - 4 } & Unsat.: & \multicolumn{1}{l|}{ Sat.: } \\
\hline \hline$\exists$ UNIFORM NASH & $($ P.1 $)$ & $($ Q.1) & $(\# \phi)^{2}$ \\
\hline \hline
\end{tabular}

The formula $(\# \phi)^{2}$ in the rightmost column can be solved for $\# \phi$; by the $\# \mathcal{P}$-hardness of computing $\# \phi$ [35], this yields the $\# \mathcal{P}$-hardness of the counting problem. Furthermore, the formula $(\# \phi)^{2}$ preserves the parity $\oplus \phi$; by the $\oplus \mathcal{P}$-hardness of computing $\oplus \phi$ [33], this yields the $\oplus \mathcal{P}$-hardness of the parity problem.

Group III: Fix $\widehat{\mathrm{G}}:=\widehat{\mathrm{G}}_{1}[h]$, where $h$ is polynomial in $n$ with $h>2 n$. Since $\kappa\left(\mathrm{G}_{1}[h]\right)=h$, where $h$ is polynomial in $n$, it follows that each of $\mathrm{G}=\mathrm{G}\left(\widehat{\mathrm{G}}_{1}[h], \phi\right)$ and $\widetilde{\mathrm{G}}=\mathrm{GHR}(\mathrm{G})$ has size polynomial in the size of $\phi$. By Proposition 4.1, $\widehat{G}_{1}[h]$ has a unique Nash equilibrium $\widehat{\boldsymbol{\sigma}}$. Assume first that $\phi$ is unsatisfiable. Since $\mathcal{N} \mathcal{E}\left(\mathrm{G}\left(\widehat{\mathrm{G}}_{1}[h], \phi\right)\right)=\mathcal{N} \mathcal{E}\left(\widehat{\mathrm{G}}_{1}[h]\right)$, it follows that $\mathrm{G}\left(\widehat{\mathrm{G}}_{1}[h], \phi\right)$ has a unique Nash equilibrium, the gadget equilibrium $\widehat{\boldsymbol{\sigma}}$, which has, by Proposition 4.1 , the following properties: 
For each player $i \in[2]: \mathbf{U}_{i}(\widehat{\boldsymbol{\sigma}})=\frac{1}{h}$, so that $\sum_{i \in[2]} \mathrm{U}_{i}(\widehat{\boldsymbol{\sigma}})=\frac{2}{h} ;\left|\operatorname{Supp}\left(\widehat{\sigma}_{i}\right)\right|=h$.

Hence, $\widetilde{G}$ has exactly three Nash equilbria, $\boldsymbol{\sigma}^{1}, \boldsymbol{\sigma}^{2}$ and $\boldsymbol{\sigma}^{3}$, such that:

For each player $i \in[2]: \widetilde{\mathbf{U}}_{i}\left(\boldsymbol{\sigma}^{1}\right)=\frac{1}{2 h}$, so that $\sum_{i \in[2]} \widetilde{\mathbf{U}}_{i}\left(\boldsymbol{\sigma}^{1}\right)=\frac{1}{h} ;\left|\operatorname{Supp}\left(\sigma_{i}^{1}\right)\right|=2 h$.

For each $\boldsymbol{\sigma} \in\left\{\boldsymbol{\sigma}^{2}, \boldsymbol{\sigma}^{3}\right\}$ : For each player $i \in[2]: \widetilde{\mathrm{U}}_{i}(\boldsymbol{\sigma})=\frac{1}{h}$, so that $\sum_{i \in[2]} \widetilde{\mathrm{U}}_{i}(\boldsymbol{\sigma})=$ $\frac{2}{h} ;\left|\operatorname{Supp}\left(\sigma_{i}\right)\right|=h$.

Hence, when $\phi$ is unsatisfiable, each Nash equilibrium $\boldsymbol{\sigma}$ for $\widetilde{G}$ has the following properties:

For each player $i \in[2]:(P .1) \widetilde{U}_{i}(\boldsymbol{\sigma}) \leq \frac{1}{h}<\frac{1}{2 n}$, so that (P.2) $\sum_{i \in[2]} \widetilde{U}_{i}(\boldsymbol{\sigma}) \leq \frac{2}{h}<\frac{1}{n}$;

(P.3) $\left|\operatorname{Supp}\left(\sigma_{i}\right)\right| \geq h>2 n$.

Assume now that $\phi$ is satisfiable. Then, by Proposition 5.14, the literal equilibrium $\sigma=$ $\boldsymbol{\sigma}(\gamma)$, for a satisfying assignment $\gamma$ of $\phi$, has the following properties:

For each player $i \in[2]: \mathbf{U}_{i}(\boldsymbol{\sigma})=\frac{2}{n}$, so that $\sum_{i \in[2]} \mathrm{U}_{i}(\boldsymbol{\sigma})=\frac{4}{n} ;\left|\operatorname{Supp}\left(\sigma_{i}\right)\right|=n$.

Here are the additional Nash equilibria of $\widetilde{G}$ and their properties:

- The $(\# \phi)^{2}$ balanced mixtures of literal equilibria for $\mathrm{G}$ :

For each player $i \in[2]: \widetilde{\mathbf{U}}_{i}\left(\boldsymbol{\sigma}^{4}\right)=\frac{1}{n}$, so that $\sum_{i \in[2]} \widetilde{\mathbf{U}}_{i}\left(\boldsymbol{\sigma}^{4}\right)=\frac{2}{n} ;\left|\operatorname{Supp}\left(\sigma_{i}^{4}\right)\right|=$ $2 n$.

For each $\boldsymbol{\sigma} \in\left\{\boldsymbol{\sigma}^{5}, \boldsymbol{\sigma}^{6}\right\}:$ For each player $i \in[2]: \widetilde{\mathrm{U}}_{i}(\boldsymbol{\sigma})=\frac{2}{n}$, so that $\sum_{i \in[2]} \widetilde{\mathrm{U}}_{i}(\boldsymbol{\sigma})=$ $\frac{4}{n} ;\left|\operatorname{Supp}\left(\sigma_{i}\right)\right|=n$.

- The $2 \cdot \# \phi$ balanced mixtures of a gadget equilibrium and a literal equilibrium for G: Note that

$$
\begin{aligned}
\boldsymbol{\sigma}^{7} & =\left\langle\frac{\frac{2}{n}}{\frac{1}{h}+\frac{2}{n}} \widehat{\sigma}_{1} \circ \frac{\frac{1}{h}}{\frac{1}{h}+\frac{2}{n}} \sigma_{2}, \frac{\frac{1}{h}}{\frac{1}{h}+\frac{2}{n}} \sigma_{1} \circ \frac{\frac{2}{n}}{\frac{1}{h}+\frac{2}{n}} \widehat{\sigma}_{2}\right\rangle \\
& =\left\langle\frac{2 h}{n+2 h} \widehat{\sigma}_{1} \circ \frac{n}{n+2 h} \sigma_{2}, \frac{n}{n+2 h} \sigma_{1} \circ \frac{2 h}{n+2 h} \widehat{\sigma}_{2}\right\rangle,
\end{aligned}
$$

and

$$
\boldsymbol{\sigma}^{8}=\left\langle\frac{n}{n+2 h} \sigma_{1} \circ \frac{2 h}{n+2 h} \widehat{\sigma}_{2}, \frac{2 h}{n+2 h} \widehat{\sigma}_{1} \circ \frac{n}{n+2 h} \sigma_{2}\right\rangle,
$$

respectively. Consider first $\boldsymbol{\sigma}^{7}$, with $\boldsymbol{\sigma}^{7}=\boldsymbol{\sigma}^{7}(\gamma)$ for a satisfying assignment $\gamma$ of $\phi$. Then, by the definition of the win-lose GHR-symmetrization, the row (resp., column) player may get utility 1 only in the following two cases: 
- The row player plays $\mathrm{L}^{\prime}$ and the column player plays L. By Proposition 5.14 (Condition (C.4)), for each literal $\ell \in \gamma, \sigma_{1}(\ell)=\sigma_{2}(\ell)=\frac{1}{n}$. Thus, by the balanced mixture $\boldsymbol{\sigma}^{7}$, for each player $i \in[2]$, for each literal $\ell \in \gamma$,

$$
\sigma_{i}^{7}(\ell)=\frac{n}{n+2 h} \cdot \sigma_{\bar{i}}(\ell)=\frac{n}{n+2 h} \cdot \frac{1}{n}=\frac{1}{n+2 h} .
$$

By the utility functions, there are two subcases in which the row (resp., column) player gets utility 1: The row player chooses $\ell$, the column player chooses $\ell^{\prime}$ with $\ell^{\prime} \neq \bar{\ell}$, and $I\left(\ell^{\prime}\right)-I(\ell) \in\{0,1\}$ (resp, $\left.I\left(\ell^{\prime}\right)-I(\ell) \in\{2,3\}\right)$. The two subcases occur with probability $\sum_{\ell, \ell^{\prime} \in \gamma \mid I\left(\ell^{\prime}\right)-I(\ell) \in\{0,1\}} \sigma_{1}(\ell) \cdot \sigma_{2}(\ell)=2 n \cdot\left(\frac{1}{n+2 h}\right)^{2} \cdot$ (resp., $\left.\sum_{\ell, \ell^{\prime} \in \gamma \mid I\left(\ell^{\prime}\right)-I(\ell) \in\{2,3\}} \sigma_{1}(\ell) \cdot \sigma_{2}(\ell)=2 n \cdot\left(\frac{1}{n+2 h}\right)^{2}\right)$.

- The row player plays $\widehat{\Sigma}_{1}$ and the column player plays $\widehat{\Sigma}_{2}^{\prime}$. By Proposition 4.1, it follows that for each strategy $s \in[h], \widehat{\sigma}_{1}(s)=\widehat{\sigma}_{2}(s)=\frac{1}{h}$. Thus, by the balanced mixture $\boldsymbol{\sigma}^{7}$, for each player $i \in[2]$, for each strategy $s \in[h], \sigma_{i}^{7}(s)=\frac{2 h}{n+2 h} \widehat{\sigma}_{\bar{i}}(s)=$ $\frac{2}{n+2 h}$. By the utility functions, there is a single subcase in which the row (resp., column) player gets utility 1: The row player chooses $s_{l}$ and the column player chooses $s_{l}$ (resp., the row player chooses $s_{l}$ and the column player chooses $s_{l+1}$ ). The subcase occurs with probability $\sum_{s_{l} \in[h]} \sigma_{1}^{7}\left(s_{l}\right) \cdot \sigma_{2}^{7}\left(s_{l}\right)=h \cdot\left(\frac{2}{n+2 h}\right)^{2}$ (resp., $\left.\sum_{s_{l} \in[h]} \sigma_{1}^{7}\left(s_{l}\right) \cdot \sigma_{2}^{7}\left(s_{l+1}\right)=h \cdot\left(\frac{2}{n+2 h}\right)^{2}\right)$.

It follows that for each player $i \in[2]$,

$$
\widetilde{\mathrm{U}}_{i}\left(\boldsymbol{\sigma}^{7}\right)=2 n \cdot\left(\frac{1}{n+2 h}\right)^{2}+h \cdot\left(\frac{2}{n+2 h}\right)^{2}=\frac{2}{n+2 h} .
$$

Since $\boldsymbol{\sigma}^{7}$ and $\boldsymbol{\sigma}^{8}$ form a symmetric pair of mixed profiles for the win-lose GHR-symmetrization $\widetilde{\mathrm{G}}$, it follows that for each player $i \in[2], \widetilde{\mathrm{U}}_{i}\left(\boldsymbol{\sigma}^{8}\right)=\frac{2}{n+2 h}$. Thus, it follows:

For each $\boldsymbol{\sigma} \in\left\{\boldsymbol{\sigma}^{7}, \boldsymbol{\sigma}^{8}\right\}$ : For each player $i \in[2]$ : $\widetilde{\mathrm{U}}_{i}(\boldsymbol{\sigma})=\frac{2}{n+2 h}$, so that $\sum_{i \in[2]} \widetilde{U}_{i}(\boldsymbol{\sigma})=\frac{4}{n+2 h} ;\left|\operatorname{Supp}\left(\sigma_{i}\right)\right|=n+h$.

Hence, when $\phi$ is satisfiable, we have:

(1) Each additional Nash equilibrium among the $(\# \phi)^{2}$ balanced mixtures of literal equilibria for $\mathrm{G}$ has the following properties:

For each player $i \in[2]:(\mathrm{Q} .1) \widetilde{\mathrm{U}}_{i}(\boldsymbol{\sigma}) \geq \frac{1}{n}$, so that $(\mathrm{Q} .2) \sum_{i \in[2]} \widetilde{\mathrm{U}}_{i}(\boldsymbol{\sigma}) \geq \frac{2}{n} ;(\mathrm{Q} .3)$ $\operatorname{Supp}\left(\sigma_{i}\right) \leq 2 n<h$.

(2) Each additional Nash equilibrium among the $2 \cdot \# \phi$ balanced mixtures of a gadget equilibrium and a literal equilibrium for $\mathrm{G}$ has the following properties: 
For each player $i \in[2]:(\mathrm{Q} .4) \widetilde{\mathrm{U}}_{i}(\boldsymbol{\sigma})=\frac{2}{n+2 h}$, so that $(\mathrm{Q} .5) \sum_{i \in[2]} \widetilde{\mathrm{U}}_{i}(\boldsymbol{\sigma})=$ $\frac{4}{n+2 h} ;$ (Q.6) $\operatorname{Supp}\left(\sigma_{i}\right)=n+h$.

Note that the choice $h>2 n$ implies that $\frac{2}{n+2 h}<\frac{1}{2 h}$ and $h<n+h<2 h$. Hence, the properties (Q.4), (Q.5) and (Q.6) are not contradictory to the properties (P.1), (P.2) and (P.3), respectively, of Nash equilibria, holding when $\phi$ is unsatisfiable.

Hence, we derive $\mathcal{N} \mathcal{P}$-hardness from the following table:

\begin{tabular}{|l|l|l|l|}
\hline \hline \multirow{2}{*}{$\mathcal{N}$ P-hard decision problem: } & \multicolumn{3}{|l|}{ By properties disentangling the satisfiability of $\phi:$} \\
\cline { 2 - 4 } & Unsat.: & \multicolumn{2}{|l|}{ Sat.: } \\
\hline \hline $\begin{array}{l}\exists \text { NASH WITH LARGE UTILITIES, } \\
\text { with } \frac{1}{2 n} \leq u<\frac{1}{n}\end{array}$ & (P.1) & (Q.1) & $(\# \phi)^{2}$ \\
\hline $\begin{array}{l}\exists \text { NASH WITH LARGE TOTAL UTILITY, } \\
\text { with } \frac{1}{n} \leq u<\frac{2}{n}\end{array}$ & (P.2) & $($ Q.2) & $(\# \phi)^{2}$ \\
\hline $\begin{array}{l}\exists \text { NASH WITH SMALL SUPPORTS, } \\
\text { with } 2 n \leq k<h\end{array}$ & (P.3) & $($ Q.3) & $(\# \phi)^{2}$ \\
\hline \hline
\end{tabular}

The formula $(\# \phi)^{2}$ in the rightmost column can be solved for $\# \phi$; by the $\# \mathcal{P}$-hardness of computing $\# \phi$ [35], this yields the $\# \mathcal{P}$-hardness of the three counting problems. Furthermore, this formula preserves the parity $\oplus \phi$; by the $\oplus \mathcal{P}$-hardness of computing $\oplus \phi[33$, this yields the $\oplus \mathcal{P}$-hardness of the three parity problems.

Group IV: We start with an informal outline of the proof. First note that the problems $\exists 2$ $\overline{\mathrm{NASH}}$ and $\exists 3 \mathrm{NASH}$ "escape" the technique using the GHR-symmetrization of $\mathrm{G}=\mathrm{G}(\widehat{\mathrm{G}}, \phi)$ since, by Theorem 6.6, each Nash equilibrium of $G$ gives rise to exactly three, distinct Nash equilibria of $\widetilde{G}=\operatorname{GHR}(G)$; thus, even if $\widehat{G}$ had a unique Nash equilibrium, this approach could only help proving the $\mathcal{N} \mathcal{P}$-hardness of $\exists k+1 \mathrm{NASH}$ with $k \geq 3$. Instead, to prove the $\mathcal{N} \mathcal{P}$ hardness of $\exists k+1 \mathrm{NASH}$ for all integers $k \geq 1$, we "embed" the win-lose diagonal game $\widehat{\mathrm{G}}_{5}[k]$ (Section 4.5) as a subgame of $\widetilde{\mathrm{G}}$. Denote the resulting game as $\widetilde{\mathrm{G}} \| \widehat{\mathrm{G}}_{5}[k]$, which is still symmetric and win-lose. We shall establish that when $\phi$ is unsatisfiable, all Nash equilibria of $\widetilde{G}$ which are "inherited" from $\widehat{G}$ are "destroyed" in $\widetilde{G} \| \widehat{G}_{5}[k]$ as long as $\widehat{G}$ has been chosen so that the players' expected utilities are smaller than 1 in a Nash equilibrium; thus, in this case, $\widetilde{\mathrm{G}} \| \widehat{\mathrm{G}}_{5}[k]$ has exactly $k$ Nash equilibria, which are "inherited" from the win-lose diagonal game $\widehat{\mathrm{G}}_{5}[k]$; instead, when $\phi$ is satisfiable, all $k$ Nash equilibria inherited from $\widehat{\mathrm{G}}_{5}[k]$ survive, and a new Nash equilibrium is created for each satisfying assignment of $\phi$. Besides the decision problem $\exists k+1 \mathrm{NASH}$, with $k \geq 1$, the same idea goes through for the last three decision problems in Group $I V$. We now continue with the details of the formal proof.

Fix $\widehat{G}:=\widehat{G}_{1}[2]$. Since $\kappa\left(\widehat{G}_{1}[2]\right)=2$, it follows that each of $\mathrm{G}$ and $\widetilde{\mathrm{G}}$ has size polynomial in the size of $\phi$. Denote as $\widetilde{G} \| \widehat{G}_{5}[k]$ the win-lose game resulting from $\widetilde{G}$ by adding the $k$ strategies 
from $\mathrm{T}:=\left\{t_{1}, \ldots, t_{k}\right\}$ to the strategy set of each player, so that $\Sigma\left(\widetilde{\mathrm{G}} \| \widehat{\mathrm{G}}_{5}[k]\right)=\Sigma(\widetilde{\mathrm{G}}) \cup \mathrm{T}$, and setting:

(C.1) $\mathrm{U}(\langle s, t\rangle):=\langle 0,1\rangle$ and $\mathrm{U}(\langle t, s\rangle):=\langle 1,0\rangle$, when $s \in \Sigma(\widetilde{\mathrm{G}}) \backslash\left(\mathrm{L} \cup \mathrm{L}^{\prime}\right)$ and $t \in \mathrm{T}$.

(C.2) $\mathrm{U}(\langle s, t\rangle):=\mathrm{U}(\langle t, s\rangle):=\langle 0,0\rangle$, when $s \in \mathrm{L} \cup \mathrm{L}^{\prime}$ and $t \in \mathrm{T}$.

(C.3) $\mathrm{U}\left(\left\langle t_{j}, t_{l}\right\rangle\right):=\left\langle\mathrm{D}_{k}[j, l], \mathrm{D}_{k}^{\mathrm{T}}[j, l]\right\rangle$, when $j, l \in[k]$; thus, $\widehat{\mathrm{G}}_{5}[k]$ is "embedded" into $\widetilde{\mathrm{G}}$ as a subgame.

Note that $\widetilde{G} \| \widehat{G}_{5}[k]$ is a symmetric game, which has size polynomial in the size of $\phi$. Furthermore, by Proposition 4.10, for each Nash equilibrium $\boldsymbol{\sigma} \in \mathcal{N} \mathcal{E}\left(\widehat{\mathrm{G}}_{5}[k]\right)$, for each player $i \in[2]$, $\mathrm{U}_{i}(\boldsymbol{\sigma})=1$. Since $\widetilde{\mathrm{G}} \| \widehat{\mathrm{G}}_{5}[k]$ is win-lose and $\mathrm{T} \subseteq \Sigma\left(\widetilde{\mathrm{G}} \| \widehat{\mathrm{G}}_{5}[k]\right)$, it follows that $\boldsymbol{\sigma}$ is a Nash equilibrium for $\widetilde{G} \| \widehat{G}_{5}[k]$. Thus, $\mathcal{N} \mathcal{E}\left(\widehat{G}_{5}[k]\right) \subseteq \mathcal{N} \mathcal{E}\left(\widetilde{\mathrm{G}} \| \widehat{\mathrm{G}}_{5}[k]\right)$. We continue to prove two simple properties of the Nash equilibria for $\widetilde{G} \| \widehat{G}_{5}[k]$.

Claim 7.2 Fix a Nash equilibrium $\boldsymbol{\sigma} \in \mathcal{N} \mathcal{E}\left(\widetilde{\mathrm{G}} \| \widehat{\mathrm{G}}_{5}[k]\right)$ with $\operatorname{Supp}\left(\sigma_{i}\right) \subseteq \mathrm{T}$ for some player $i \in[2]$. Then, $\boldsymbol{\sigma} \in \mathcal{N} \mathcal{E}\left(\widehat{\mathrm{G}}_{5}[k]\right)$.

Proof: By Cases (C.1) and (C.2) from the definition of the utility functions of $\widetilde{\mathrm{G}}_{\|} \widehat{\mathrm{G}}_{5}[k]$, $\mathrm{U}_{\bar{i}}\left(\boldsymbol{\sigma}_{-\bar{i}} \diamond s\right)=0$ for each strategy $s \in \Sigma(\widetilde{\mathrm{G}})$. Since $\widehat{\mathrm{G}}_{5}[k]$ has the positive utility property, there is a strategy $t \in \mathrm{T}$ such that $\mathrm{U}_{\bar{i}}\left(\boldsymbol{\sigma}_{-\bar{i}} \diamond t\right)>0$. Thus, by Lemma 2.1, every best-response strategy for player $\bar{i}$ is contained in T, which implies that $\operatorname{Supp}\left(\sigma_{\bar{i}}\right) \subseteq \mathrm{T}$. Hence, $\sigma$ maps to a mixed profile for $\widehat{G}_{5}[k]$, and the claim follows.

Fix now a mixed profile $\boldsymbol{\sigma}$ for $\widetilde{\mathrm{G}} \| \widehat{\mathrm{G}}_{5}[k]$ such that for each player $i \in[2], \sigma_{i}(\mathrm{~T})<1$; so, for each player $i \in[2], \operatorname{Supp}\left(\sigma_{i}\right) \nsubseteq \mathrm{T}$. Construct from $\boldsymbol{\sigma}$ the mixed profile $\boldsymbol{\varsigma}=\boldsymbol{\varsigma}(\boldsymbol{\sigma})$ for $\widetilde{\mathrm{G}}$ such that for each player $i \in[2]$ and strategy $s \in \Sigma(\widetilde{G})$,

$$
\varsigma_{i}(s):=\frac{\sigma_{i}(s)}{1-\sigma_{i}(\mathbf{T})} ;
$$

that is, $\varsigma$ is the projection of $\boldsymbol{\sigma}$ to $\Sigma(\widetilde{\mathrm{G}})$. Note that, by the construction, for each player $i \in[2]$, $\operatorname{Supp}\left(\varsigma_{i}\right) \subseteq \operatorname{Supp}\left(\sigma_{i}\right)$. We now prove:

Claim 7.3 Fix a Nash equilibrium $\boldsymbol{\sigma} \in \mathcal{N} \mathcal{E}\left(\widetilde{\mathrm{G}} \| \widehat{\mathrm{G}}_{5}[k]\right) \backslash \mathcal{N E}\left(\widehat{\mathrm{G}}_{5}[k]\right)$. Then, $\boldsymbol{\varsigma}(\boldsymbol{\sigma})$ is a Nash equilibrium for $\widetilde{\mathrm{G}}$.

Proof: Assume, by way of contradiction, that $\boldsymbol{\varsigma}(\boldsymbol{\sigma})$ is not a Nash equilibrium for $\widetilde{G}$. Then, by Lemma 2.1, there is a player $i \in[2]$ and a pair of strategies $s^{\prime} \in \Sigma(\widetilde{G})$ and $s \in \operatorname{Supp}\left(\varsigma_{i}\right)$ such that

$$
\mathrm{U}_{i}\left(\varsigma_{-i} \diamond s^{\prime}\right)>\mathrm{U}_{i}\left(\varsigma_{-i} \diamond s\right)
$$


Since $\operatorname{Supp}\left(\varsigma_{i}\right) \subseteq \operatorname{Supp}\left(\sigma_{i}\right)$, it follows that $s \in \operatorname{Supp}\left(\sigma_{i}\right)$. Since $\widetilde{\mathrm{G}} \| \widehat{\mathrm{G}}_{5}[k]$ is a symmetric game, we may assume, without loss of generality, that $i=1$. Clearly,

$$
\begin{aligned}
\mathrm{U}_{1}\left(\varsigma_{-1} \diamond s^{\prime}\right) & =\sum_{t \in \Sigma(\widetilde{\mathrm{G}})} \mathrm{U}_{1}\left(\left\langle s^{\prime}, t\right\rangle\right) \cdot \varsigma_{2}(t) \\
& =\frac{1}{1-\sigma_{2}(\mathrm{~T})} \sum_{t \in \Sigma(\widetilde{\mathrm{G}})} \mathrm{U}_{1}\left(\left\langle s^{\prime}, t\right\rangle\right) \cdot \sigma_{2}(t),
\end{aligned}
$$

and

$$
\begin{aligned}
\mathrm{U}_{1}\left(\boldsymbol{\varsigma}_{-1} \diamond s\right) & =\sum_{t \in \Sigma(\widetilde{\mathrm{G}})} \mathrm{U}_{1}(\langle s, t\rangle) \cdot \varsigma_{2}(t) \\
& =\frac{1}{1-\sigma_{2}(\mathrm{~T})} \sum_{t \in \Sigma(\widehat{\mathrm{G}})} \mathrm{U}_{1}(\langle s, t\rangle) \cdot \sigma_{2}(t) .
\end{aligned}
$$

Hence, by (9), it follows that

$$
\sum_{t \in \Sigma(\widehat{\mathrm{G}})} \mathrm{U}_{1}\left(\left\langle s^{\prime}, t\right\rangle\right) \cdot \sigma_{2}(t)>\sum_{t \in \Sigma(\widehat{\mathrm{G}})} \mathrm{U}_{1}(\langle s, t\rangle) \cdot \sigma_{2}(t) .
$$

Thus,

$$
\begin{aligned}
& \mathrm{U}_{1}\left(\boldsymbol{\sigma}_{-1} \diamond s^{\prime}\right) \\
& =\sum_{t \in \Sigma\left(\widetilde{\mathrm{G}} \| \widehat{\mathrm{G}}_{5}[k]\right)} \mathrm{U}_{1}\left(\left\langle s^{\prime}, t\right\rangle\right) \cdot \sigma_{2}(t) \\
& =\quad \sum_{t \in \Sigma(\widetilde{\mathrm{G}})} \mathrm{U}_{1}\left(\left\langle s^{\prime}, t\right\rangle\right) \cdot \sigma_{2}(t) \quad\left(\operatorname{since} \Sigma\left(\widetilde{\mathrm{G}} \| \widehat{\mathrm{G}}_{5}[k]\right)=\Sigma(\widetilde{\mathrm{G}}) \cup \mathrm{T} \& \mathrm{U}_{1}(s, t)=0 \text { for } s \in \Sigma(\widetilde{\mathrm{G}}), t \in \mathrm{T}\right) \\
& >\quad \sum_{t \in \Sigma(\widetilde{\mathrm{G}})} \mathrm{U}_{1}(\langle s, t\rangle) \cdot \sigma_{2}(t) \quad(\text { by (10) }) \\
& =\sum_{t \in \Sigma\left(\widetilde{\mathrm{G}} \| \widehat{\mathrm{G}}_{5}[k]\right)} \mathrm{U}_{1}(\langle s, t\rangle) \cdot \sigma_{2}(t) \quad\left(\text { since } \Sigma\left(\widetilde{\mathrm{G}} \| \widehat{\mathrm{G}}_{5}[k]\right)=\Sigma(\widetilde{\mathrm{G}}) \cup \mathrm{T} \& \mathrm{U}_{1}(s, t)=0 \text { for } s \in \Sigma(\widetilde{\mathrm{G}}), t \in \mathrm{T}\right) \\
& =\quad \mathrm{U}_{1}\left(\boldsymbol{\sigma}_{-1} \diamond s\right) \text {. }
\end{aligned}
$$

Since $\boldsymbol{\sigma}$ is a Nash equilibrium for $\widetilde{\mathrm{G}} \| \widehat{\mathrm{G}}_{5}[k]$ and $s \in \operatorname{Supp}\left(\sigma_{1}\right)$, Lemma 2.1 (Condition (1)) implies that $\mathrm{U}_{1}\left(\boldsymbol{\sigma}_{-1} \diamond s\right) \geq \mathbf{U}_{1}\left(\boldsymbol{\sigma}_{-1} \diamond s^{\prime}\right)$. A contradiction.

We now prove:

Lemma 7.4 Assume that $\phi$ is unsatisfiable. Then, $\mathcal{N E}\left(\widetilde{\mathrm{G}} \| \widehat{\mathrm{G}}_{5}[k]\right)=\mathcal{N} \mathcal{E}\left(\widehat{\mathrm{G}}_{5}[k]\right)$.

Proof: $\quad$ Since $\mathcal{N E}\left(\widehat{\mathrm{G}}_{5}[k]\right) \subseteq \mathcal{N} \mathcal{E}\left(\widetilde{\mathrm{G}} \| \widehat{\mathrm{G}}_{5}[k]\right)$, it remains to prove that $\mathcal{N} \mathcal{E}\left(\widetilde{\mathrm{G}} \| \widehat{\mathrm{G}}_{5}[k]\right) \backslash \mathcal{N} \mathcal{E}\left(\widehat{\mathrm{G}}_{5}[k]\right)=$ $\emptyset$. Assume, by way of contradiction, that there is a Nash equilibrium $\boldsymbol{\sigma} \in \mathcal{N} \mathcal{E}\left(\widetilde{\mathrm{G}}_{\|} \| \widehat{\mathrm{G}}_{5}[k]\right) \backslash$ $\mathcal{N E}\left(\widehat{\mathrm{G}}_{5}[k]\right)$.

Since $\phi$ is unsatisfiable, it follows, by Proposition 5.13, that $\mathcal{N E}(G)=\mathcal{N E}\left(\widehat{G}_{1}[2]\right)$. Thus, by Proposition 6.6, for each $\boldsymbol{\tau} \in \mathcal{N} \mathcal{E}(\widetilde{\mathrm{G}})$, for each player $i \in[2]$, Supp $\left(\tau_{i}\right) \subseteq \widehat{\Sigma}_{i} \cup \widehat{\Sigma}_{i}^{\prime}$. Since, by Claim 7.3. $\varsigma(\boldsymbol{\sigma})$ is a Nash equilibrium for $\widetilde{G}$, it follows that for each player $i \in[2], \operatorname{Supp}\left(\sigma_{i}\right) \subseteq \widehat{\Sigma}_{i} \cup \widehat{\Sigma}_{i}^{\prime} \cup \mathrm{T}$. Since $\boldsymbol{\sigma} \notin \mathcal{N} \mathcal{E}\left(\widehat{\mathrm{G}}_{5}[k]\right)$, Claim 7.2 implies that for each player $i \in[2], \operatorname{Supp}\left(\sigma_{i}\right) \nsubseteq \mathrm{T}$. Hence, it follows that for each player $i \in[2], \operatorname{Supp}\left(\sigma_{i}\right) \cap\left(\widehat{\Sigma}_{i} \cup \widehat{\Sigma}_{i}^{\prime}\right) \neq \emptyset$. There are two cases: 
1. Assume first that for each player $i \in[2], \operatorname{Supp}\left(\sigma_{i}\right) \subseteq \widehat{\Sigma}_{i} \cup \widehat{\Sigma}_{i}^{\prime}$. By the utility functions of $\widehat{G}_{1}[2]$ and by the definition of the GHR-symmetrization, it follows that for any profile $\mathbf{s}$ supported in $\boldsymbol{\sigma}, \mathrm{U}_{1}(\mathbf{s})+\mathrm{U}_{2}(\mathbf{s}) \leq 1$. This implies that there is a player $i \in[2]$ with $\mathrm{U}_{i}(\mathbf{s})<1 * *$ This implies that $\mathrm{U}_{i}(\boldsymbol{\sigma})<1$. Consider now player $i$ switching to a pure strategy $t \in \mathrm{T}$. Note that only profiles falling into case (C.1) are supported in $\sigma_{-i} \diamond t$. Hence, by the utility functions of $\widetilde{G} \| \widehat{G}_{5}[k], \mathbf{U}_{i}\left(\boldsymbol{\sigma}_{-i} \diamond t\right)=1$. Since $\mathbf{U}_{i}(\boldsymbol{\sigma})<1$, Lemma 2.1 (Condition (1)) yields a contradiction.

2. Assume now that there is a player $i \in[2]$ with $\operatorname{Supp}\left(\sigma_{i}\right) \cap T \neq \emptyset$. Since $\widetilde{\mathrm{G}}_{\|} \widehat{\mathrm{G}}_{5}[k]$ is a symmetric game, we may assume, without loss of generality, that $i=2$. Since $\operatorname{Supp}\left(\sigma_{i}\right) \cap\left(\widehat{\Sigma}_{i} \cup \widehat{\Sigma}_{i}^{\prime}\right) \neq \emptyset$ for each player $i \in[2]$, there is a strategy $s \in \operatorname{Supp}\left(\sigma_{1}\right) \cap\left(\widehat{\Sigma}_{1} \cup \widehat{\Sigma}_{1}^{\prime}\right)$. Thus,

$$
\begin{array}{rlr} 
& \mathrm{U}_{1}\left(\boldsymbol{\sigma}_{-1} \diamond s\right) \\
= & \sum_{s^{\prime} \in \widehat{\Sigma}_{2} \cup \widehat{\Sigma}_{2}^{\prime} \mathrm{U}_{1}\left(\left\langle s, s^{\prime}\right\rangle\right) \cdot \sigma_{2}\left(s^{\prime}\right)+\sum_{t^{\prime} \in \mathrm{T}} \mathrm{U}_{1}\left(\left\langle s, t^{\prime}\right\rangle\right) \cdot \sigma_{2}\left(t^{\prime}\right)} & \\
= & \sum_{s^{\prime} \in \widehat{\Sigma}_{2} \cup \widehat{\Sigma}_{2}^{\prime}} \mathrm{U}_{1}\left(\left\langle s, s^{\prime}\right\rangle\right) \cdot \sigma_{2}\left(s^{\prime}\right) & \text { (from Case (C.1) in the utility functions) } \\
\leq & \sum_{s^{\prime} \in \widehat{\Sigma}_{2} \cup \widehat{\Sigma}_{2}^{\prime}} \sigma_{2}\left(s^{\prime}\right) & \text { (since } \widetilde{\mathrm{G}} \| \widehat{\mathrm{G}}_{5}[k] \text { is win-lose). }
\end{array}
$$

Since $\widehat{\mathrm{G}}_{5}[k]$ has the positive utility property and $\operatorname{Supp}\left(\sigma_{2}\right) \cap \mathrm{T} \neq \emptyset$, it follows that there is a strategy $t \in \mathrm{T}$ such that

$$
\sum_{t^{\prime} \in \mathrm{T}} \mathrm{U}_{1}\left(\left\langle t, t^{\prime}\right\rangle\right) \cdot \sigma_{2}\left(t^{\prime}\right)>0
$$

So consider player 1 switching to the pure strategy $t$. Then,

$$
\begin{array}{rlr} 
& \mathrm{U}_{1}\left(\boldsymbol{\sigma}_{-1} \diamond t\right) \\
= & \sum_{s^{\prime} \in \widehat{\Sigma}_{2} \cup \widehat{\Sigma}_{2}^{\prime}} \mathrm{U}_{1}\left(\left\langle t, s^{\prime}\right\rangle\right) \cdot \sigma_{2}\left(s^{\prime}\right)+\sum_{t^{\prime} \in \mathrm{T}} \mathrm{U}_{1}\left(\left\langle t, t^{\prime}\right\rangle\right) \cdot \sigma_{2}\left(t^{\prime}\right) & \\
> & \sum_{s^{\prime} \in \widehat{\Sigma}_{2} \cup \widehat{\Sigma}_{2}^{\prime}} \mathrm{U}_{1}\left(\left\langle t, s^{\prime}\right\rangle\right) \cdot \sigma_{2}\left(s^{\prime}\right) & \text { (from (111) }) \\
= & \sum_{s^{\prime} \in \widehat{\Sigma}_{2} \cup \widehat{\Sigma}_{2}^{\prime}} \sigma_{2}\left(s^{\prime}\right) & \text { (from Case (C.1) in the utility functions). }
\end{array}
$$

It follows that

$$
\mathrm{U}_{1}\left(\sigma_{-1} \diamond s\right)<\mathrm{U}_{1}\left(\boldsymbol{\sigma}_{-1} \diamond t\right) .
$$

Since $s \in \operatorname{Supp}\left(\sigma_{1}\right)$, Lemma 2.1 (Condition (1)) implies that

$$
\mathrm{U}_{1}\left(\sigma_{-1} \diamond s\right) \geq \mathrm{U}_{1}\left(\sigma_{-1} \diamond t\right) .
$$

A contradiction.

\footnotetext{
${ }^{* *}$ We prepare the reader that this is the only property of the gadget game $\widehat{G}_{1}[2]$ that we shall use for the proof regarding the decision problems in Group $I V$. Thus, there could be used, as a gadget game, any win-lose game other than $\widehat{G}_{1}[2]$, such that there is, for each Nash equilibrium of it, a player receiving utility less than 1 .
} 
The claim now follows.

By Proposition 4.10, Lemma 7.4 immediately implies:

Corollary 7.5 Assume that $\phi$ is unsatisfiable. Then: (P.0) $\widetilde{\mathrm{G}} \| \widehat{\mathrm{G}}_{5}[k]$ has exactly $k$ Nash equilibria, and each is (P.1) Pareto-Optimal, (P.2) Strongly Pareto-Optimal, and (P.3) symmetric.

We continue to prove:

Lemma 7.6 Assume that $\phi$ is satisfiable. Then, (Q.1) \# $\phi \cdot(\# \phi+4)$ of the additional Nash equilibria for $\widetilde{\mathrm{G}} \| \widehat{\mathrm{G}}_{5}[k]$ are neither Pareto-Optimal nor Strongly Pareto-Optimal, (Q.2) \# $\phi$. $(\# \phi+1)$ of them are non-symmetric, and (Q.3) \#申 of them are symmetric.

Proof: Since $\phi$ is satisfiable, it follows, by Proposition 5.14, that for each satisfying assignment $\gamma$ of $\phi$, there is a Nash equilibrium $\boldsymbol{\sigma}$ for $\mathrm{G}$ such that for each player $i \in[2], \mathrm{U}_{i}(\boldsymbol{\sigma})=\frac{2}{n}<1$. Here are the additional Nash equilibria for $\widetilde{G}$ and their properties:

- The $(\phi)^{2}$ balanced mixtures of literal equilibria for G: By Lemma 6.3, $\boldsymbol{\sigma}^{4}$ is symmetric if and only if $\boldsymbol{\sigma}=\boldsymbol{\tau}$, while $\boldsymbol{\sigma}^{5}$ and $\boldsymbol{\sigma}^{6}$ are non-symmetric. Furthermore:

For each player $i \in[2], \mathrm{U}_{i}\left(\boldsymbol{\sigma}^{4}\right)=\frac{1}{n}<1$.

For each $\boldsymbol{\sigma} \in\left\{\boldsymbol{\sigma}^{5}, \boldsymbol{\sigma}^{6}\right\}: \sum_{i \in[2]} \mathrm{U}_{i}(\boldsymbol{\sigma})=\frac{2}{n}<2$.

- The $2 \cdot \# \phi$ balanced mixtures of a literal equilibrium and a gadget equilibrium for G: By Lemma 6.3, it follows that $\boldsymbol{\sigma}^{7}$ and $\boldsymbol{\sigma}^{8}$ are non-symmetric. Furthermore, by Lemma 6.4;

For each $\boldsymbol{\sigma} \in\left\{\boldsymbol{\sigma}^{5}, \boldsymbol{\sigma}^{6}\right\}$ : For each player $i \in[2]: \mathrm{U}_{i}\left(\boldsymbol{\sigma}^{7}\right)<\max \left\{\mathrm{U}_{i}(\boldsymbol{\sigma}), \mathrm{U}_{i}(\widehat{\boldsymbol{\sigma}})\right\}<$ 1.

Hence, there are $\# \phi$ additional Nash equilibria for $\widetilde{G}$ which are symmetric and $\# \phi \cdot(\# \phi+2)-$ $\# \phi=\# \phi \cdot(\# \phi+1)$ additional Nash equilibria for $\widetilde{\mathrm{G}}$ which are non-symmetric.

Consider now any additional Nash equilibrium $\boldsymbol{\sigma}$ for $\widetilde{\mathrm{G}}$. Since $\widetilde{\mathrm{G}} \| \widehat{\mathrm{G}}_{5}[k]$ is constructed from $\widetilde{G}$ by adding $T$ to the strategy set of each player, it suffices, in order to prove that $\boldsymbol{\sigma}$ is a Nash equilibrium for $\widetilde{G} \| \widehat{G}_{5}[k]$, to check that no player can improve her utility in $\boldsymbol{\sigma}$ by switching to a strategy in T. By Case (C.2) in the utility functions, it holds that $\mathrm{U}\left(\left\langle s_{1}, s_{2}\right\rangle\right)=\langle 0,0\rangle$ for $s_{1} \in \mathrm{L} \cup \mathrm{L}^{\prime}$ (resp., $s_{1} \in \mathrm{T}$ ) and $s_{2} \in \mathrm{T}$ (resp., $s_{2} \in \mathrm{L} \cup \mathrm{L}^{\prime}$ ). Hence, for each player $i \in[2]$, $\mathrm{U}_{i}\left(\boldsymbol{\sigma}_{-i} \diamond t\right)=0$ for each strategy $t \in \mathrm{T}$. Thus, $\boldsymbol{\sigma} \in \mathcal{N} \mathcal{E}\left(\widetilde{\mathrm{G}} \| \widehat{\mathrm{G}}_{5}[k]\right)$ with $\mathrm{U}_{i}(\boldsymbol{\sigma})<1$ for 
each player $i \in[2]$. Hence, $\widetilde{\mathrm{G}} \| \widehat{\mathrm{G}}_{5}[k]$ has $\# \phi$ symmetric Nash equilibria and $\# \phi \cdot(\# \phi+1)$ non-symmetric Nash equilibria. Properties (Q.2) and (Q.3) follow.

By Proposition 4.10, each player $i \in[2]$ has utility 1 in any Nash equilibrium for $\widehat{G}_{5}[k]$. Since $\mathcal{N} \mathcal{E}\left(\widehat{\mathrm{G}}_{5}[k]\right) \subseteq \mathcal{N} \mathcal{E}\left(\widetilde{\mathrm{G}} \| \widehat{\mathrm{G}}_{5}[k]\right)$, this implies that $\boldsymbol{\sigma}$ is neither Pareto-Otimal nor Strongly Pareto-Optimal. So there are $(\# \phi)^{2}+2 \cdot \# \phi+2 \cdot \# \phi \# \phi \cdot(\phi+4)$ additional Nash equilibria which are neither Pareto-Otimal nor Strongly Pareto-Optimal. Property (Q.1) follows.

Hence, using Lemma 7.6, we derive $\mathcal{N} \mathcal{P}$-hardness from the following table:

\begin{tabular}{|l|l|l|l|}
\hline \hline \multirow{2}{*}{ Decision Problem is $\mathcal{N} \mathcal{P}$-hard due to: } & \multicolumn{3}{|l|}{ Properties disentangling the satisfiability of $\phi:$} \\
\cline { 2 - 5 } & Unsat.: & \multicolumn{2}{l|}{ Sat.: } \\
\hline \hline$\exists k+1$ NASH & (P.0) & - & - \\
\hline$\exists \neg$ PARETO-OPTIMAL NASH & P.1) & $(\mathrm{Q} .1)$ & $\# \phi \cdot(\# \phi+4)$ \\
\hline$\exists \neg$ STRONGLY PARETO-OPTIMAL NASH & (P.2) & $(\mathrm{Q} .1)$ & $\# \phi \cdot(\# \phi+4)$ \\
\hline$\exists \neg$ SYMMETRIC NASH & (P.3) & $(\mathrm{Q} .2)$ & $\# \phi \cdot(\# \phi+1)$ \\
\hline \hline
\end{tabular}

Both formulas in the rightmost column can be solved for $\# \phi$; by the $\# \mathcal{P}$-hardness of computing $\# \phi$ [35], this yields the $\# \mathcal{P}$-hardness of the three counting problems. Furthermore, the formula $\# \phi \cdot(\# \phi+1)$ preserves the parity $\oplus \phi$; by the $\oplus \mathcal{P}$-hardness of computing $\oplus \phi$ [33], this yields the $\oplus \mathcal{P}$-hardness of the parity problems $\oplus \neg$ PARETO-OPTIMAL NASH and $\oplus \neg$ STRONGLY PARETO-OPTIMAL NASH.

By Corollary 7.5 and Lemma 7.6 (Condition (Q.3)), \# SYMMETRIC NASH $=k+\# \phi$. This formula can be solved for $\# \phi$; by the $\# \mathcal{P}$-hardness of computing $\# \phi$ [35], this yields the $\# \mathcal{P}$ hardness of \# SYMMETRIC NASH. Furthermore, this formula allows computing $\oplus \phi$ from $\oplus$ SYMMETRIC NASH; by the $\oplus \mathcal{P}$-hardness of computing $\oplus \phi[33$, this yields the $\oplus \mathcal{P}$-hardness of $\oplus$ SYMMETRIC NASH.

We now show:

Theorem 7.7 Fix a win-lose bimatrix game $\widehat{G}$ with the positive utility property. Then, restricted to symmetric win-lose bimatrix games, NASH-EQUIVALENCE $(\mathrm{GHR}(\widehat{\mathrm{G}}))$ is co-N $\mathcal{P}$ complete.

Proof: Here is a polynomial time algorithm to decide the non-satisfiability of an input formula $\phi$, with access to an oracle for NASH-EQUIVALENCE $(\mathrm{GHR}(\widehat{\mathrm{G}}))$ :

Construct the symmetric win-lose bimatrix games $\operatorname{GHR}(\widehat{\mathrm{G}})$ and $\widetilde{\mathrm{G}}=\mathrm{GHR}(\mathrm{G}(\widehat{\mathrm{G}}, \phi))$. Query the oracle for NASH-EQUIVALENCE $(\mathrm{GHR}(\widehat{\mathrm{G}}))$ on input $\widetilde{G}$ and return the answer of the oracle. 
To establish the correctness of the algorithm, we prove:

Lemma $7.8 \mathcal{N} \mathcal{E}(\mathrm{G}(\widehat{\mathrm{G}}, \phi))=\mathcal{N} \mathcal{E}(\widehat{\mathrm{G}})$ if and only if $\mathcal{N} \mathcal{E}(\mathrm{GHR}(\mathrm{G}(\widehat{\mathrm{G}}, \phi)))=\mathcal{N} \mathcal{E}(\mathrm{GHR}(\widehat{\mathrm{G}}))$.

Proof: Assume first that $\mathcal{N} \mathcal{E}(\mathrm{GHR}(\mathrm{G}(\widehat{\mathrm{G}}, \phi)))=\mathcal{N} \mathcal{E}(\mathrm{GHR}(\widehat{\mathrm{G}}))$. Since every Nash equilibrium for $\widehat{G}$ is a gadget equilibrium, the win-lose GHR-symmetrization implies that every Nash equilibrium for $\operatorname{GHR}(\widehat{G})$ is also a gadget equilibrium. Hence, the Nash-equivalence of $\mathrm{GHR}(\widehat{\mathrm{G}})$ and $\operatorname{GHR}(\mathrm{G}(\widehat{\mathrm{G}}, \phi))$ implies that every Nash equilibrium for $\operatorname{GHR}(\mathrm{G}(\widehat{\mathrm{G}}, \phi))$ is a gadget equilibrium. Hence, the win-lose GHR-symmetrization implies that every Nash equilibrium for $\mathrm{G}(\widehat{\mathrm{G}}, \phi)$ is a gadget equilibrium. By Propositions 5.13 and 5.14 , it follows that $\phi$ is unsatisfiable (since otherwise $\mathrm{G}(\widehat{\mathrm{G}}, \phi)$ has a literal equilibrium). Hence, Proposition 5.13 implies that $\mathcal{N E}(\mathrm{G}(\widehat{\mathrm{G}}, \phi))=\mathcal{N} \mathcal{E}(\widehat{\mathrm{G}})$.

Assume now that $\mathcal{N E}(\operatorname{GHR}(\mathrm{G}(\widehat{\mathrm{G}}, \phi))) \neq \mathcal{N} \mathcal{E}(\mathrm{GHR}(\widehat{\mathrm{G}}))$. Since $\mathcal{N} \mathcal{E}(\widehat{\mathrm{G}}) \subseteq \mathcal{N} \mathcal{E}(\mathrm{G}(\widehat{\mathrm{G}}, \phi))$, every gadget equilibrium is a Nash equilibrium for $\mathrm{G}(\widehat{\mathrm{G}}, \phi)$. Hence, Proposition 6.5 implies that $\mathcal{N E}(\mathrm{GHR}(\widehat{\mathrm{G}})) \subseteq \mathcal{N} \mathcal{E}(\mathrm{GHR}(\mathrm{G}(\widehat{\mathrm{G}}, \phi)))$. Hence, the assumption implies that $\operatorname{GHR}(\mathrm{G}(\widehat{\mathrm{G}}, \phi))$ has a literal equilibrium. By the GHR-symmetrization, this implies that $\mathrm{G}(\widehat{\mathrm{G}}, \phi)$ has a literal equilibrium. By Propositions 5.13 and 5.14, it follows that $\phi$ is satisfiable (since otherwise $\mathrm{G}(\widehat{\mathrm{G}}, \phi)$ has no literal equilibrium). Hence, Propositions 5.14 implies that $\mathcal{N E}(\mathrm{G}(\widehat{\mathrm{G}}, \phi)) \neq \mathcal{N} \mathcal{E}(\widehat{\mathrm{G}})$, and this completes the proof.

By Propositions 5.13 and 5.14, $\phi$ is unsatisfiable if and only if $\mathcal{N} \mathcal{E}(\mathrm{G}(\widehat{\mathrm{G}}, \phi))=\mathcal{N} \mathcal{E}(\widehat{\mathrm{G}})$. Hence, by Lemma 7.8, it follows that $\phi$ is unsatisfiable if and only if $\mathcal{N E}(\operatorname{GHR}(\mathrm{G}(\widehat{\mathrm{G}}, \phi)))=\mathcal{N} \mathcal{E}(\mathrm{GHR}(\widehat{\mathrm{G}}))$. Hence, NASH-EQUIVALENCE $(\operatorname{GHR}(\widehat{\mathrm{G}}))$ is co- $\mathcal{N} \mathcal{P}$-hard.

\subsection{Win-Lose Bimatrix Games}

We show:

Theorem 7.9 Restricted to win-lose bimatrix games, $\exists$ SYMMETRIC NASH is $\mathcal{N} \mathcal{P}$-complete.

Proof: Fix $\widehat{G}:=\widehat{G}_{4}$ (Section 4.4). Assume first that $\phi$ is unsatisfiable. Then, by Proposition 5.13, $\mathcal{N E}\left(\mathrm{G}\left(\widehat{\mathrm{G}}_{4}, \phi\right)\right)=\mathcal{N} \mathcal{E}\left(\widehat{\mathrm{G}}_{4}\right)$. Hence, by Proposition 4.9, $\mathrm{G}\left(\widehat{\mathrm{G}}_{4}, \phi\right)$ has no symmetric Nash equilibrium. Assume now that $\phi$ is satisfiable. Then, by Proposition 5.14 (Condition (4)), $\mathrm{G}\left(\widehat{\mathrm{G}}_{4}, \phi\right)$ has a symmetric Nash equilibrium. So $\mathrm{G}\left(\widehat{\mathrm{G}}_{4}, \phi\right)$ has a symmetric Nash equilibrium if and only if $\phi$ is satisfiable, and the $\mathcal{N} \mathcal{P}$-hardness of $\exists$ SYMMETRIC NASH follows.

We remark that the \#P-hardness of \# SYMMETRIC NASH for win-lose bimatrix games is already implied by the $\# \mathcal{P}$-hardness of \# SYMMETRIC NASH for symmetric win-lose bimatrix games (Theorem 7.1). 


\subsection{Win-Lose 3-Player Games}

We show:

Theorem 7.10 Restricted to win-lose 3-player games, $\exists$ RATIONAL NASH is $\mathcal{N} \mathcal{P}$-complete. Furthermore, \# RATIONAL NASH is \#P्P-complete and $\oplus$ RATIONAL NASH is $\oplus \mathcal{P}$-complete.

Proof: Fix $\widehat{G}:=\widehat{G}_{2}$ (Section 4.2). Assume first that $\phi$ is unsatisfiable. Then, by Proposition 5.13, $\mathcal{N E}\left(\mathrm{G}\left(\widehat{\mathrm{G}}_{2}, \phi\right)\right)=\mathcal{N} \mathcal{E}\left(\widehat{\mathrm{G}}_{2}\right)$. Hence, by Proposition 4.2, $\mathrm{G}\left(\widehat{\mathrm{G}}_{2}, \phi\right)$ has a single Nash equilibrium, which is irrational. Assume now that $\phi$ is satisfiable. Then, by Proposition 5.14 (Condition (4)), $\mathrm{G}\left(\widehat{\mathrm{G}}_{2}, \phi\right)$ has, for each satisfying assignment $\gamma$ of $\phi$, a rational Nash equilibrium $\boldsymbol{\sigma}=\boldsymbol{\sigma}(\gamma)$. Thus, $\mathrm{G}\left(\widehat{\mathrm{G}}_{2}, \phi\right)$ has a rational Nash equilibrium if and only if $\phi$ is satisfiable, and the $\mathcal{N} \mathcal{P}$-hardness of $\exists$ RATIONAL NASH follows. By Propositions 5.13 and 5.14, the number of rational Nash equilibria for $\mathrm{G}$ is $\# \phi$. Since computing $\# \phi$ is $\# \mathcal{P}$-hard [35], the $\# \mathcal{P}$-hardness of \# RATIONAL NASH follows. Since computing $\oplus \phi$ is $\oplus \mathcal{P}$-hard [33], $\oplus \mathcal{P}$-hardness of $\oplus$ RATIONAL NASH follows.

\section{Discussion and Open Problems}

We have established that symmetric win-lose bimatrix games are as complex as general bimatrix games with respect to a handful of decision problems about Nash equilibria, which either were previously studied in [4, 7, 12, 13, 20, 25, 26, 28] or are new (Theorems 7.1] and 7.7). Furthermore, deciding the existence of a symmetric Nash equilibrium for win-lose bimatrix games is as hard as for general bimatrix games (Theorem [7.9); deciding the existence of a rational Nash equilibrium for win-lose 3-player games is as hard as for general 3-player games (Theorem 7.10). Figure 4 provides a tabular summary of these $\mathcal{N} \mathcal{P}$-hardness results, which improve and extend previous corresponding $\mathcal{N} \mathcal{P}$-hardness results from [4, 17, 12, 13, 20, 28, there remain just two of those decision problems whose $\mathcal{N} \mathcal{P}$-hardness is still open; we conjecture:

Conjecture 8.1 $\exists$ PARETO-OPTIMAL NASH and $\exists$ STRONGLY PARETO-OPTIMAL NASH are $\mathcal{N} \mathcal{P}$-hard for symmetric win-lose bimatrix games.

Furthermore, the counting versions of these $\mathcal{N} \mathcal{P}$-hard problems (Theorems 7.1, 7.9 and 7.10) are all \#P-hard. Except for two of the parity versions of those $\mathcal{N} \mathcal{P}$-hard decision problems, namely $\oplus \neg$ UNIFORM NASH and $\oplus \neg$ SYMMETRIC NASH, their parity versions are all $\oplus \mathcal{P}$-hard. Of these two, recall that $\oplus \neg$ SYMMETRIC NASH is in $\mathcal{P}$; the $\oplus \mathcal{P}$-hardness of $\oplus \neg$ UNIFORM NASH remains open. We conjecture: 
Conjecture $8.2 \oplus \neg$ UNIFORM NASH is $\oplus \mathcal{P}$-hard.

The following decision problem inquires about the irrationality of Nash equilibria:

$\exists$ IRRATIONAL NASH

I.: A game $\mathrm{G}$.

Q.: Does $G$ have an irrational Nash equilibrium?

$\exists$ IRRATIONAL NASH is a trivial problem for non-degenerate bimatrix games $\mathcal{N} \mathcal{P}$-hard for general 3-player games [4, Theorem 4] $\$$

Conjecture 8.3 $\exists$ IRRATIONAL NASH is $\mathcal{N} \mathcal{P}$-hard for win-lose 3-player games.

Obtaining tight hardness results for the complexity of the decision problems about Nash equilibria (resp., symmetric Nash equilibria) in 3-player win-lose games (resp., symmetric 3-player win-lose games) remains a tantalizing open problem. In two very recent works [5, 6] extending [19], the present authors established tight $\exists \mathbb{R}$-hardness results for this complexity in general 3 -player games (resp., general symmetric 3-player games); $\exists \mathbb{R}$ is the complexity class capturing the Existential Theory of the Reals [34].

Open Problem 8.1 Determine whether or not the $\exists \mathbb{R}$-complete decision problems about Nash equilibria (resp., symmetric Nash equilibria) in 3-player games (resp., symmetric 3-player games) from [5, 6] remain $\exists \mathbb{R}$-hard when the corresponding games are restricted to win-lose.

\section{Acknowledgments:}

We would like to thank Burkhard Monien and Pino Persiano for some helpful comments.

\footnotetext{
${ }^{\dagger \dagger}$ For bimatrix games, the existence of an irrational Nash equilibrium is equivalent to the existence of infinitely many Nash equilibria. Non-degenerate bimatrix games have a finite number of Nash equilibria. Hence, they have no irrational Nash equilibrium.

$\ddagger$ The proof used an alternative approach, employing a reduction from the decision problem NASHREDUCTION [4, Section 2.3.2], which is not considered here.
} 


\begin{tabular}{|c|c|c|c|c|}
\hline \multirow[b]{2}{*}{ Decision problem: } & \multicolumn{4}{|c|}{$\mathcal{N P}$-hard for bimatrix games: } \\
\hline & G & $\mathrm{S}$ & $\mathbf{W L}$ & $\mathrm{S}+\mathrm{WL}$ \\
\hline$\exists k+1$ NASH (with $k \geq 1$ ) & $\Leftarrow$ & [13, 20] $(k=1) \& \Leftarrow$ & [12] $(k=1) \& \Leftarrow$ & $(7.1), \widehat{\mathrm{G}}_{1}[2] \& \widehat{\mathrm{G}}_{5}[k]$ \\
\hline$\exists$ NASH WITH LARGE UTILITIES & $\Leftarrow$ & {$[13,20] \& \Leftarrow$} & $\Leftarrow$ & (7.1), $\widehat{\mathrm{G}}_{1}[h]$ \\
\hline$\exists$ NASH WITH SMALL UTILITIES & $\Leftarrow$ & $\Leftarrow$ & $\Leftarrow$ & (7.1), $\widehat{\mathrm{G}}_{1}[1]$ \\
\hline$\exists$ NASH WITH LARGE TOTAL UTILITY & $\Leftarrow$ & $13, \& \Leftarrow$ & $\Leftarrow$ & (7.1), $\widehat{\mathrm{G}}_{1}[h]$ \\
\hline$\exists$ NASH WITH SMALL TOTAL UTILITY & $\Leftarrow$ & $\Leftarrow$ & $\Leftarrow$ & (7.1), $\widehat{\mathrm{G}}_{1}[1]$ \\
\hline$\exists$ NASH WITH LARGE SUPPORTS & $\Leftarrow$ & $13,20, \& \Leftarrow$ & $\Leftarrow$ & (7.1), $\widehat{\mathrm{G}}_{1}[1]$ \\
\hline$\exists$ NASH WITH SMALL SUPPORTS & $\Leftarrow$ & $20] \& \Leftarrow$ & $\Leftarrow$ & (7.1), $\widehat{\mathrm{G}}_{1}[h]$ \\
\hline$\exists$ NASH WITH RESTRICTED SUPPORTS & $\Leftarrow$ & {$[13,20, \& \Leftarrow$} & $\Leftarrow$ & (7.1), $\widehat{\mathrm{G}}_{1}[1]$ \\
\hline$\exists$ NASH WITH RESTRICTING SUPPORTS & $\Leftarrow$ & [13, 20] \& $\&$ & $\Leftarrow$ & (7.1), $\widehat{\mathrm{G}}_{1}[1]$ \\
\hline$\exists$ PARETO-OPTIMAL NASH & $\Leftarrow$ & 13 & $?$ & $?$ \\
\hline$\exists \neg$ PARETO-OPTIMAL NASH & $\Leftarrow$ & $\Leftarrow$ & $\Leftarrow$ & $(7.1), \widehat{\mathrm{G}}_{1}[2] \& \widehat{\mathrm{G}}_{5}[k]$ \\
\hline$\exists$ STRONGLY PARETO-OPTIMAL NASH & $\Leftarrow$ & 13 & $?$ & $?$ \\
\hline$\exists \neg$ STRONGLY PARETO-OPTIMAL NASH & $\Leftarrow$ & $\Leftarrow$ & $\Leftarrow$ & (7.1), $\widehat{\mathrm{G}}_{1}[2] \& \widehat{\mathrm{G}}_{5}[k]$ \\
\hline$\exists$ NASH WITH SMALL PROBABILITIES & $\Leftarrow$ & $\Leftarrow$ & $\Leftarrow$ & (7.1), $\widehat{\mathrm{G}}_{1}[1]$ \\
\hline$\exists$ UNIFORM NASH & $\Leftarrow$ & $\Leftarrow$ & [7] \& $\&$ & (7.1), $\widehat{\mathrm{G}}_{3}$ \\
\hline$\exists \neg$ UNIFORM NASH & $\Leftarrow$ & $\Leftarrow$ & $\Leftarrow$ & $(7.1), \widehat{G}_{1}[1]$ \\
\hline$\exists$ SYMMETRIC NASH & $\Leftarrow$ & $\perp$ & $(7.9), \widehat{\mathrm{G}}_{4}$ & $\perp$ \\
\hline$\exists \neg$ SYMMETRIC NASH & $\Leftarrow$ & $28, \& \Leftarrow$ & $\Leftarrow$ & $(7.1), \widehat{\mathrm{G}}_{1}[2] \& \widehat{\mathrm{G}}_{5}[k]$ \\
\hline "NASH-EQUIVALENCE $(\widehat{\mathrm{G}})$ & [4] & $\Leftarrow$ & $\bar{E} \Leftarrow$ & (7.7), $\widehat{\text { G }}$ \\
\hline NASH-EQUIVALENCE & 4 & $\Downarrow$ & $\Downarrow$ & $\Downarrow$ \\
\hline
\end{tabular}

\begin{tabular}{||l|c|c||}
\hline & \multicolumn{2}{|c||}{$\mathcal{N} \mathcal{P}$-hard for 3-player games } \\
\hline Decision problem: & $\mathbf{G}$ & $\mathbf{W L}$ \\
\hline \hline$\exists$ RATIONAL NASH & {$[4]$} & (7.10), $\widehat{\mathrm{G}}_{2}$ \\
\hline$\exists$ IRRATIONAL NASH & {$[4]$} & $?$ \\
\hline \hline
\end{tabular}

Figure 4: Summary of the $\mathcal{N} \mathcal{P}$-hardness results for decision problems about Nash equilibria, and comparison to previous related work in [4, 7, 12, 13, 20, 28, Columns correspond to classes of bimatrix games; specifically, the symbols $\mathbf{G}, \mathbf{S}, \mathbf{W L}$ and $\mathbf{S}+\mathbf{W L}$ denote general, symmetric, win-lose and simultaneously symmetric and win-lose bimatrix games, respectively. The rightmost column gives the number of the corresponding theorem (in parenthesis), followed by the gadget used in the $\mathcal{N} \mathcal{P}$-hardness proof. The symbol "?" indicates that the $\mathcal{N} \mathcal{P}$-hardness of the decision problem remains open for the corresponding class. The symbol $\perp$ indicates that the decision problem is trivial for the corresponding class. An arrow $\Leftarrow$ in some entry indicates that $\mathcal{N} \mathcal{P}$-hardness follows directly from a corresponding $\mathcal{N} \mathcal{P}$-hardness result for some entry in the same row and to its right. An arrow $\Downarrow$ in some entry indicates that $\mathcal{N} \mathcal{P}$-hardness follows directly from the $\mathcal{N} \mathcal{P}$-hardness result for the entry in the same column and just above it. The first table applies to bimatrix games, while the second table applies to 3-player games. 


\section{References}

[1] T. Abbott, D. Kane and P. Valiant, "On the Complexity of Two-Player Win-Lose Games," Proceedings of the 46th Annual IEEE Symposium on Foundations of Computer Sciences, pp. 113-122, October 2005.

[2] L. Addario-Berry, N. Olver and A. Vetta, "A Polynomial Time Algorithm for Finding Nash Equilibria in Planar Win-Lose Games," Journal of Graph Algorithms and Applications, Vol. 11, No. 1, pp. 309-319, 2007.

[3] V. Bilò and A. Fanelli, "Computing Exact and Approximate Nash Equilibria in 2-Player Games," Proceedings of the 6th International Conference on Algorithmic Aspects in Information and Management, pp. 58-69, Vol. 6124, Lecture Notes in Computer Science, Springer-Verlag, June 2010.

[4] V. Bilò and M. Mavronicolas, "Complexity of Rational and Irrational Nash Equilibria," Theory of Computing Systems, Vol. 54, No. 3, pp. 491-527, April 2014.

[5] V. Bilò and M. Mavronicolas, "A Catalog of $\exists \mathbb{R}$-Complete Decision Problems about Nash Equilibria in Multi-Player Games," Proceedings of the 33rd International Symposium on Theoretical Aspects of Computer Science, Article No. 17, pp. 17:1-17:13, Vol. 47, LIPIcs, Schloss Dagstuhl - Leibniz Zentrum fuer Informatik, February 2016.

[6] V. Bilò and M. Mavronicolas, " $\exists \mathbb{R}$-Complete Decision Problems about Symmetric Nash Equilibria in Symmetric Multi-Player Games," Proceedings of the 34th International Symposium on Theoretical Aspects of Computer Science, Article No. 13, pp. 13:1-13:13, Vol. 66, LIPIcs, Schloss Dagstuhl - Leibniz Zentrum fuer Informatik, March 2017.

[7] V. Bonifaci, U. Di Orio and L. Laura, "The Complexity of Uniform Nash Equilibria and Related Regular Subgraph Problems," Theoretical Computer Science, Vol. 401, Nos. 1-3, pp. 144-152, July 2008.

[8] G. W. Brown and J. von Neumann, "Solutions of Games by Differential Equations," Contributions to the Theory of Games, Annals of Mathematics Studies, No. 24, pp. 73-79, Princeton University Press, 1950.

[9] X. Chen, X. Deng and S.-H. Teng, "Settling the Complexity of Computing Two-Player Nash Equilibria," Journal of the ACM, Vol. 56, No. 3, 2009.

[10] X. Chen, S.-H. Teng and P. Valiant, "The Approximation Complexity of Win-Lose Games," Proceedings of the 18th Annual ACM-SIAM Symposium on Discrete Algorithms, pp. 159168, January 2007. 
[11] B. Codenotti, M. Leoncini and G. Resta, "Efficient Computation of Nash Equilibria for Very Sparse Win-Lose Bimatrix Games," Proceedings of the 14th European Symposium on Algorithms, pp. 232-243, Vol. 4168, Lecture Notes in Computer Science, Springer-Verlag, September 2006.

[12] B. Codenotti and D. Štefankovič, "On the Computational Complexity of Nash Equilibria for $(0,1)$ Bimatrix Games," Information Processing Letters, Vol. 94, No. 3, pp. 145-150, May 2005.

[13] V. Conitzer and T. Sandholm, "New Complexity Results about Nash Equilibria," Games and Economic Behavior, Vol. 63, No. 2, pp. 621-641, July 2008.

[14] C. Daskalakis, P. W. Goldberg and C. H. Papadimitriou, "The Complexity of Computing a Nash Equilibrium," SIAM Journal on Computing, Vol. 39, No. 1, pp. 195-259, 2009.

[15] S. Datta and N. Krishnamurthy, "Some Tractable Win-Lose Games," Proceedings of the 8th Annual Conference on Theory and Applications of Models of Computation, pp. 365-376, Vol. 6648, Lecture Notes in Computer Science, Springer-Verlag, May 2011.

[16] K. Etessami and M. Yannakakis, "On the Complexity of Nash Equilibria and Other Fixed Points," SIAM Journal on Computing, Vol. 39, No. 6, pp. 2531-2597, 2010.

[17] D. Gale, H. W. Kuhn and A. W. Tucker, "On Symmetric Games," Contributions to the Theory of Games, Annals of Mathematics Studies, Vol. 24, Princeton University Press, pp. 81-87, 1950.

[18] M. R. Garey and D. S. Johnson, Computers and Intractability - A Guide to the Theory of $\mathcal{N} \mathcal{P}$-Completeness, W. H. Freeman \& Co., 1979.

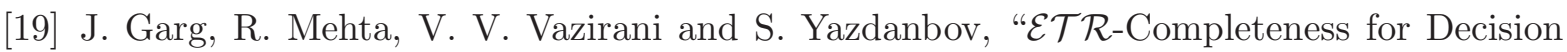
Versions of Multi-Player (Symmetric) Nash Equilibria," Proceedings of the 42nd International Colloquium on Automata, Languages and Programming, pp. 554-566, Vol. 9134, Lecture Notes in Computer Science, Springer-Verlag, July 2015.

[20] I. Gilboa and E. Zemel, "Nash and Correlated Equilibria: Some Complexity Considerations," Games and Economic Behavior, Vol. 1, No. 1, pp. 80-93, March 1989.

[21] J. H. Griesmer, A. J. Hoffman and A. Robinson, "On Symmetric Bimatrix Games," IBM Research Paper RC-959, IBM Corp., T. J. Watson Research Center, 1963.

[22] M. J. M. Jansen, J. A. M. Potters and S. H. Tijs, "Symmetrizations of Two-Person Games," Methods of Operations Research, Vol. 54, pp. 385-402, 1986. 
[23] A. P. Jurg, M. J. M. Jansen, J. A. M. Potters and S. H. Tijs, "A Symmetrization for Finite Two-Person Games," Methods and Models of Operations Research, Vol. 36, pp. 111-123, 1992.

[24] M. Mavronicolas and B. Monien, "The Complexity of Equilibria for Risk-Modeling Valuations," Theoretical Computer Science, Vol. 634, pp. 67-96, June 2016.

[25] A. McLennan and R. Tourky, "Simple Complexity from Imitation Games," Games and Economic Behavior, Vol. 68, No. 2, pp. 683-688, March 2010.

[26] A. McLennan and R. Tourky, "Imitation Games and Computation," Games and Economic Behavior, Vol. 70, No. 1, pp. 4-11, September 2010.

[27] N. Megiddo and C. H. Papadimitriou, "On Total Functions, Existence Theorems and Computational Complexity," Theoretical Computer Science, Vol. 81, pp. 317-324, April 1991.

[28] R. Mehta, V. V. Vazirani and S. Yazdanbod, "Settling Some Open Problems on 2-Player Symmetric Nash Equilibria," Proceedings of the 8th International Symposium on Algorithmic Game Theory, pp. 272-284, Vol. 9347, Lecture Notes in Computer Science, SpringerVerlag, October 2015.

[29] J. F. Nash, "Equilibrium Points in $n$-Person Games," Proceedings of the National Academy of Sciences of the United States of America, Vol. 36, pp. 48-49, 1950.

[30] J. F. Nash, "Non-Cooperative Games," Annals of Mathematics, Vol. 54, No. 2, pp. 286-295, 1951.

[31] C. H. Papadimitriou, "On the Complexity of the Parity Argument and Other Inefficient Proofs of Existence," Journal of Computer and System Sciences, Vol. 48, No. 3, pp. 498532, August 1994.

[32] C. H. Papadimitriou, "The Complexity of Finding Nash Equilibria," Chapter 2 in N. Nisan, T. Roughgarden, É. Tardos and V. V. Vazirani eds., Algorithmic Game Theory, pp. 29-50, Cambridge University Press, 2007.

[33] C. H. Papadimitriou and S. Zachos, "Two Remarks on the Power of Counting," Proceedings of Theoretical Computer Science, 6th GI-Conference, pp. 269-276, Vol. 145, Lecture Notes in Computer Science, Springer-Verlag, January 1983.

[34] M. Schaefer and D. Štefankovič, "Fixed Points, Nash Equilibria and the Existential Theory of the Reals," Theory of Computing Systems, Vol. 60, No. 2, pp. 172-193, February 2017. 
[35] L. G. Valiant, "The Complexity of Computing the Permanent," Theoretical Computer Science, Vol. 8, No. 2, pp. 189-201, 1979.

[36] L. G. Valiant, "Completeness for Parity Problems," Proceedings of the 11th Annual International Conference on Computing and Combinatorics, pp. 1-8, Vol. 3595, Lecture Notes in Computer Science, Springer-Verlag, August 2005. 